Portland State University

PDXScholar

Summer 8-16-2017

\title{
Expanding Metabolic Diversity of Two Archaeal Phyla: Nanoarchaeota and Korarchaeota
}

John Forad Kelley

Portland State University

Follow this and additional works at: https://pdxscholar.library.pdx.edu/open_access_etds

Part of the Biology Commons

Let us know how access to this document benefits you.

Recommended Citation

Kelley, John Forad, "Expanding Metabolic Diversity of Two Archaeal Phyla: Nanoarchaeota and Korarchaeota" (2017). Dissertations and Theses. Paper 3835.

https://doi.org/10.15760/etd.5729

This Thesis is brought to you for free and open access. It has been accepted for inclusion in Dissertations and Theses by an authorized administrator of PDXScholar. Please contact us if we can make this document more accessible: pdxscholar@pdx.edu. 
Expanding Metabolic Diversity of Two Archaeal Phyla:

Nanoarchaeota and Korarchaeota

by

John Forad Kelley

A thesis submitted in partial fulfillment of the requirements for the degree of

Master of Science

in

Biology

Thesis Committee:

Anna-Louise Reysenbach, Chair

Michael Bartlett

Mircea Podar

Rahul Raghavan

Portland State University

2017 


\begin{abstract}
Culture independent studies have revealed a greater diversity of Archaea than the two kingdoms initially defined by Carl Woese. Culturing species from the newly discovered archaeal lineages, as with the majority of microbes, has been difficult. To overcome the culturing dilemma, metagenomics is being used to reconstruct environmental genomes. Two metagenomic studies are presented in this thesis, with the aim of recovering newly proposed archaeal genomes from the Nanoarchaeota and Korarchaeota.

In the first study, a sediment sample, NZ13, was collected from a terrestrial New Zealand hot spring. Along with the sediment sample, two complex enrichments were sequenced on an Illumina MiSeq platform. Assembly and differential binning recovered two nearly complete genomes of a nanoarchaeote and a korarchaeote. The NZ13 nanoarchaeote is similar to other terrestrial nanoarchaeotes, which lack an ATP synthase and encode genes for glycolysis/gluconeogenesis and archaella. One notable difference is the NZ13 nanoarchaeote contains CRISPR genes, which are absent in other terrestrial nanoarchaeotes, although present in a marine nanoarchaeote, Nanoarchaeum equitans. The NZ13 korarchaeote mirrors Candidatus Korarchaeum cryptofilum, lacking genes for de novo synthesis of purines and several cofactors, while containing an abundance of peptide transporters and amino acid fermentation pathways.

The second study focused on sulfide samples collected from deep-sea hydrothermal vent fields in southwestern Pacific Ocean along the Eastern Lau Spreading Center. Ten sulfide samples were sequenced on an Illumina HiSeq platform. Small
\end{abstract}


subunit ribosomal RNA genes were extracted from the metagenome reads and aligned against the SILVA Ref NR 99123 database. The preliminary results identified which samples could be prioritized for genome reconstruction of uncultured bacterial and archaeal lineages. Three uncultured bacteria, candidate division SR1, Gracilibacteria (GN02) and Parcubacteria (OD1) were identified in several samples. Many uncultured deep-sea hydrothermal archaeal lineages were identified in all samples. In particular, korarchaeotal sequences were in high relative archaeal abundances in two samples, ABE-1 and Vai Lili-2, while few nanoarchaeotal reads were classified. 


\section{Acknowledgements}

I would like to thank my advisor, Anna-Louise Reysenbach, for being a continuing inspiration on my journey to become a research scientist. I would also like to thank my committee members Michael Bartlett, Mircea Podar and Rahul Raghavan for their support through my project. I would also like to acknowledge the past and current members of both the Reysenbach and Raghavan lab, their help made my research possible. In particular, I would like to thank Yitai Liu and Kristen Brileya for their culturing work, which provided my sequence data. Rick Davis, Todd Smith and Jennifer Meneghin were instrumental in teaching me how to process the sequence data. The Hedlund lab graciously provided korarchaeotal sequence data. In addition, a special thanks to our collaborators at GNS Science, especially Matt Stott, for providing access to the geothermal pools, with the permission of the local Māori tribe, the Ngāti Rangiteaorere. Also, thanks to the crew of the R/V Roger Revelle and the ROV Jason II, my time aboard the ship was truly amazing. Lastly, I would like to thank my wife, Abby, for her continued support during these past couple years. Funding for this research was supported by National Science Foundation grants DEB-1134877 and OCE-1235432 to Anna-Louise Reysenbach. 
Table of Contents

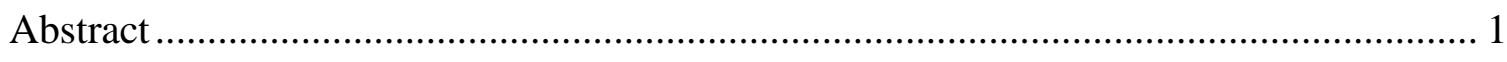

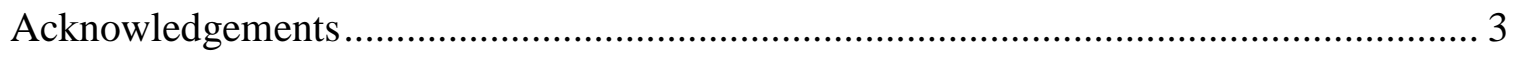

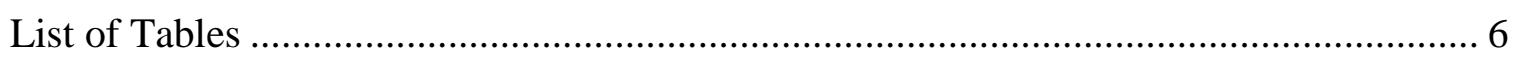

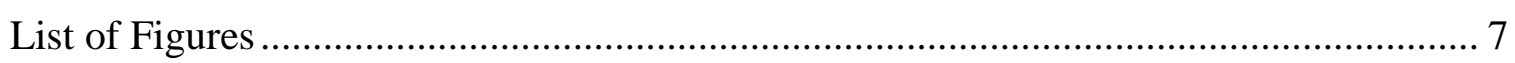

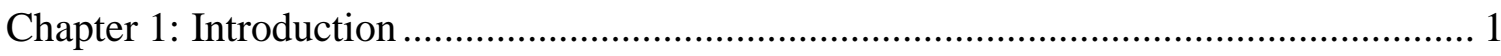

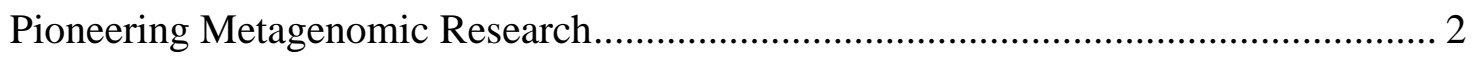

Genome Recovery from Environmental Samples........................................................ 3

Archaeal Expansion: Korarchaeota and Nanoarchaeota................................................. 4

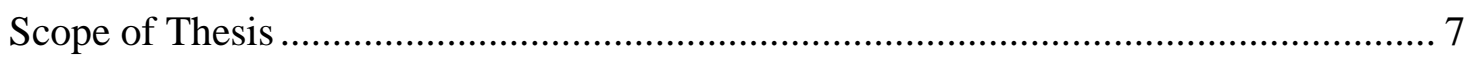

Chapter 2: Genomic analysis of New Zealand nanoarchaeotal and korarchaeotal genomes reveals a conserved metabolism among terrestrial lineages .............................................. 8

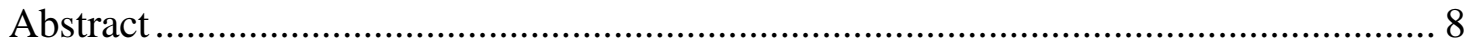

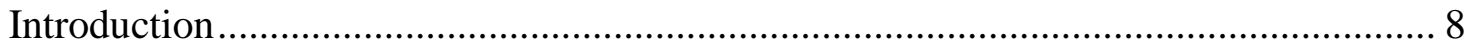

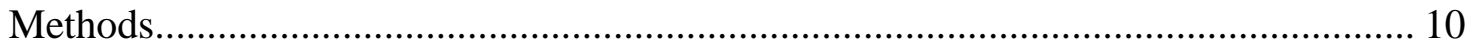

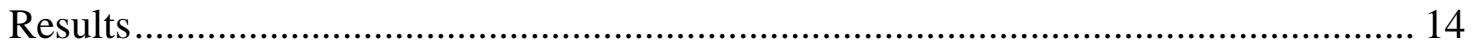

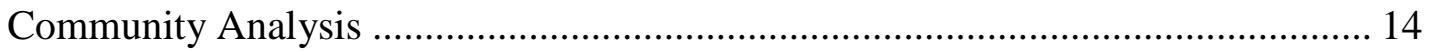

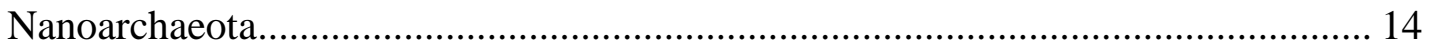

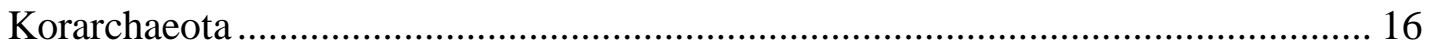

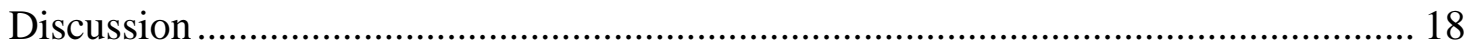

Chapter 3: Microbial Community Composition of Deep-sea Hydrothermal Vents from the Eastern Lau Spreading Center ………………………................................................ 37

Abstract troduction . 
Appendix A: Supplemental information from the nanoarchaeotal and korarchaeotal

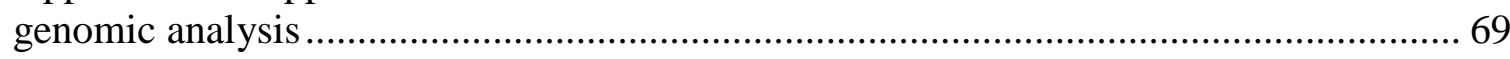

Appendix B: Sulfide sample details from the Eastern Lau Spreading Center................... 96 


\section{List of Tables}

Table 1. Summary of raw data from the three metagenomes and co-assembly. NZ13 is the environmental sample 23

Table 2. Comparison of general features of nanoarchaeote genomes ........................... 25 Table 3. Overview of nanoarchaeotal similarities and differences: shared split proteins,

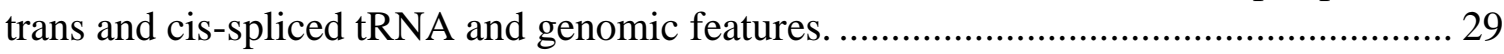

Table 4. General features table comparing three korarchaeotal genomes ....................... 32

Table 5. Raw sequence data, trimmed reads and SSU rRNA read counts by site ........... 46

Table 6. Summary of epsilonproteobacterial abundances. ........................................ 47 


\section{List of Figures}

Figure 1. Relative abundance of SSU rRNA trimmed read counts from NZ13, NZ13en-R

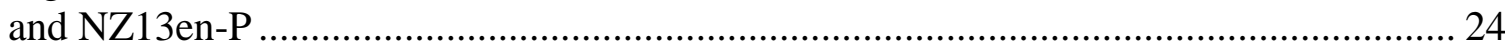

Figure 2. NZ13 nanoarchaeotal genome plot............................................................... 26

Figure 3. Relative abundance of arCOG categories from ORFs in nanoarchaeotal genomes

Figure 4. NZ13 nanoarchaeotal BLASTp synteny plots. A. NZ13 nanoarchaeotal protein to protein comparison against Nanopusillus acidilobi B. NZ13 nanoarchaeotal protein to protein comparison against Nanoarchaeum equitans.

Figure 5. Metabolic chart of NZ13 Nanoarchaeota. ………........................................... 30

Figure 6. Archaellum gene locations and orientation in the terrestrial Nanoarchaeota genomes: NZ13 Nanoarchaeota, N. acidilobi and Nst1. Sulfolobus and Pyrococcus are shown to illustrate a typical archaellum operon structure. ……....................................... 31

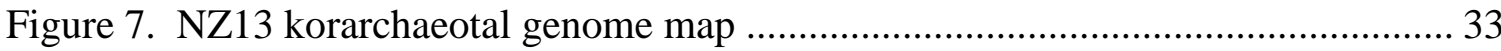

Figure 8. NZ13 korarchaeotal BLASTp synteny plots ………………………………....... 34

Figure 9. NZ13 korarchaeotal metabolism chart from the draft genome. ........................ 35

Figure 10. Relative abundance of arCOG categories from ORFs in korarchaeotal

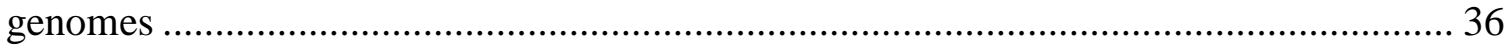

Figure 11. Hydrothermal vent fields along ELSC and VFR......................................... 45 Figure 12. Relative abundance of bacterial families from sulfide samples taken along the ELSC and VFR 49

Figure 13. Relative abundance of archaeal families from sulfide samples taken along the ELSC and VFR 50 


\section{Chapter 1: Introduction}

Microbes account for most of our planet's biological diversity, yet more than a century of cultivation approaches have isolated only a small fraction of the microbial life (Torsvik et al. 1990; Ward et al. 1990; Giovannoni et al. 1990). Through microscopy, early microbiologists knew the natural environment was more complex than their cultures. The advent of DNA sequencing unveiled the true complexities of the microscopic world, through identification of microbes using cultivation independent techniques (Barns et al. 1994; Barns et al. 1996).

The small subunit ribosomal RNA (SSU rRNA) is conserved evolutionarily and was first exploited by Carl Woese to redefine the Tree of Life (Fox et al. 1977). Woese's work resulted in the reclassification of prokaryotes from a single kingdom into two domains: Bacteria and Archaea (Fox et al. 1977; Woese et al. 1990). For the first time, a phylogenetic tree could be drawn from DNA sequences and subsequently, the SSU rRNA gene became a widespread method for prokaryotic identification. The SSU rRNA redefined evolutionary relationships, but the bulk of the uncultivated microbial genomic information was out of reach to researchers prior to whole genome sequencing. From Woese's rRNA-based reclassification, nearly two decades passed until the first bacterial genome, Haemophilus influenza, was fully sequenced from an isolate (Fleischmann et al., 1995). Soon thereafter, the requirement for lab cultures was bypassed as a precursor for genomic sequencing (Stein et al. 1996). Sequencing of all DNA isolated from environmental samples was demonstrated and termed metagenomics (Handelsman et al. 1998). 


\section{Pioneering Metagenomic Research}

The first metagenomic studies cloned environmental DNA fragments to construct sequence libraries. Environmental DNA was extracted, digested into large fragments and randomly inserted into vectors. The recombinant DNA was then fragmented again for shotgun sequencing. Then overlapping sequences were reassembled to generate contiguous strands of DNA known as contigs (Figure 14). An initial study used fosmid vectors which would accept DNA inserts up to 40 kilobases (kb) (Stein et al. 1996). Subsequent studies used bacterial artificial chromosome (BAC) vectors with DNA inserts of up to $750 \mathrm{~kb}$ (Stone et al. 1996; Handelsman et al. 1998).

A metagenomic study by Béjà et al., 2000 of oceanic surface waters revealed the existence of rhodopsin in a bacterial genome. A $130 \mathrm{~kb}$ BAC vector encoded for a rRNA operon belonging to SAR86, an uncultivated bacterium from the $\gamma$-Proteobacteria (Béjà et al. 2000). The SAR86 genomic fragment contained a rhodopsin gene, a light dependent proton pump. Prior to this study, the rhodopsin genes were only known in Archaea and Eukarya. To investigate whether the bacterial rhodopsin gene was functional, it was cloned and expressed in Escherichia coli. In the presence of retinal, cells expressing rhodopsin developed pigmentation and showed a peak absorbance of 520 $\mathrm{nm}$, a wavelength of light that travels far below the ocean's surface. This discovery explained why the SAR86 clade was localized to the marine euphotic zone, the region where sunlight penetrates. The small genomic fragment contained important information about microbial physiology of SAR86, but was still only a snapshot of the uncultured genome. 


\section{Genome Recovery from Environmental Samples}

Some of the first nearly complete environmental genomes were reconstructed from a metagenome of a subsurface acid mine drainage (AMD) microbial community (Tyson et al. 2004). The AMD was selected for the low diversity, self-sustaining microbial community that received little to no external nutrients. With a low diversity microbial community, enough DNA fragments were sequenced, assembled into contigs and enabled genomic reconstruction. Contigs were grouped into single genomic bins using GC content and sequencing coverage. The results were two nearly complete genomes and three partial genomes, a near full recovery of the genomic DNA from the microbial community (Tyson et al., 2004).

The AMD microbial community required the machinery for assimilating carbon and nitrogen. As expected, several recovered genomes contained carbon fixation pathways that utilize the Calvin-Benson-Bassham cycle or reductive acetyl coenzyme A pathway. The primary nitrogen fixer was expected to be the dominant bacterial species, Leptospirillum group II. However, the nitrogen assimilation genes were located on a binned genome belonging to an uncultivated, relatively low abundance community member, Leptospirillum group III. Subsequently, the results led to the isolation of Leptospirillum ferrodiazotrophum, providing a cultured representative for a lineage in the Nitrospirae (Tyson et al. 2005).

The example above provided evidence that genome directed culturing was possible. However, there have only been a few examples where this method led to the successful isolation of novel microbes (e.g. Könneke et al. 2005; Bomar et al. 2011; Wurch et al. 2016). Instead, microbial ecology is still reliant on metagenomics or other 
meta-omics to explore microbial diversity.

\section{Archaeal Diversity Expansion: Korarchaeota and Nanoarchaeota}

Analysis of SSU rRNA genes from cultured isolates supported the initial division of the Archaea, creating the Crenarchaeota and Euryarchaeota kingdoms (Woese et al. 1990). The Euryarchaeota contained methanogenic, thermophilic and halophilic species, while the Crenarchaeota mostly comprised of thermophiles. Environmental SSU rRNA gene surveys exposed a new archaeal lineage, the Korarchaeota, that was grouped at the phyla level (Barns et al. 1994). The korarchaeotal SSU rRNA genes are endemic to terrestrial and marine thermophilic hot springs, ranging from mildly acidic to neutral (Barns et al. 1994; Auchtung et al. 2006; Miller-Coleman et al. 2012). Phylogenetic analysis indicated that the Korarchaeota cluster into five groups separated by geographic location (Auchtung et al. 2006). Additionally, there has been difficulty in obtaining a member from the phylum in culture (Elkins et al. 2008).

The first korarchaeotal genome was obtained from an enrichment culture of a sample from Obsidian Pool, Yellowstone National Park (YNP) (Elkins et al. 2008). Korarchaeotal specific SSU rRNA probes showed long, thin $(>0.2 \mu \mathrm{m})$ filaments ranging in length from $15 \mu \mathrm{m}$ to $100 \mu \mathrm{m}$ (Elkins et al. 2008). From the genome, $\mathrm{Ca}$. K. cryptofilum utilizes peptide fermentation pathways for carbon and energy (Elkins et al. 2008). Pathways for the biosynthesis of purines and several cofactors are absent, suggesting a dependency on another organism or nutrient scavenging. Protein homology analysis found many crenarchaeotal and euryarchaeotal specific genes which supports a deep-branching archaeal lineage. 
Another proposed ancestral archaeal lineage, the Nanoarchaeota, was previously not detected using environmental SSU rRNA gene surveys. Several base pair changes in the highly conserved regions of the SSU rRNA gene prevented universal primers from binding (Huber et al. 2002). The Nanoarchaeota were discovered while isolating the hyperthermophilic crenarchaeote, Ignicoccus hospitalis, from a shallow hydrothermal vent off the coast of Iceland (Huber et al. 2002). Many tiny cocci, less than $400 \mathrm{~nm}$ in diameter, were attached to its outer membrane and could only be grown in a coculture with I. hospitalis. The nanoarchaeotal cells were physically isolated for sequencing, producing the genome of Nanoarchaeum equitans (Waters et al. 2003).

The genome of $N$. equitans is greatly reduced, consisting of only $490 \mathrm{kbp}$ and has many split proteins and tRNAs (Waters et al. 2003; Randau et al. 2005). The genome lacks synthesis pathways for lipids, cofactors, amino acids and nucleotides. Therefore, $N$. equitans is dependent on its host for most of its nutrients, but has retained molecular machinery genes for genetic processing, repair and replication.

Two nanoarchaeotal genomes have recently been sequenced from YNP. Nanopusillus acidilobi genome has been fully sequenced (Wurch et al. 2016) and Nst1 consists of 7 contigs (Podar et al. 2013). N. acidilobi and Nst1 are characterized by three important differences from N. equitans: an absence of an ATP synthase complex, the presence of archaellum genes and a near complete gluconeogenesis/glycolysis pathway (Podar et al. 2013; Wurch et al. 2016). All three nanoarchaeotal genomes share in common a reduced genome, the lack of biosynthesis pathways and the dependency on a host for growth (Waters et al. 2003; Podar et al. 2013; Wurch et al. 2016). 
All three nanoarchaeotal hosts are from different crenarchaeotal orders, Desulfurococcales, Acidilobales and Sulfolobales (Huber et al. 2002; Podar et al. 2013; Wurch et al. 2016). Furthermore, N. equitans host is an autotroph (Huber et al. 2000), likewise Nst1 proposed host is an autotroph (Podar et al. 2013), while N. acidilobi host is a heterotroph (Wurch et al. 2016). In the nanoarchaeotal cocultures, $N$. equitans reduced its host ability to reproduce (Jahn et al. 2008), while N. acidilobi did not have an apparent negative effect on its host growth (Wurch et al. 2016). Further nanoarchaeotal-host characterization, either through cultivation or single cell genomics, should reveal the diversity of hosts utilized by different nanoarchaeotal species.

Originally the Korarchaeota and Nanoarchaeota were proposed as sister phyla to the Crenarchaeota and Euryarchaeota (Barns et al. 1996; Huber et al. 2002). Since then, metagenomics has expanded the archaeal domain, with proposals that the Korarchaeota and Nanoarchaeota are members of the TACK and DPANN superphyla, respectively (Rinke et al. 2013). Recently, the SSU rRNA gene has been used in conjunction with full-length ribosomal protein sequences yielding higher-resolution phylogenetic trees, inclusive of uncultivated lineages. Still, the basis for these new superphyla has been controversial (Hug et al. 2016). Many proposed phyla like the Korarchaeota and Nanoarchaeota have limited genomic representation. Nonetheless, the number of archaeal phyla continues to expand and for a more robust analysis, additional genomic data, from metagenomics and cultivation, needs to be gathered from diverse locations before a phylogenetic consensus can be reached. 


\section{Scope of Thesis}

The main goal of this study was to expand understanding of microbial diversity, with a specific focus on Korarchaeota and Nanoarchaeota from high temperature environments using metagenomic sequencing. In Chapter 2, I used differential binning to recover and refine a nanoarchaeote and a korarchaeote genome from a New Zealand hot spring. I annotated both genomes and compared their physiologies to available nanoarchaeotal and korarchaeotal genomes. In Chapter 3, I performed the initial metagenomic processing for ten hydrothermal vent sulfide samples from the Eastern Lau Spreading Center in the southwestern Pacific Ocean. I extracted the SSU rRNA reads from each metagenome and aligned the reads against a SSU rRNA reference database, creating a community profile for each sample. I also identified which samples would be best for genome recovery of uncultivated bacterial and archaeal lineages from the deepsea hydrothermal vent samples. 


\title{
Chapter 2: Genomic analysis of New Zealand nanoarchaeotal and korarchaeotal genomes reveals a conserved metabolism among terrestrial lineages
}

\author{
Abstract \\ The Nanoarchaeota and Korarchaeota represent two poorly characterized, deep- \\ branching archaeal phyla, with few sequenced genomes. Both lineages have been \\ detected globally in terrestrial and marine high temperature environments, but have been \\ difficult to cultivate in the laboratory. All cultivated Nanoarchaeota form \\ ectosymbiotic/parasitic associations with their crenarchaeotal hosts, while no \\ Korarchaeota have been grown in pure culture. In order to further characterize and \\ obtain additional insights into their potential physiologies, metagenomes of \\ environmental and enrichment samples containing nanoarchaeotes and korarchaeotes \\ were analyzed from a New Zealand terrestrial hot spring (NZ13). The NZ13 \\ nanoarchaeotal genome is similar to other terrestrial nanoarchaeotes from Yellowstone \\ National Park with similar metabolism, archaellum genes and lack of an ATP synthase. \\ The NZ13 korarchaeote mirrors $C a$. K. cryptofilum, which lack genes for de novo \\ synthesis of purines and several cofactors, while containing an abundance of peptide \\ transporters and amino acid fermentation pathways.
}

\section{Introduction}

Since their discovery, the Nanoarchaeota and Korarchaeota have been detected globally in terrestrial and marine high temperature environments using small subunit (SSU) rRNA gene surveys (Barns et al. 1994; Huber et al. 2002; Auchtung et al. 2006; Casanueva et al. 2008; Miller-Coleman et al. 2012). Their phylogenetic placement within the Archaea has remained ambiguous and has become further muddled as more 
archaeal phyla continue to be discovered (Brochier-Armanet et al. 2008; Rinke et al. 2013; Spang et al. 2015; Baker et al. 2016). SSU rRNA alignments show phylogenetic diversity and geographical clustering among each respective phylum, especially between terrestrial and marine lineages.

Currently, the Nanoarchaeota are represented by one marine and one terrestrial isolate, both in a coculture with their respective obligate crenarchaeotal host (Waters et al. 2003; Wurch et al. 2016). The first isolate to be sequenced was Nanoarchaeum equitans. N. equitans was obtained from an Icelandic hydrothermal vent and attached to its crenarchaeotal host, the autotrophic Ignicoccus hospitalis (Huber et al. 2002). The highly reduced $N$. equitans genome is $490 \mathrm{kbp}$ (kilobase pair), with a large portion of the genome dedicated to genome processing. De novo pathways for lipids, cofactors, amino acids and nucleotides are absent from the genome. Nutrient transfer from host to $N$. equitans likely occurs through direct membrane to membrane contact that has been observed by electron microscopy (Huber et al. 2002; Jahn et al. 2008).

Two nanoarchaeotal genomes have recently been sequenced from Yellowstone National Park (YNP). Nanopusillus acidilobi genome has been fully sequenced (Wurch et al. 2016) and Nst1 consists of 7 contigs (Podar et al. 2013). N. acidilobi and Nst1 share extensive protein synteny and a SSU rRNA gene identity of 97-98\%. (Wurch et al. 2016). The YNP nanoarchaeotes also have highly reduced genomes and share many split genes with $N$. equitans. Differing from the marine lineage, the terrestrial genomes contain archaellum genes and a near complete gluconeogenesis pathway, constituting about a $23 \%$ increase in genome size relative to $N$. equitans. 
Unlike the Nanoarchaeota, no korarchaeote has been successfully cultured, but a complete genome was assembled from a stable complex enrichment from Obsidian Pool, YNP (Elkins et al. 2008). Candidatus Korarchaeum cryptofilum contains many incomplete pathways which may be part of the reason that it has eluded isolation (Elkins et al. 2008). Pathways for purines and several cofactors are incomplete. For carbon and energy, $\mathrm{Ca}$. K. cryptofilum contains an abundance of peptide transporters and amino acid fermentation pathways.

A korarchaeotal draft genome from a terrestrial hot spring in Little Hot Creek (LHC), California was recently sequenced and used for comparisons in this study (Saw et al. 2015). Alignment of the LHC korarchaeotal SSU rRNA gene to Candidatus Korarchaeum cryptofilum revealed a 97\% similarity in genomic sequence. Saw et al. (2015) reported a genome of similar size and GC content, but did not report any genomic insights from their assembled metagenome.

In order to expand the genomic diversity of Nanoarchaeota and Korarchaeota, I characterized a nanoarchaeotal and a korarchaeotal genome from a New Zealand hot spring, by combining culture dependent and independent techniques.

\section{Methods}

\section{Samples and Sequencing}

A thermal spring sediment sample was collected from Tikitere (NZ13:

$38^{\circ} 03^{\prime} 47^{\prime} \mathrm{S}, 176^{\circ} 21^{\prime} 39^{\prime} \mathrm{E}$, pH 6.0 and $74^{\circ} \mathrm{C}$ ) in Rotorua, New Zealand. Enrichments were obtained in a modified DSMZ 88 media (per liter): $1.30 \mathrm{~g}\left(\mathrm{NH}_{4}\right)_{2} \mathrm{SO}_{4} ; 0.28 \mathrm{~g} \mathrm{KH}_{2} \mathrm{PO}_{4}$; $0.12 \mathrm{~g} \mathrm{MgSO}_{4} ; 0.07 \mathrm{~g} \mathrm{CaCl}_{2} \cdot 6 \mathrm{H}_{2} 0 ; 4 \mathrm{mg} \mathrm{FeCl}{ }_{3} \cdot 6 \mathrm{H}_{2} 0 ; 4.5 \mathrm{mg} \mathrm{Na} 2 \mathrm{~B}_{4} \mathrm{O}_{7} \cdot 10 \mathrm{H}_{2} 0 ; 2.00 \mathrm{~g}$ 
$\mathrm{H}_{2} \mathrm{~S}_{2} \mathrm{O}_{3}$, supplemented with vitamins (Ferguson et al. 1983), $0.5 \mathrm{~g}$ yeast extract and $0.5 \mathrm{~g}$ trypticase. The $\mathrm{pH}$ was adjusted to 6.0 and incubated at $80^{\circ} \mathrm{C}$ under an atmosphere of $\mathrm{N}_{2}: \mathrm{CO}_{2}(80 \%: 20 \%)$. Genomic DNA was extracted from the environmental sample using the Ultraclean Soil DNA isolation kit (MoBio Laboratories) and enrichment cultures using the DNA Tissue Kit (Qiagen) (K. Brileya, personal communication). Purified DNAs were sent to Oak Ridge National Laboratory for library preparation and metagenomic sequencing. DNA was sequenced on an Illumina MiSeq platform and generated 35 million paired-end reads (2x250bp) (Table 1). Using Trimmomatic, pairedend reads were trimmed for adapter sequences and quality (Bolger et al. 2014). The quality thresholds set discarded bases whose 4-base average fell below a Phred score of 20 and removed reads shorter than $50 \mathrm{bp}$.

\section{Community Composition}

The microbial compositions of the environmental and enrichment samples were compared by extracting the SSU rRNA reads. SortMeRNA v2.0 identified candidate SSU rRNA reads by comparing the reads against aligned SSU reference databases with an evalue threshold of $10^{-5}$ (Kopylova et al. 2012). The reads were then classified using SINA v1.2.11 and a minimal 70\% identity threshold against the small subunit Ref NR 99 128 database (Pruesse et al. 2012).

\section{Assembly and Binning}

Reads from the three NZ13 datasets were concatenated, interleaved and coassembled (Albertsen et al. 2013) de novo using IDBA v1.1.0 (Peng et al. 2012), which resulted in one set of 57,040 contigs. The three sets of reads were mapped back to the 
contigs individually using Bowtie2 v2.2.5 and Samtools v1.2 (Li et al. 2009; Langmead et al. 2012). The assembly was binned using GroopM v0.3.3 differential binning program, which incorporated the assembly and read mapping data (Figure 14) (Imelfort et al. 2014). The contigs were binned using two different thresholds. The nanoarchaeotal contigs were binned using a 1,000 bp cutoff, while the korarchaeotal contigs were binned using a 1500 bp cutoff.

\section{Refinement of nanoarchaeotal and korarchaeotal bins}

Some contigs did not bin during the GroopM process despite using GroopM's unbinned contig recruitment tool. Unbinned contigs were classified using BLASTp v2.2.28 (Camacho et al. 2009). Unbinned contigs with nanoarchaeotal or korarchaeotal BLASTp results were added to their respective bins. Reads were again mapped back to each bin individually and re-assembled using IDBA (Langmead et al. 2012; Peng et al. 2012). The nanoarchaeotal and korarchaeotal assemblies were then individually binned, this time using MaxBin v1.4.5 (Wu et al. 2014). Bins were manually curated using BLASTp, Geneious and coverage data (Kearse et al. 2012). CheckM was used to assess bins for contamination and completion (Parks et al. 2015).

\section{Genome annotation}

The two draft genomic bins (Nanoarchaeota and Korarchaeota) were annotated using Prodigal, MALT, MEGAN5, KEGG, Infernal and HMMR3 software. The protein files, generated by Prodigal v2.6.2, were ran through MALT using default parameters, limiting search results to bit-scores greater than 50 (Hyatt et al. 2010; Huson et al. 2016). The BLASTp output from MALT was imported into MEGAN5 utilizing the KEGG 
mapping software (Aoki-Kinoshita et al. 2007). Protein domains were searched using HMMR3 against the Pfam and TIGRfam databases using trusted cutoffs (Eddy 1998; Haft et al. 2003; Punta et al. 2012). Additionally, some proteins were manually uploaded to InterproScan 5 for further assessment (Jones et al. 2014). To search for RNA motifs, the DNA sequences were processed using Infernal against the Rfam database, once again using trusted cutoffs (Griffiths-Jones et al. 2003). For arCOG analysis, BLASTp was utilized to compare the protein sequences against the curated arCOG database using an evalue of $10^{-5}$ and keeping the top result for each protein sequence (Makarova et al. 2015b). Genome plots and genome similarity plots were generated using ClicO FS and finalized through the Circos software (Cheong et al. 2015; Krzywinski et al. 2009). Genome comparisons

Available nanoarchaeotal and korarchaeotal genomes were used for comparisons against the New Zealand draft genomes. Two complete nanoarchaeotal genomes were available: N. equitans and N. acidilobi (Waters et al. 2003; Wurch et al. 2016). A third draft nanoarchaeotal genome, Nst1, is a close relative to $N$. acidilobi based on the SSU rRNA alignment (Podar et al. 2013). Ca. K. cryptofilum is the only complete korarchaeotal genome (Elkins et al. 2008). However, metagenomic sequence data was provided to our lab from the Hedlund lab (University of Nevada, Las Vegas) which contained korarchaeotal reads from a hot spring sediment sample in Little Hot Creek, California (Saw et al. 2015). The reads were processed in a similar manner as the New Zealand nanoarchaeotal and korarchaeotal genomes. All genomes were analyzed using the same processing analysis pipeline as the New Zealand genomes. 


\section{Results}

\section{Community Analysis}

From the three metagenomes, a total of 46,000 SSU rRNA reads were classified (Figure 1). The enrichment cultures were enriched in crenarchaeotes. Nanoarchaeotal and korarchaeotal sequences were detected in all three samples.

\section{Nanoarchaeota}

The draft nanoarchaeotal genome (NCBI Biosample ID: SAMN05172240) is 532,337 bp and consists of 52 contigs with an average GC content of $32.6 \%$ (Table 2). Completeness, based on lineage specific marker genes, is estimated at 99\% (Table 2). The draft genome encodes for 35 tRNAs throughout 18 contigs. Transfer RNAs (tRNA) aspartic acid, cysteine and glutamine were not detected. The large and small subunit rRNAs are on the same contig (Nnz13_1) separated by six protein coding genes and the 5S rRNA is on contig Nnz13_39 (Figure 2).

The nanoarchaeotal SSU rRNA gene was assembled individually, using only nanoarchaeotal SSU rRNA reads from NZ13en-R and NZ13en-P. The de novo SSU rRNA gene was aligned with a clone library sequence and they are $99.9 \%$ similar (Figure $15)$.

Genomic comparisons among the nanoarchaeotal genomes reveal a closer relationship between the YNP nanoarchaeotal genomes than to N. equitans (Figure 3; Table 9). The NZ13 nanoarchaeote and the YNP nanoarchaeotes SSU rRNA genes share an $88 \%$ identity, likely forming a distinct family within the terrestrial Nanoarchaeota (Table 7). N. equitans and the NZ13 nanoarchaeote share an 84\% SSU rRNA identity. Synteny plots show a higher percentage of protein homology between N. acidilobi-NZ13 
than between N. equitans-NZ13 (Figure 4).

The NZ13 nanoarchaeotal genome shares several genomic features with other nanoarchaeotes, such as a reduced genome, cis-spliced tRNAs and split genes (Table 3). However, there are differences among the common core of split genes. Glu-tRNA ${ }^{\text {Gln }}$ amidotransferase has a conserved split site within $N$. equitans and $N$. acidilobi (Waters et al. 2003; Podar et al. 2013; Wurch et al. 2016) while the gene is not split in the NZ13 nanoarchaeote (Figure 16). Though not all split genes are shared, glucosamine-fructose6-phosphate aminotransferase (GFAT) is unique to the NZ13 nanoarchaeote (Table 3). If functional, GFAT can catalyze the reaction of fructose-6-phosphate and glutamine to produce glutamate.

Only two other genes, $\operatorname{trk} A$ and $\operatorname{trkH}$, are unique to the NZ13 nanoarchaeotal genome. They are a low affinity potassium uptake transport system common in Archaea (Figure 5) (Kraegeloh et al. 2005). TrkH is a transmembrane protein responsible for potassium translocation and $\operatorname{trk} A$ is the cytoplasmic membrane receptor.

The NZ13 nanoarchaeotal genome share many metabolic features with the terrestrial nanoarchaeotal genomes, which are absent in N. equitans (Figure 3). The NZ13 nanoarchaeotal genome encodes for the gluconeogenesis and glycolysis pathways (Figure 5). Two gluconeogenic genes, glyceraldehyde-3-phosphate dehydrogenase and phosphoglycerate kinase were not present in the draft genome. All other gluconeogenic genes are present, including those for the complete synthesis and breakdown of glycogen. In the glycolytic pathway 6-phosphofructokinase, $\mathrm{NADP}^{+}$dependent nonphosphorylating glyceraldehyde-3-phosphate dehydrogenase (GAPN) and pyruvate kinase were not 
identified.

Another shared physiology between the NZ13 nanoarchaeote and the terrestrial nanoarchaeotes are the presence of archaellum genes. All essential subunits for archaellum assembly and function have been identified in the genome (Figure 6). Additionally, the genes are not contained in an operon, but are fragmented throughout the genome.

A partial CRISPR-cas system was identified in the NZ13 nanoarchaeotal genome (Figure 5; Table 9). The NZ13 nanoarchaeotal genome encodes casl, cas4 and cas6.

\section{Korarchaeota}

The draft genome of the NZ13 korarchaeote (NCBI Biosample ID:

SAMN05172504) is $1.375 \mathrm{Mbp}$ and consists 148 contigs with an average GC content of $56.7 \%$ (Table 4). The genome is estimated to be $91 \%$ complete (Table 4). Only a partial 23S rRNA and complete 5S rRNA gene are located on the draft genome, while there are a full complement of tRNA genes (Figure 7).

A de novo assembly of the NZ13 korarchaeotal SSU rRNA gene is presented in Figure 17. A large portion of the SSU rRNA gene was assembled from the environmental SSU rRNA reads (NZ13). The assembled SSU rRNA sequence is $99.39 \%$ similar to a clone library sequence obtained previously (R. Davis, unpublished results) (Figure 18). The NZ13 korarchaeote SSU rRNA sequence shares a $91 \%$ nucleotide similarity to $\mathrm{Ca}$. K. cryptofilum (Table 10). A synteny plot also shows a high level of protein homology between the NZ13 korarchaeotal and $C a$. K. cryptofilum genomes (Figure 8).

The NZ13 korarchaeotal genome encodes over 100 transport genes (Table 11). 
There are transporters for many cations, anions and ionic compounds. Two transport genes, the sodium/hydrogen antiporters, are not present in the other korarchaeotal genomes (Figure 9). However, most transporters are classified as $\mathrm{ABC}$ transporters for amino acids or peptides, with only one gene being classified as a sugar transporter.

The NZ13 korarchaeotal genome encodes an extensive set of amino acid pathways. Amino acid degradation pathways proceed through pyruvate or the partial TCA cycle to enter the central metabolism (Figure 9). Citrate synthase, aconitase and isocitrate dehydrogenase were not identified in the genome. Gluconeogenesis and glycolysis pathways proceed through the archaeal Embden-Meyerhof-Parnas pathway, however, a hexokinase was not identified in the genome. Furthermore, the pentose pathway proceeds through the ribulose monophosphate pathway for pyrimidine synthesis. Purines cannot be synthesized de novo, but purine salvage and interconversion pathways are present.

The NZ13 korarchaeotal genome contains an ATP synthase to utilize a proton motive force for generating ATP (Figure 9). Proton translocation can occur through NADH dehydrogenase. The electrons may then be transferred to a menaquinone and terminal electrons can be shuttled to a NiFe hydrogenase. Electrons could also flow through formate dehydrogenase to heterodisulfide reductase (Costa et al. 2010). The subunits of $\mathrm{F}_{420}$ reducing hydrogenase are also present, but the genes for $\mathrm{F}_{420}$ synthesis were not detected. Lastly, two genes are present for cytochrome $b d$ oxidase subunit I.

Several coenzyme biosynthesis pathways are incomplete. For instance, the coenzyme pathway gene, phopantothenoylcysteine synthetase/decarboxylase, was not 
identified. Menaquinone biosynthesis probably proceeds through the archaeal alternate mevalonate pathway but phosphomevalonate decarboxylase was not detected. While the pathway for riboflavin was not detected, there is a riboflavin transport gene. Other incomplete coenzyme pathways include those for pterin, lipoate and porphyrin.

\section{Discussion}

Pairing environmental and enrichment samples with differential coverage binning of metagenomic data allows for the recovery of low abundance, high quality microbial genomes (Albertsen et al. 2013). These methods bypass the arduous task of isolating an organism for sequencing, producing the addition of the nearly complete nanoarchaeotal and korarchaeotal genomes. The genomes demonstrate the conserved physiologies among terrestrial members of two basal archaeal lineages.

Aside from the genomes, the enrichment cultures in this study provide insights into another nanoarchaeotal-host system. In line with other known nanoarchaeotal hosts (Waters et al. 2003; Podar et al. 2013; Wurch et al. 2016), the NZ13 cultures were highly enriched for crenarchaeotal sequences. However, N. equitans, N. acidilobi and Nst1 utilize three host from different crenarchaeotal orders, therefore only a coculture or single cell sequencing could resolve the potential NZ13 crenarchaeotal host.

Previous studies have shown the archaellum genes are expressed in the YNP nanoarchaeotes, but their purpose remains uncertain (Podar et al. 2013; Wurch et al. 2016). The archaellum genes are typically located in a single operon (Jarrell et al. 2012), but they are fragmented in the nanoarchaeotal genomes thus far studied. Their suggested function would allow the nanoarchaeotes to move between hosts or have involvement in 
the host attachment process. However, archaella are predicted to function using ATP (Streif et al. 2008), which the nanoarchaeotes must obtain from their host. Therefore, the use of archaella during detachment from their host would drain ATP reserves, effectively making the nanoarchaeotes non-motile. If the archaella are functional while attached to their host, they could potentially help the nanoarchaeotes and the host to move to a more nutrient rich location. Evidence for this symbiosis comes from the physiologies of the hosts. Archaella have been observed on the host of N. equitans, Ignicoccus hospitalis (Paper et al. 2007; Podar et al. 2008), so the loss of archaellum genes in N. equitans would be expected. Conversely, the terrestrial nanoarchaeotes have retained their archaellum genes while their host do not have archaella. For example, the host of Nst1 is predicted to have lost their archaellum genes (Podar et al. 2013), while the host of $N$. acidilobi is from a non-motile genus (Prokofeva et al. 2000; Mardanov et al. 2010; Wurch et al. 2016). The different coevolutionary paths, possibly dependent on host motility, may explain the terrestrial retention of their archaellum genes.

Another shared nanoarchaeotal terrestrial trait is carbohydrate metabolism. The NZ13 nanoarchaeotal genome does not encode for two glycolytic enzymes that are present in N. acidilobi and Nst1 (Podar et al. 2013; Wurch et al. 2016). These genes could be encoded in the completed gene, but phosphofructokinase is absent from all genomes (complete and draft), leaving the glycolytic pathway unable to proceed past fructose-6-phosphate (F6P). In the NZ13 nanoarchaeote, the breakdown of glycogen could proceed through F6P utilizing the split gene, GFAT, to produce glutamate. Glutamate is five times more prevalent than glutamine in the predicted NZ13 
nanoarchaeotal proteome, a common occurrence in Archaea (Table 8) (Schmidt et al. 2012).

A common core of split genes is found within all nanoarchaeotal genomes (Table 3). The similarities of split genes suggest that the genome reduction process began before the terrestrial and marine lineages diverged. As there are split genes unique to each genome, it is likely that genome streamlining continued to occur after each lineage diverged. (Waters et al. 2003; Podar et al. 2013; Wurch et al. 2016).

One shared suite of genes between N. equitans and the NZ13 nanoarchaeotal genome are the CRISPRs. The three NZ13 nanoarchaeotal cas genes are associated with CRISPR type I-B, the same proteins found in N. equitans (Waters et al. 2003; Randau 2012; Makarova et al. 2015a). Although Munson-McGee (2015) postulated that $N$. equitans likely gained their CRISPR genes through horizontal transfer, rather than Nst1 (or $N$. acidilobi) losing their CRISPR system through genome reduction. The presence of a similar CRISPR system found in the NZ13 nanoarchaeote suggests the YNP nanoarchaeotes have likely lost their viral defense system.

Like the Nanoarchaeota, the terrestrial korarchaeotes have similar metabolisms (Figure 10). Only two transport genes differentiates the NZ13 korarchaeotal genome from $\mathrm{Ca}$. K. cryptofilum or the LHC korarchaeote. In all three genomes, the abundance of peptide transporters and amino acid degradation pathways demonstrates the korarchaeotes are amino acid fermenters. Additionally, the only amino acids the korarchaeotal genomes cannot synthesize de novo are histidine and tryptophan.

Other incomplete pathways in the korarchaeotal genomes involve coenzymes and 
a menaquinone. The $C a$. K. cryptofilum coenzyme A synthesis pathway is missing one gene, phopantothenoylcysteine synthetase/decarboxylase and was not identified in the NZ13 korarchaeote (Elkins et al. 2008). With a large portion of the genomes categorized as having an unknown function, there is the possibility of an undiscovered enzyme that can complete the pathway or even an undiscovered pathway unique to Archaea.

Similarly, a carboxy-lyase gene may complete the menaquinone biosynthesis pathway. If not, the abundance of transport genes within the korarchaeotal genomes may allow for efficient scavenging of nutrients.

Energy metabolism within the korarchaeotal genomes is also an area of uncertainty. Methanogen-like genes, such as the heterodisulfide reductase and $\mathrm{F}_{420}$ hydrogenase, have been found in non-methanogens, but their functional role has not been determined (Lee et al. 2017). Typical methanogen genes are not found in the korarchaeotal genomes, such as mcrA or pathways for coenzyme M, B and $\mathrm{F}_{420}$ synthesis. Moreover the presence of the cytochrome $b d$ oxidases are not unusual in anaerobes (Giuffrè et al. 2014), but the korarchaeotal genomes only contain two copies of subunit I. If functional they could provide the korarchaeotes with oxidative stress protection. Biochemical studies of korarchaeotes or other non-methanogens are needed to fully understand early archaeal energy metabolism.

Both the NZ13 nanoarchaeote and NZ13 korarchaeote contain transport genes related to osmotic regulation. The NZ13 nanoarchaeote contains the $\operatorname{trk} A H$ genes that import potassium. The NZ13 korarchaeote contains a $\mathrm{Na}^{+} / \mathrm{H}^{+}$antiporter, which studies have shown can also uptake potassium (Verkhovskaya et al. 1995; Southworth et al. 
2001). In halotolerant organisms, internal potassium levels are maintained in high concentrations relative to external sodium levels (Martin et al. 1999). These potassium transport genes may provide salt tolerance to the NZ13 nanoarchaeotes and NZ13 korarchaeotes.

Recently metagenomic sequencing has revealed extensive microbial diversity, including many deep-branching archaeal lineages (Rinke et al. 2013; Spang et al. 2015; Castelle et al. 2015). Some of these lineages have relatively small genomes and lack core biosynthetic pathways, similar to the Nanoarchaeota and Korarchaeota (Baker et al. 2010; Castelle et al. 2015). As a result they may form symbiotic relationships or be efficient scavengers. With this in mind, isolation of symbiotic and uncultivated archaeal lineages, such as the Nanoarchaeota and Korarchaeota, are needed to better understand archaeal symbiosis, metabolism and evolution. 
Table 1. Summary of raw data from the three metagenomes and co-assembly. NZ13 is the environmental sample. NZ13en-R and NZ13en-P are enrichment samples.

\begin{tabular}{|c|c|c|c|}
\hline Sequence Data & NZ13 & NZ13en-R & NZ13en-P \\
\hline Number of Paired-end Reads & $12,517,520$ & $10,038,732$ & $12,316,813$ \\
\hline Total Base Pairs (Mbp) & 5,158 & 4,496 & 5,570 \\
\hline Average Read Length (bp) & 206 & 224 & 226 \\
\hline \multicolumn{4}{|l|}{ SSU rRNA Breakdown } \\
\hline SSU rRNA Read Counts & 19,088 & 11,878 & 15,034 \\
\hline Archaea SSU rRNA & $69.2 \%$ & $66.5 \%$ & $99.7 \%$ \\
\hline Bacteria SSU rRNA & $29.6 \%$ & $33.4 \%$ & $0.2 \%$ \\
\hline Eukarya SSU rRNA & $1.2 \%$ & $0.1 \%$ & $0.0 \%$ \\
\hline Assembly Data & & Co-assembly & \\
\hline Total Co-assembly Size (bp)* & & $137,989,742$ & \\
\hline Total Number of Contigs* & & 57,040 & \\
\hline $\mathrm{N} 50(\mathrm{bp})^{*}$ & & 2,716 & \\
\hline Longest Contig (bp) & & 107,757 & \\
\hline
\end{tabular}




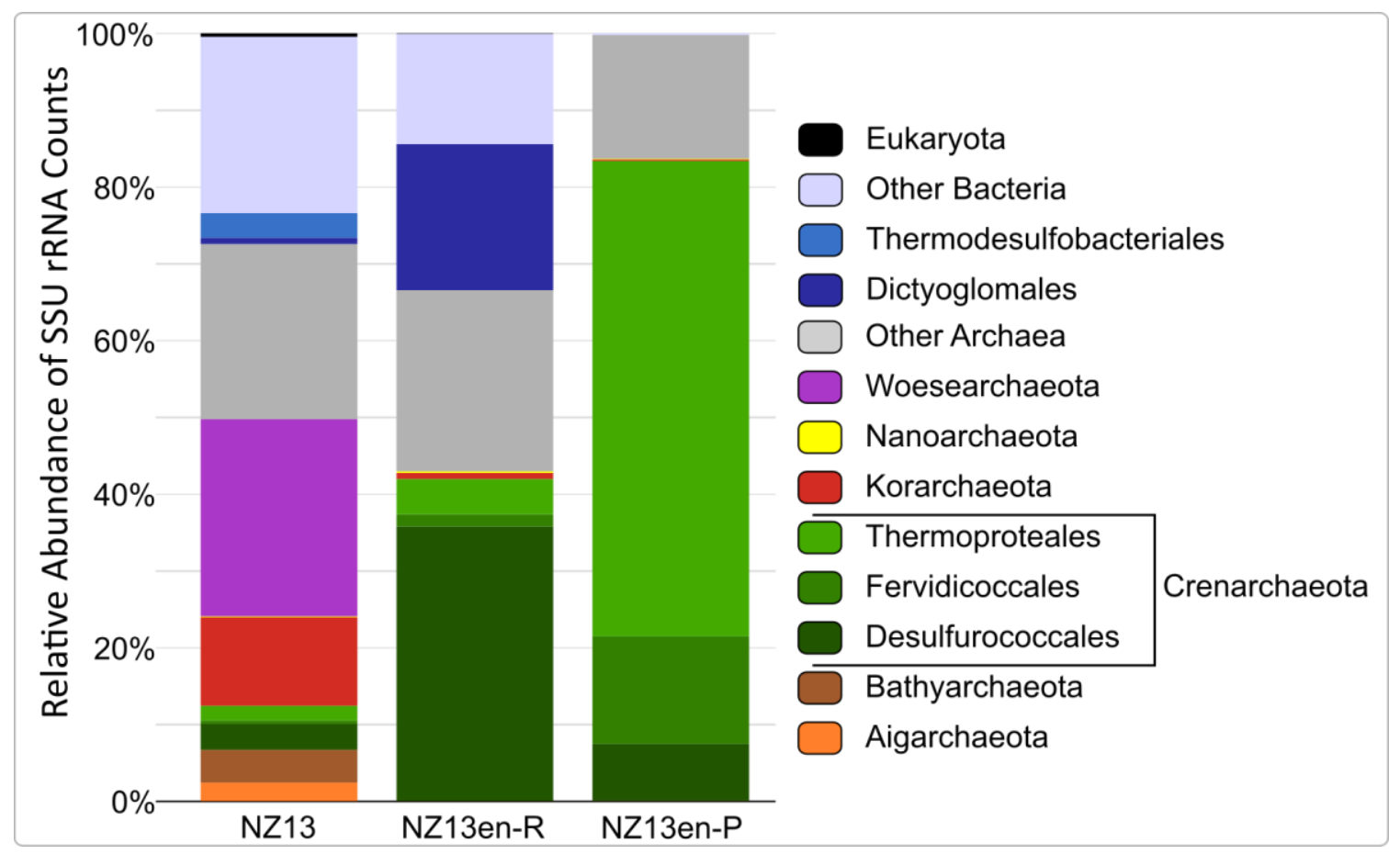

Figure 1. Relative abundance of SSU rRNA trimmed read counts from NZ13, NZ13en-R and NZ13en-P. Only shown are Family level classification with higher than $1 \%$ relative abundance, all other reads are grouped into their respective domain. Reads were classified against the SILVA SSU Ref NR128 database. 
Table 2. Comparison of general features of nanoarchaeotal genomes. Predicted proteins were generated using Prodigal (Hyatt et al. 2010). Completeness and contamination were assessed using CheckM (Parks et al. 2015).

\begin{tabular}{lcccc}
\hline Features & NZ13 Nanoarchaeota & Nanopusillus acidilobi & Nst1 & Nanoarchaeum equitans \\
\hline Genome Length (bp) & 532,337 & 605,887 & 593,789 & 490,885 \\
No. of Contigs & 52 & - & 7 & - \\
Longest Contig (bp) & 88,440 & - & 192,298 & - \\
G+C\% & $33 \%$ & $24 \%$ & $24 \%$ & $32 \%$ \\
Predicted Proteins & 647 & 665 & 654 & 583 \\
rRNA & 3 & 3 & 3 & 3 \\
tRNA & 35 & 43 & 41 & 43 \\
Completeness & $99 \%$ & $100 \%$ & $98 \%$ & $100 \%$ \\
Contamination & 0 & 0 & 0.93 & 0 \\
\hline
\end{tabular}



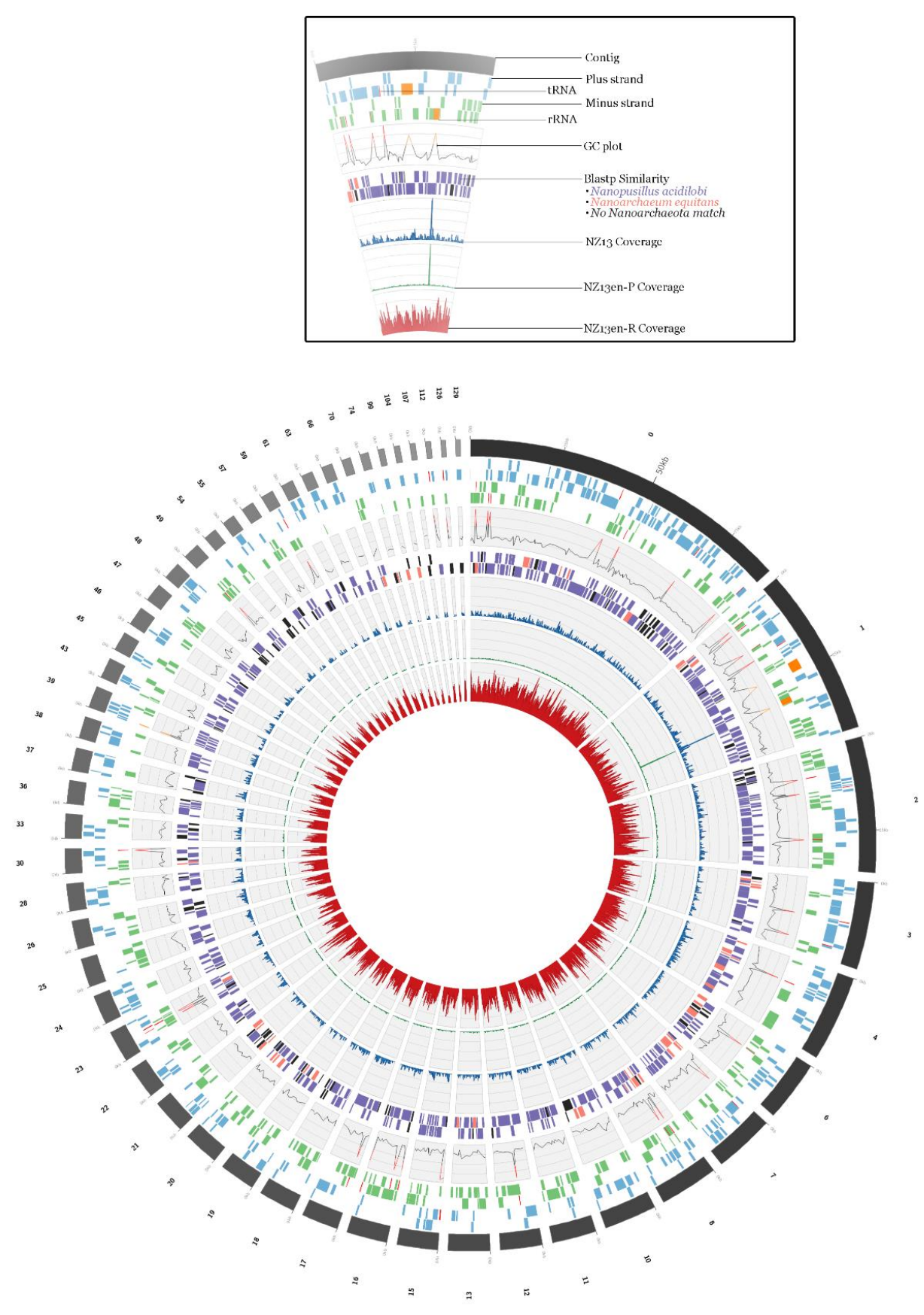

Figure 2. NZ13 nanoarchaeotal genome plot. ORFs, GC\% and RNAs were predicted by Prodigal and Rfam. BLASTp was used to generate similarity tiles. Bowtie2 and Samtools were used to assess coverage. 


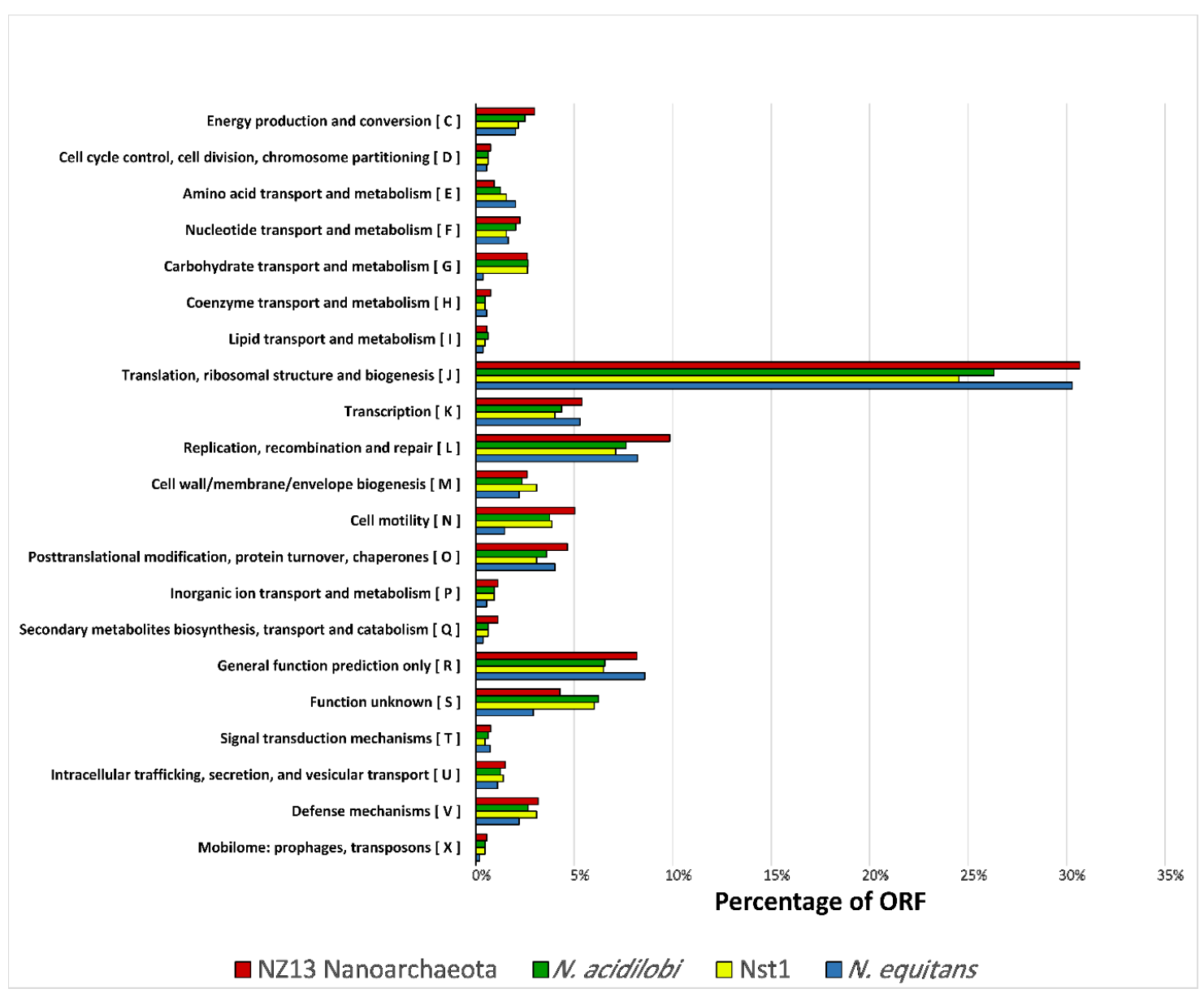

Figure 3. Relative abundance of arCOG categories from ORFs in nanoarchaeotal genomes. The arCOG categories A, B, W, Y and Z did not have any matches. Not shown are proteins that were labeled unclassified. Relative abundances of unclassified proteins are expressed as a percentage of ORFs: NZ13 Nanoarchaeota (10.41\%), $N$. acidilobi (22.59\%), Nst1 (25.46\%) and N equitans (24.45\%). 

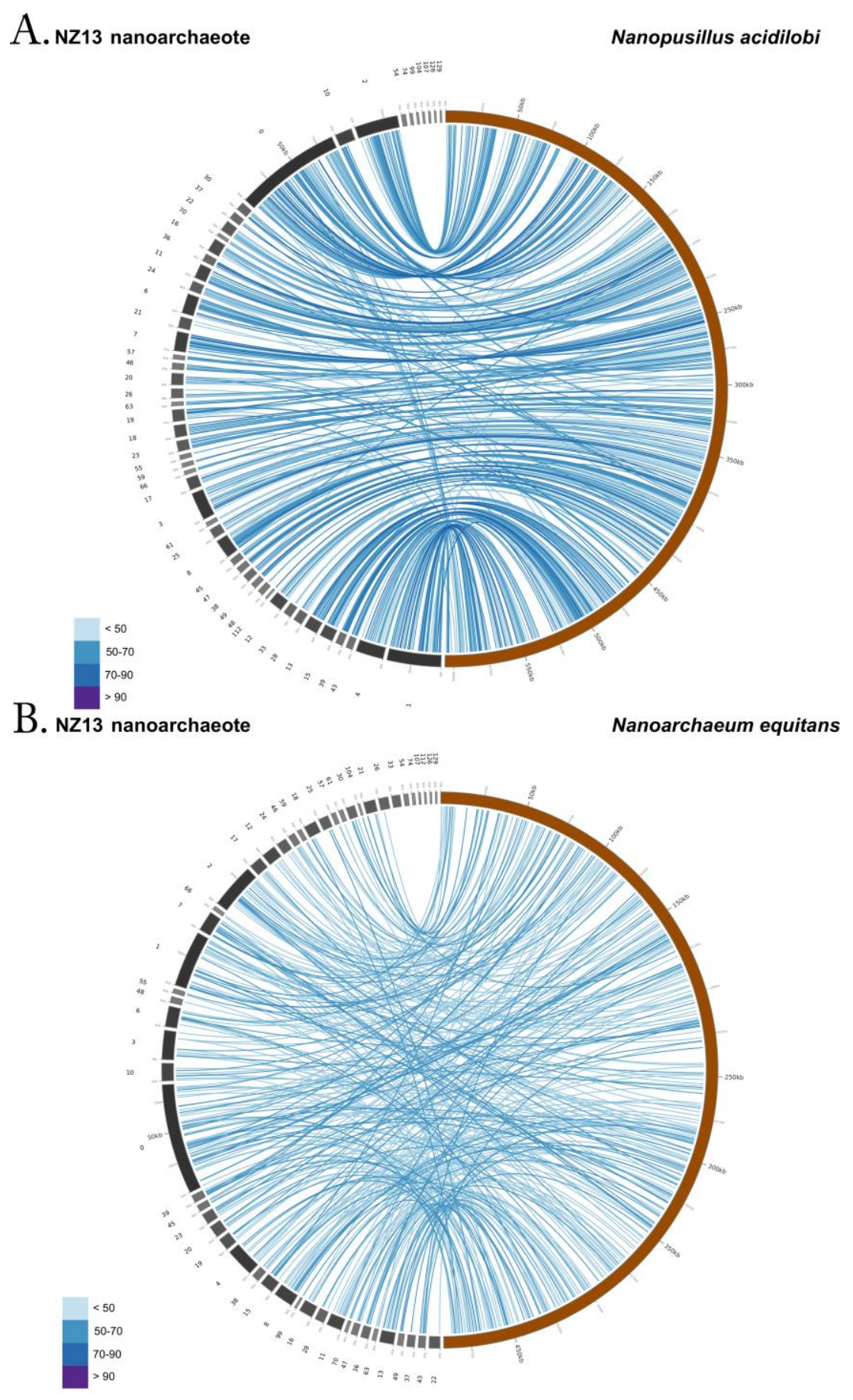

Figure 4. NZ13 nanoarchaeotal BLASTp synteny plots. A. NZ13 nanoarchaeotal protein to protein comparison against Nanopusillus acidilobi. B. NZ13 nanoarchaeotal protein to protein comparison against Nanoarchaeum equitans. Connecting lines represent shared proteins between the genomes, the color represents the percent of amino acid identity. 
Table 3. Overview of nanoarchaeotal similarities and differences: shared split proteins, trans and cis-spliced tRNA and genomic features.

\begin{tabular}{|c|c|c|c|c|}
\hline Split proteins & $\begin{array}{c}\text { NZ13 } \\
\text { Nanoarchaeota }\end{array}$ & $\begin{array}{l}\text { Nanopusillus } \\
\text { acidilobi }^{*}\end{array}$ & Nst1 ${ }^{*}$ & $\begin{array}{l}\text { Nanoarchaeum } \\
\text { equitans }^{*}\end{array}$ \\
\hline Reverse gyrase & Nnz13 13_3-3_10 & Nps 2030-2705 & Nst1 337-402 & NEQ 318-434 \\
\hline Glu-tRNA ${ }^{\text {Gln }}$ amidotransferase & Nnz13 0_76 & Nps 2280-3415 & Nst1 197-449 & NEQ245-396 \\
\hline Predicted RNA-binding protein & Nnz13 2_37-1_56 & Nps 30-3140 & Nst1 176-251 & NEQ 438-506 \\
\hline $\begin{array}{l}\text { Archaeosine tRNA-guanine } \\
\text { transglycosylase }\end{array}$ & Nnz13 11_6-1_37 & Nps $1135-3235$ & Nst1 096-232 & NEQ 124-305 \\
\hline RNA polymerase subunit B & Nnz13 0_36-0_35 & Nps 545-550 & Nst1 632-633 & NEQ 156-173 \\
\hline Large helicase-related protein & Nnz13 0_74-1_43 & Nps 745-3195 & Nst1 172-239 & NEQ 003-409 \\
\hline DNA polymerase I & Nnz13 25_5 & Nps 2105 & Nst1 417 & NEQ 068-528 \\
\hline Topoisomerase I & Nnz13 2_35 & Nps 45 & Nst1 174 & NEQ 045-324 \\
\hline $\begin{array}{l}\text { P-loop ATPase-acetyltransferase } \\
\text { fusion protein }\end{array}$ & Nnz13 3_11 & Nps 2025 & Nst1 401 & NEQ 096-495 \\
\hline Alanyl-tRNA synthetase & Nnz13 10_7 & Nps 290 & Nst1 054 & NEQ 211-547 \\
\hline $\begin{array}{l}\text { Diphthamide synthase sub. } \\
\text { DPH2 }\end{array}$ & Nnz13 1_28-8_15 & Nps 3285-2235 & Nst1 222-440 & NEQ226 \\
\hline $\begin{array}{l}\text { Uncharacterized conserved } \\
\text { protein (arCOG04253) }\end{array}$ & Nnz13 49_7 & Nps 2410 & Nst1 474-480 & - \\
\hline $\begin{array}{l}\text { Glucosamine-fructose-6- } \\
\text { phosphate aminotransferase }\end{array}$ & Nnz13 0_85-0_87 & - & - & - \\
\hline
\end{tabular}

\section{tRNA}

\begin{tabular}{lcccc}
\hline cis-spliced & Ile, Tyr, Met & Ile, Tyr & Ile, Tyr & Ile, Met, Trp, Tyr \\
trans-spliced & - & - & - & iMet, His, Lys, Gln, \\
& & & Glu (2)
\end{tabular}

\section{Genome Features}

Gluconeogenesis-Glycolysis

CRISPR related genes

Polyamine biosynthesis

Archaellum

ATP synthase

Glutamate dehydrogenase

*Annotations from NCBI, Accession numbers: N. acidilobi (CP010514), Nst1 (APJZ00000000), N.

equitans (AE017199)

- absent

+ present

${ }^{\dagger}$ Lacking several subunits 


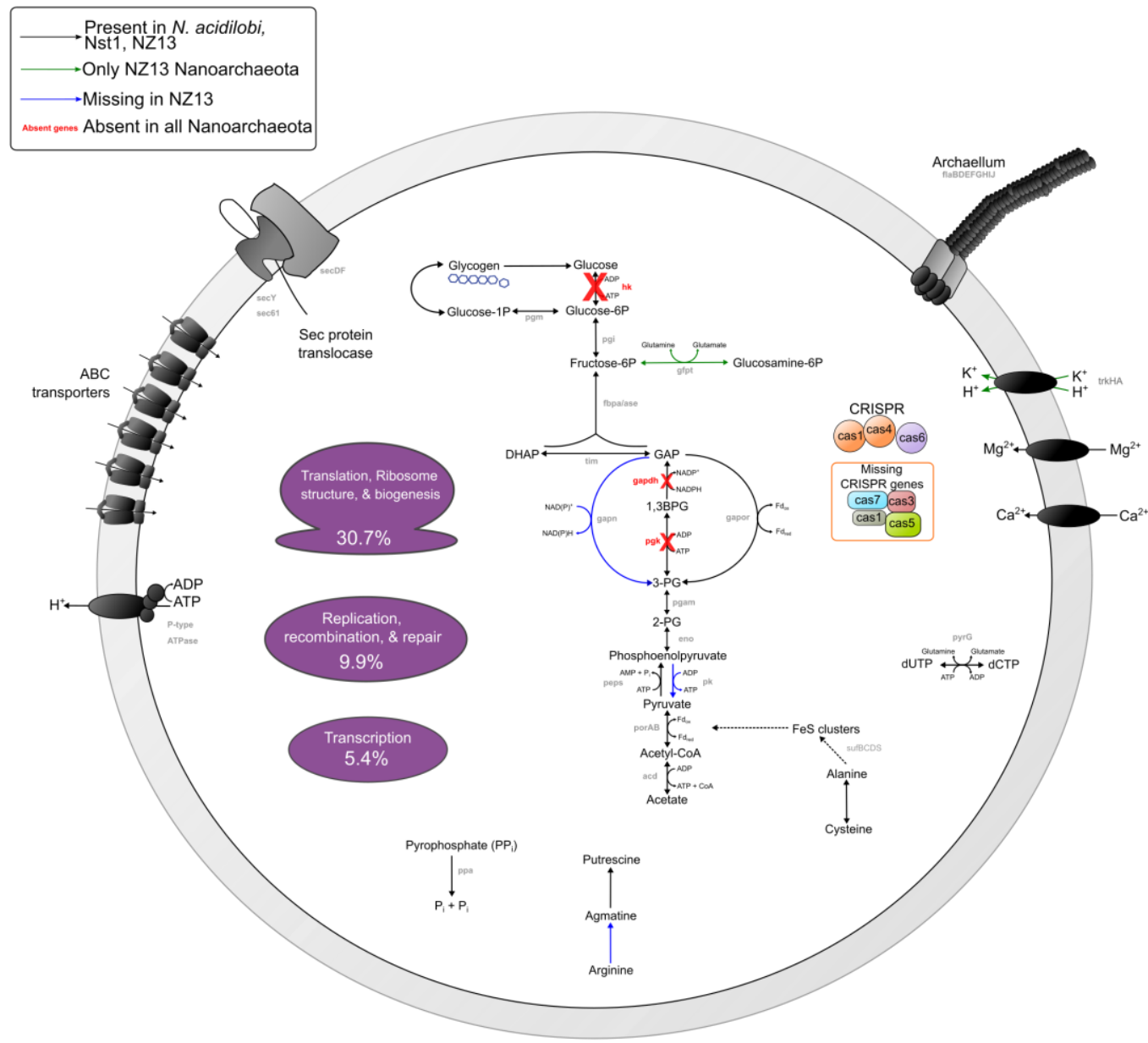

Figure 5. Reconstructed proposed metabolic chart of NZ13 Nanoarchaeota. Percentages for transcription, replication and translation are derived from arCOGs. The CRISPR genes missing here are present in N. equitans. 


\section{NZ13 Nanoarchaeota}

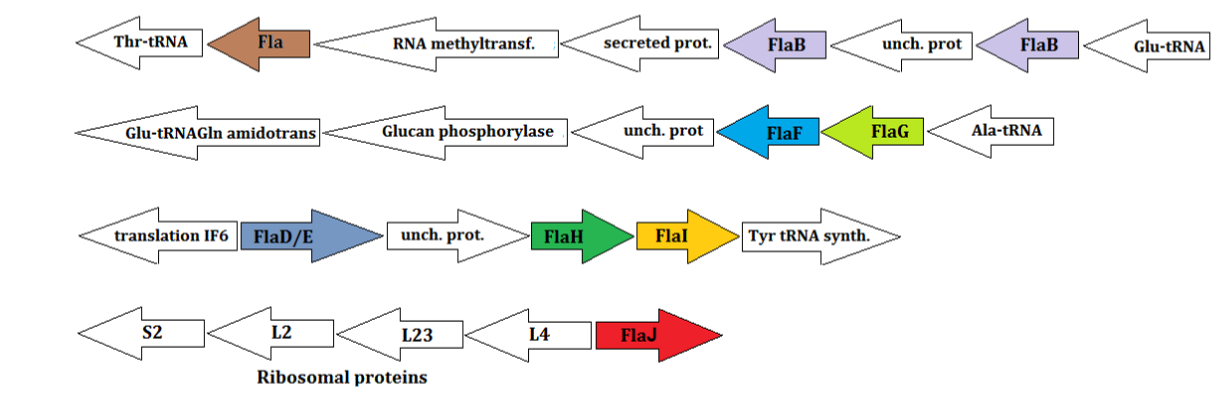

\section{Nanopusillus acidilobi}

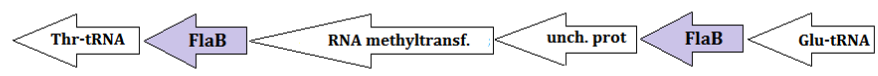

Glu-tRNAGln amidotrans $>$ FlaG $>$ unch. prot $>$ His-tRNA
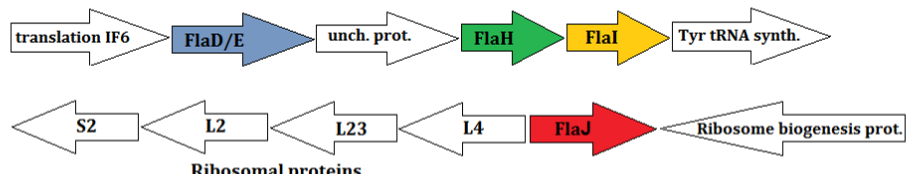

Ribosomal proteins

\section{Nst1}

$<$ Thr-tRNA $<$ FlaB $<$ RNA methyltransf. $<$ unch. prot $<>$ FlaB $<<$

$<$ His-tRNA $<$ unch. prot $<$ FlaF $<$ FlaG $<$ Glu-tRNAGln amidotrans

translation IF6 $>$ FlaD/E

$<S 2<\underset{\text { Ribosomal proteins }}{\mathrm{L} 2}<\underset{\mathrm{L23}}{\mathrm{L} 2}<$

\section{Sulfolobus acidocaldarius DSM 639}

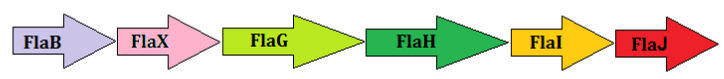

\section{Pyrococcus horikoshi OT3}

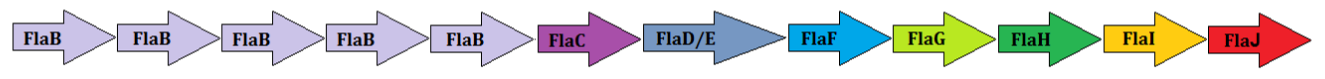

Figure 6. Archaellum gene locations and orientation in the terrestrial nanoarchaeotal genomes: NZ13 Nanoarchaeota, N. acidilobi and Nst1. Sulfolobus and Pyrococcus are shown to illustrate a typical archaellum operon structure. 
Table 4. General features table comparing three korarchaeotal genomes. Predicted proteins were generated using Prodigal (Hyatt et al. 2010). Completeness and contamination were assessed with CheckM (Parks et al. 2015).

\begin{tabular}{lccc}
\hline Features & $\begin{array}{c}\text { NZ13 } \\
\text { Korarchaeota }\end{array}$ & $\begin{array}{c}\text { Candidatus Korarchaeum } \\
\text { cryptofilum }\end{array}$ & $\begin{array}{c}\text { Little Hot Creek } \\
\text { Korarchaeota }\end{array}$ \\
\hline Base Pairs (bp) & $1,374,210$ & $1,590,757$ & $1,358,156$ \\
No. of Contigs & 148 & 1 & 405 \\
Longest Contig (bp) & 107,729 & - & 22,651 \\
G+C\% & $56.7 \%$ & $49.0 \%$ & $47.6 \%$ \\
Predicted Proteins & 1587 & 1683 & 1751 \\
rRNA & $5 \mathrm{~S} ; 23 \mathrm{~S} \dagger$ & $5 \mathrm{~S} ; 16 \mathrm{~S} ; 23 \mathrm{~S}$ & $5 \mathrm{~S}$ \\
tRNA & 41 & 45 & 31 \\
$\quad$ - cis-spliced & Met, Trp & Met, Tyr & Met, Ile \\
Completeness & $90.9 \%$ & $100 \%$ & $88.7 \%$ \\
Contamination & 0.93 & 2.8 & 5.14 \\
\hline
\end{tabular}

$\dagger$ partial 

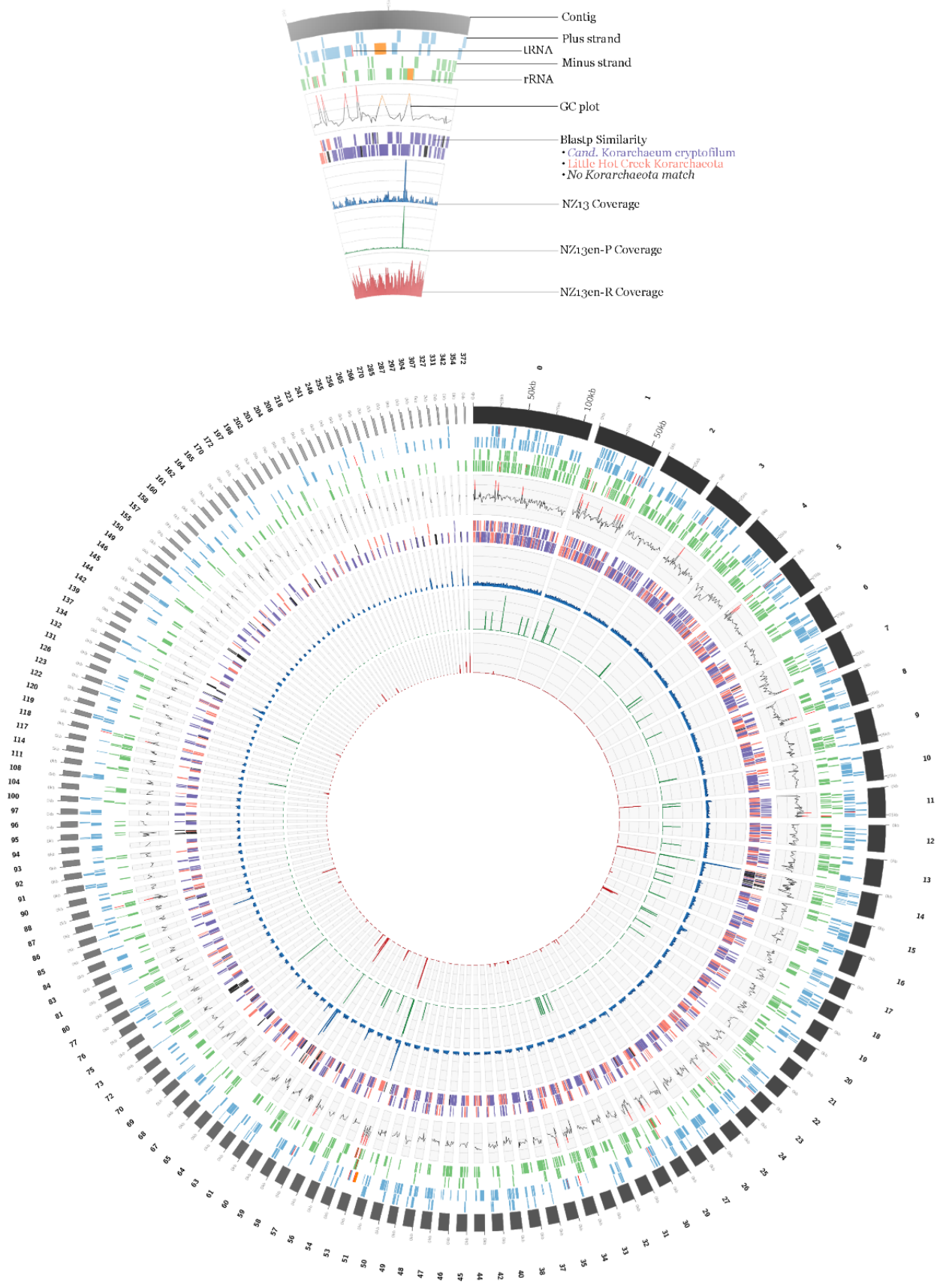

Figure 7. NZ13 korarchaeotal genome map. ORFs, GC\% and RNAs were predicted by Prodigal and Rfam. BLASTp was used to generate similarity tiles. Bowtie 2 and Samtools were used to assess coverage. 


\section{NZ13 korarchaeote}

\section{Candidatus}

Korarchaeum cryptofilum

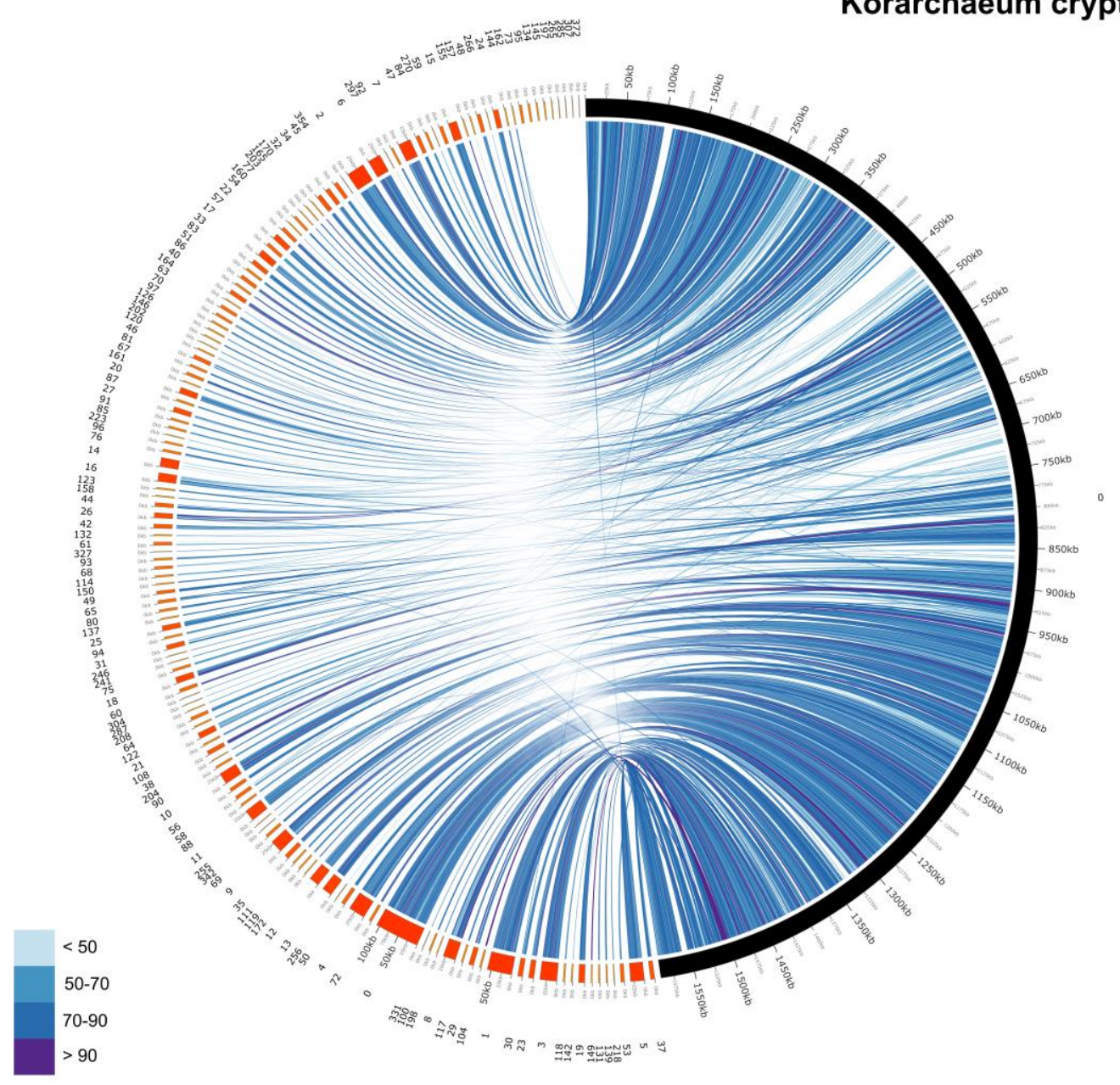

Figure 8. NZ13 korarchaeotal BLASTp synteny plots. NZ13 Korarchaeota protein to protein comparison against Candidatus Korarchaeum cryptofilum. Connecting lines represent shared proteins between the genomes, the color represents the percent of amino acid identity. 


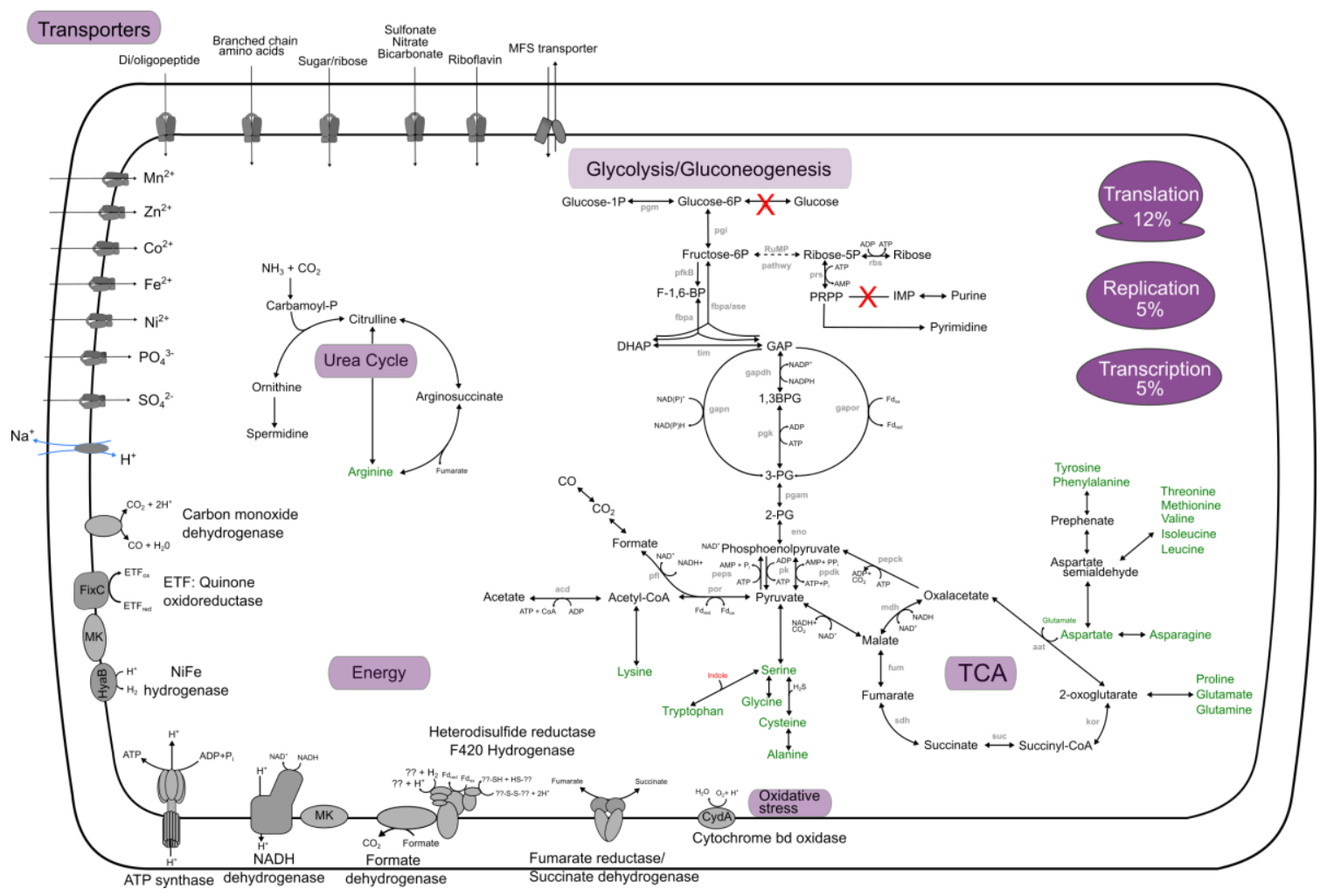

Figure 9. Reconstructed proposed NZ13 korarchaeotal metabolism chart. Amino acids are green, with tryptophan requiring input of indole to be synthesized. A red $\mathrm{X}$ denotes an incomplete pathway. Translation, replication and transcription are percentages from arCOGs. Menaquinone is abbreviated MK. Blue arrows indicate the transport gene found only in the NZ13 korarchaeote. 


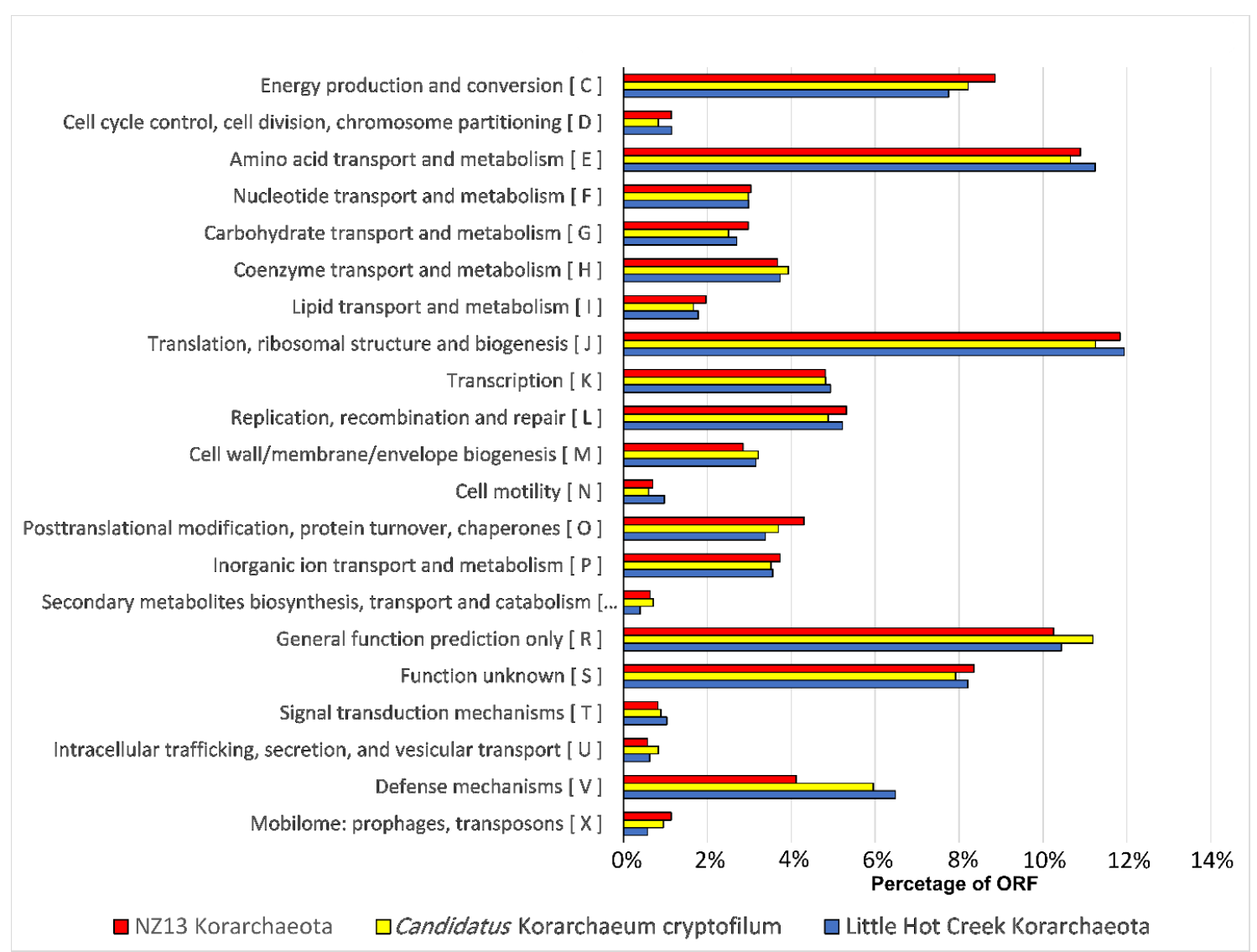

Figure 10. Relative abundance of arCOG categories from ORFs in korarchaeotal genomes. The arCOG categories A, B, W, Y and Z did not have any matches. Not shown are proteins that were labeled unclassified. Relative abundances of unclassified proteins are expressed as a percentage of ORFs: NZ13 Korarchaeota (8.04\%), Ca. K cryptofilum (8.81\%) and Little Hot Creek Korarchaeota (7.75\%). 


\title{
Chapter 3: Microbial Community Composition of Deep-sea Hydrothermal Vents from the Eastern Lau Spreading Center
}

\author{
Abstract \\ Archaea inhabiting hydrothermal vents occupy niches with low $\mathrm{pH}$ and high \\ temperature and pressure. Previous studies have indicated that euryarchaeotal \\ methanogens, Thermococcales and Archaeoglobales were some of the more abundant \\ Archaea from deep-sea hydrothermal vents. In contrast to the above findings, recent data \\ from deep-sea hydrothermal vents along the Eastern Lau Spreading Center (ELSC), a \\ major vent system in the southwestern Pacific, indicates that Crenarchaeota were more \\ abundant and diverse than previously thought. The metagenomes of ten sulfide samples \\ were sequenced and SSU rRNA reads were classified to identify the microbial \\ composition. With the exception of the Vai Lili sample, Epsilonproteobacteria were the \\ most abundant phylum in every sample. Crenarchaeota were the most abundant archaeal \\ phylum in only three of the ten samples, while the Korarchaeota were the most abundant \\ archaeal phylum at Vai Lili. Archaeoglobaceae and Thermococcaceae were the most \\ abundant euryarchaeotal families in several samples.
}

\section{Introduction}

Along the Eastern Lau Spreading Center (ESLC) and Valu Fa Ridge (VFR) the Pacific oceanic plate is subducting under the Indo-Australian plate, generating a spreading center with several hydrothermal vent fields (Martinez et al. 2007). At active

deep-sea hydrothermal vents, Bacteria are the most abundant microbes, with Epsilonproteobacteria often being the dominant lineage (Reysenbach et al. 2000; Corre 2001; Flores et al. 2011; Flores et al. 2012a). While less abundant, the Archaea of deep- 
sea hydrothermal vents represent a rich phylogenetic diversity (Auguet et al. 2010). Until recently, the Euryarchaeota phylum accounted for most of this diversity with members of Archaeoglobales, Thermococcales and DHVE2 (Nercessian et al. 2003; Flores et al. 2011; Takai et al. 2011; Flores et al. 2012a; Flores et al. 2012b). The Crenarchaeota were represented predominately by Desulfurococcales and Thermoproteales, though there are many uncultivated deep-sea hydrothermal vent lineages (Erauso et al. 1993; González et al. 1998; Huber et al. 2000; Takai et al. 2000; Jolivet et al. 2003; Reysenbach et al. 2006). Hydrothermal fluid chemistry has been shown to influence the composition of microbial communities. For example, the presence of methanogens is dependent on high concentrations of $\mathrm{H}_{2}$ (Flores et al. 2011). Flores et al. (2012a) showed that bacterial and archaeal community structure were significantly different when end member hydrothermal fluid was acidic $(\mathrm{pH}<3)$ and had high concentrations of $\mathrm{CO}_{2}$ (Flores et al. 2012). The culture-independent techniques used by Flores et al. $(2011,2012)$ at two separate hydrothermal locations revealed great diversity amongst crenarchaeotal sequences, with many unclassified and uncultured lineages. For example, the acidic hydrothermal fluid hosted a newly detected family for deep-sea vents, Caldisphaeraceae, a thermoacidophilic Crenarchaeota.

The purpose of this study was to determine the microbial community metagenomic composition of ten sulfide samples and to identity the samples that should be further explored for as yet uncultivated bacterial and archaeal genomes. The genomic information and metabolic potentials revealed by this study may help develop media for isolating uncultured microbes, but more importantly, has the potential to show the roles 
these uncultivated microbes may have at deep-sea hydrothermal vents.

\section{Methods}

Sample collection and processing

The spreading center of the Lau back-arc basin is $397 \mathrm{~km}$ and is located between the Lau and Tonga Ridge (Ferrini et al. 2008). A cruise aboard $R / V$ Roger Revelle in April-May 2015 visited the ELSC and VFR. Fifty samples were collected using the ROV Jason II from four vent fields: ABE, Tui Malila, Mariner and Vai Lili. Ten samples were selected for metagenomic analysis (see below) (Figure 11, Figure 19, Table 12). Each rock sample was placed in a biobox until the rock could be brought on deck for processing. On deck, the outer few millimeters of each sample was scraped off and homogenized. DNA was extracted using the Ultra Clean Soil DNA isolation kit (MoBio Laboratories) and stored in multiple cryovials at $-80^{\circ} \mathrm{C}$.

Sequence processing and analysis

SSU rRNA sequence data for 29 samples were provided by Gilberto Flores, CSU Northridge (Flores, unpublished data). Samples with a high percentage of archaeal reads were prioritized, including those with a high occurrence of recently proposed archaeal phyla, such as the Korarchaeota, Woesearchaeota, Pacearchaeota and Aigarchaeota.

DNA samples were sent to Oregon State University Center for Genome Research and sequenced using Illumina HiSeq 3000. A total of 1.2 billion paired-end reads ( 2 x150 bp) were generated (Table 5). Paired-end reads were trimmed for adapter sequences and quality using Trimmomatic (Bolger et al. 2014). The quality thresholds set discarded bases whose 4-base average fell below a Phred score of 20 and removed reads shorter 
than $50 \mathrm{bp}$.

\section{Community Composition}

The microbial composition of the environmental metagenomes were analyzed by extracting the SSU rRNA reads using SortMeRNA v2.0 and an e-value threshold of $10^{-5}$ (Kopylova et al. 2012). The reads were then classified using SINA v1.2.11 and a minimal 70\% identity threshold against the small subunit Ref NR 99123 database (Pruesse et al. 2012).

\section{Results}

From the ten metagenomes, 5.6 million SSU rRNA reads were classified. Around 4.9 million bacterial SSU rRNA reads were identified (Figure 12), while a total of 171,970 archaeal SSU rRNA reads were detected (Figure 13).

\section{Bacterial Composition}

Epsilonproteobacteria accounted for most of the classified SSU rRNA read counts in all samples, except at Vai Lili where the Gammaproteobacteria read counts were the most abundant. Epsilonproteobacteria only accounted for the majority (>50\%) of reads in five samples, all of the Mariner samples and Tui Malila-11. Furthermore, on average over $50 \%$ of the bacterial reads from each sample were classified to family level or below. At the family level, Nautiliaceae accounted for $24 \%$ of all read counts and was observed in all samples (Table 6). Campylobacteraceae, Hydrogenothermaceae and candidate division SR1 families accounted for $1.1 \%$ of total reads. Other candidate division bacterial lineages were identified such as Gracilibacteria (GN02) and Parcubacteria (OD1) which had relative abundances above $1 \%$ in several samples. 


\section{Archaeal Composition}

The most abundant archaeal lineages overall, based on classified SSU rRNA read counts were from Euryarchaeota (Figure 13). However, many uncultivated deep-sea hydrothermal vent Archaea were represented throughout the samples. The Woesearchaeota were present in all samples. At ABE-3, several uncultivated phyla were present at about $1 \%$ archaeal abundances. These were the Marine Hydrothermal Vent

Group, Marine Hydrothermal Vent Group 1, Bathyarchaeota (formally Miscellaneous Crenarchaeota Group) and Miscellaneous Euryarchaeota Group. Aenigmarchaeota were present in low abundance in all samples except Mariner-1. Nanoarchaeotal reads were detected in seven out of the ten samples and korarchaeotal sequences were detected in all samples (Table 5).

\section{Discussion}

In this study, metagenomic sequencing and SSU rRNA classification were used to characterize ten hydrothermal vent samples from four vent fields along the ELSC and VFR. Results were similar to previous microbial composition studies, which showed that Epsilonproteobacteria were the dominant lineage from the majority of hydrothermal vent systems (Hoek et al. 2003; Flores et al. 2011; Flores et al. 2012; Gulmann et al. 2015). In agreement with Flores et al. (2012), samples from the Mariner vent field were abundant in euryarchaeotal sequences, while at other vent locations, crenarchaeotal sequences were abundant. It is important to note that the short metagenomic reads (max. 150bp) are spread across any region of the SSU rRNA, potentially skewing classification efforts. This became apparent in the many sequences that could not be classified beyond the bacterial or archaeal domains, as well as the majority of Nautiliaceae reads that could not 
be further classified. In addition, the comparisons in this study were made with read counts, without trying to approximate SSU rRNA gene copy numbers for each lineage. This is more problematic in bacterial lineages and may cause them to be overrepresented in bacterial to archaeal comparisons.

\section{Bacterial Communities}

The most abundant bacterial communities were from Epsilonproteobacteria. Similar to the Flores et al. (2012) study, Helicobacteraceae, a mesophilic lineage, were more abundant in the northern vent fields, while Nautiliaceae, a thermophilic family, were more abundant in the Mariner vent field. Flores et al. (2012) did observe an unexpected abundance of Lebetimonas sequences (Flores et al. 2011), whereas in this study, very few Lebetimonas sequences were classified. The discrepancy is attributed to the few Nautiliaceae reads that were classified beyond the family level.

This study also highlights certain vent samples that could be targeted for genome recovery of uncultured bacterial lineages. Candidate division SR1, Gracilibacteria (GN02) and Parcubacteria (OD1) have not been cultured. Through metagenomic and single cell sequencing, draft genomes are available for these bacterial lineages (Campbell et al. 2013; Kantor et al. 2013; Rinke et al. 2013), but none have been recovered from the ELSC. Additionally, candidate division SR1 varied in abundance at Mariner-1 and Mariner-2, both subsamples from the same sulfide. These samples would be good candidates for differential coverage assembly and binning to recover a high quality SR1 draft genome.

\section{Archaeal Communities}

In this study, specific samples were selected based on archaeal diversity. Thus 
differences should be expected when compared to the microbial diversity study by Flores et al. (2012). The differences included higher abundances of Archaeoglobaceae, Korarchaeota and Methanocaldococcaceae, while there were lower abundances of Thermofilaceae and Nanoarchaeota.

Comparative differences between this study and Flores et al. (2012) were observed in many archaeal branches. In Flores et al. (2012), Thermofilaceae were prevalent in chimney communities in all sites, except Mariner. In the current samples, Thermofilaceae only accounted for a fraction of reads from ABE-1 and Tui Malila-10. Contrary to Flores et al. (2012), Archaeoglobaceae were a major part of the archaeal community in four of the ten sulfides. This is probably because of the known bias of the primers used by Flores et al. (2012) (Shakya et al. 2013). Two samples, Mariner-10 and Mariner-17, each accounted for greater than $60 \%$ of the archaeal reads. Nanoarchaeotal sequences accounted for only a small percentage of reads in seven of the sulfide samples (Table 5). Flores et al. (2012) showed several sulfide samples with relatively abundant nanoarchaeotal representation, but all were from the northern ELSC vent fields. A similar trend was observed for korarchaeotal sequences, which were detected in all ten samples. However, they were more abundant in northern vents fields, except for Via Lili-2. In 2005, fluid samples from Tui Malila had low concentrations of $\mathrm{H}_{2}$ (Mottl et al. 2011). In previous studies, low $\mathrm{H}_{2}$ concentration has correlated with a low presence of methanogens, so the high abundance of Methanocaldococcaceae sequences at Tui Malila-11 was unexpected (Flores et al. 2011; Flores et al. 2012a).

Crenarchaeotal and uncultured archaeal sequences were abundant in several 
sulfide samples. Assembly and binning of the ten metagenomes could reconstruct the metabolism of several of these uncultured deep-sea microbes. The metabolic reconstruction of ten hydrothermal vent sulfides will be the largest deep-sea hydrothermal vent microbial comparative study to date. The data once fully analyzed will likely provide a more comprehensive picture of the microbial processes within the vent fields at ELSC-VFR. 


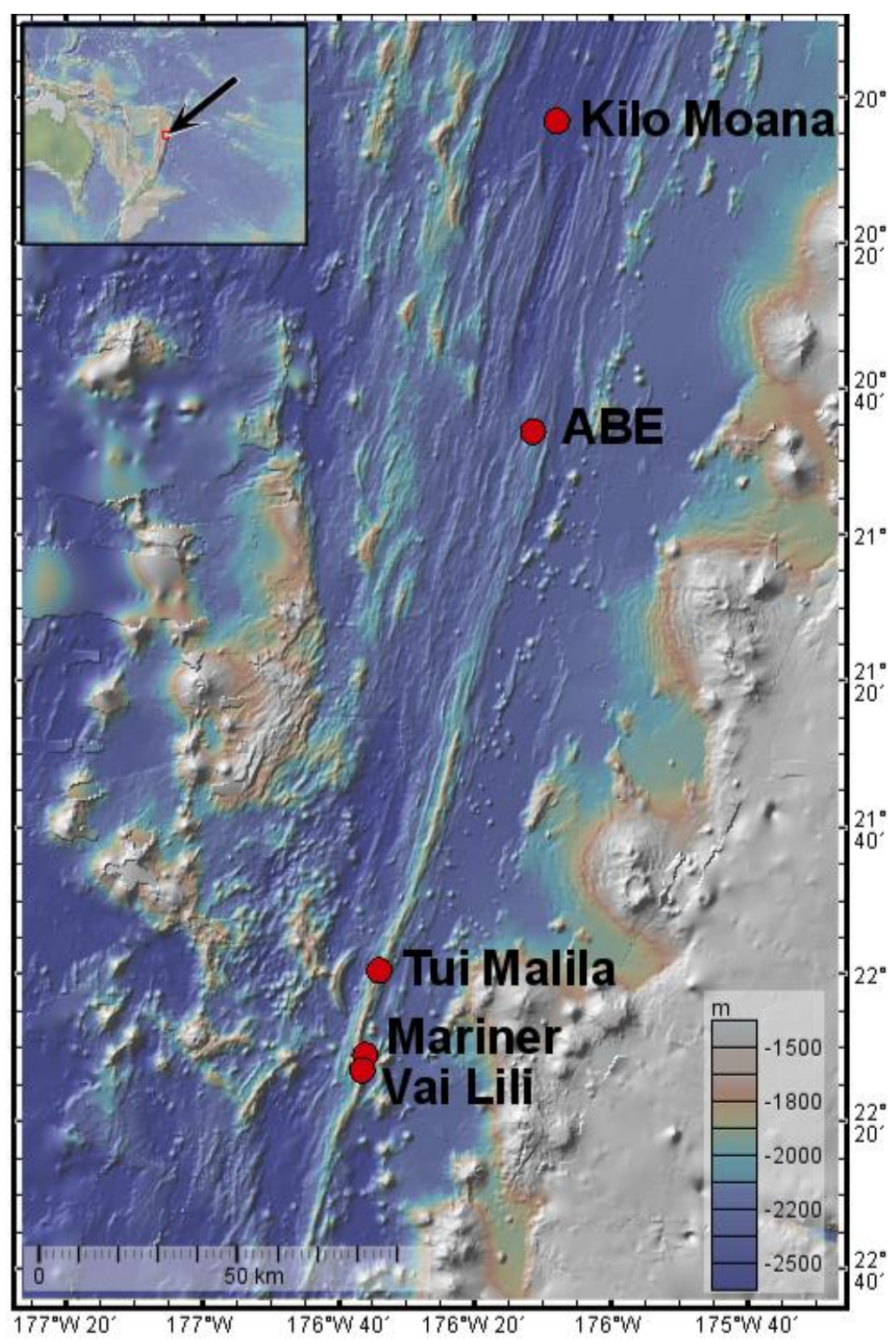

Figure 11. Hydrothermal vent fields along ELSC and VFR. Map was generated using GeoMapApp (http://www.geomapapp.org) 
Table 5. Raw sequence data, trimmed reads and SSU rRNA read counts by site. Listed are Nanoarchaeota and Korarchaeota read counts and percentage abundance per site.

\begin{tabular}{l|ccccc}
\hline & ABE-1 & ABE-3 & Mariner-1 & Mariner-2 & Vali Lili-2 \\
Raw Paired Reads & $412,295,856$ & $437,906,048$ & $294,978,076$ & $444,287,522$ & $310,302,926$ \\
\hline Avg. Read (bp) & 139.10 & 133.94 & 141.77 & 138.76 & 139.42 \\
Total bp (Gbp) & 43.5 & 40.4 & 35.5 & 49.1 & 34.0 \\
Reads Paired & $312,659,126$ & $301,881,238$ & $250,687,500$ & $353,874,178$ & $243,508,032$ \\
Reads Unpaired & $99,636,730$ & $136,024,810$ & $44,290,576$ & $90,413,344$ & $66,794,894$ \\
Percentage Lost & $24.17 \%$ & $31.06 \%$ & $15.01 \%$ & $20.35 \%$ & $21.53 \%$ \\
\hline Total SSU rRNA Reads & 449,275 & 501,224 & $1,315,104$ & 611,241 & 179,841 \\
Archaea SSU rRNA & 33,619 & 7,048 & 13,176 & 11,116 & 4,486 \\
Archaea (\%) & $7.48 \%$ & $1.41 \%$ & $1.00 \%$ & $1.82 \%$ & $2.49 \%$ \\
Nanoarchaeota (\#/\%) & $17 / 0.05 \%$ & $5 / 0.07 \%$ & $0 / 0.0 \%$ & $0 / 0.0 \%$ & $3 / 0.07 \%$ \\
Korarchaeota (\#/\%) & $3,305 / 9.83 \%$ & $155 / 2.20 \%$ & $3 / 0.02 \%$ & $8 / 0.07 \%$ & $1,220 / 27.0 \%$ \\
Bacteria SSU rRNA & 392,451 & 281,336 & $1,300,099$ & 569,538 & 169,913 \\
Bacteria (\%) & $87.35 \%$ & $56.13 \%$ & $98.86 \%$ & $93.18 \%$ & $94.48 \%$ \\
Eukarya SSU rRNA & 23,205 & 212,840 & 1,829 & 30,587 & 5,442 \\
Eukarya (\%) & $5.16 \%$ & $42.46 \%$ & $0.14 \%$ & $5.00 \%$ & $3.03 \%$ \\
\hline & Mariner-10 & Mariner-17 & Tui Malila-10 & Tui Malila-11 & Tui Malila-2 \\
Raw Paired Reads & $266,076,280$ & $331,585,098$ & $399,974,900$ & $226,203,648$ & $341,621,574$ \\
\hline Avg. Read (bp) & 138.38 & 134.94 & 134.29 & 133.79 & 134.50 \\
Total bp (Gbp) & 29.6 & 33.1 & 37.1 & 22.0 & 33.7 \\
Reads Paired & $214,104,898$ & $245,155,634$ & $276,069,690$ & $164,640,234$ & $250,390,060$ \\
Reads Unpaired & $51,971,382$ & $86,429,464$ & $123,905,210$ & $61,563,414$ & $91,231,514$ \\
Percentage Lost & $19.53 \%$ & $26.07 \%$ & $30.98 \%$ & $27.22 \%$ & $26.71 \%$ \\
\hline Total SSU rRNA Reads & 707,086 & 584,636 & 473,091 & 487,524 & 320,762 \\
Archaea SSU rRNA & 25,342 & 23,983 & 25,373 & 22,445 & 5,382 \\
Archaea (\%) & $3.58 \%$ & $4.10 \%$ & $5.36 \%$ & $4.60 \%$ & $1.68 \%$ \\
Nanoarchaeota (\#/\%) & $27 / 0.11 \%$ & $5 / 0.02 \%$ & $26 / 0.10 \%$ & $32 / 0.14 \%$ & - \\
Korarchaeota (\#/\%) & $2 / 0.01 \%$ & $9 / 0.04 \%$ & $602 / 2.37 \%$ & $30 / 0.13 \%$ & $100 / 1.86 \%$ \\
Bacteria SSU rRNA & 681,368 & 560,396 & 394,930 & 256,033 & 304,961 \\
Bacteria (\%) & 96.36 & $95.85 \%$ & $83.48 \%$ & $52.52 \%$ & $95.07 \%$ \\
Eukarya SSU rRNA & 376 & 257 & 52,788 & 209,046 & 10,419 \\
Eukarya (\%) & $0.05 \%$ & $0.04 \%$ & $11.16 \%$ & $42.88 \%$ & $3.25 \%$ \\
\hline
\end{tabular}


Table 6. Summary of epsilonproteobacterial abundances. Percentages are based on total read counts of epsilonproteobacterial SSU rRNA per sample. SSU rRNA reads were identified with SortMeRNA and classified with SINA against the SILVA SSU Ref NR123 database.

\begin{tabular}{|c|c|c|c|c|c|}
\hline & ABE-1 & ABE-3 & $\begin{array}{c}\text { Tui Malila- } \\
10 \\
\end{array}$ & $\begin{array}{c}\text { Tui Malila- } \\
11\end{array}$ & $\begin{array}{c}\text { Tui } \\
\text { Malila-2 } \\
\end{array}$ \\
\hline Total bacterial read counts & 392,451 & 281,336 & 394,930 & 256,033 & 304,961 \\
\hline Total \&-proteobacteria read counts & 124,747 & 68,597 & 158,533 & 140,736 & 105,467 \\
\hline Epsilonproteobacteria & $36.8 \%$ & $34.8 \%$ & $25.0 \%$ & $17.0 \%$ & $21.2 \%$ \\
\hline Campylobacterales & $4.2 \%$ & $6.8 \%$ & $6.3 \%$ & $6.7 \%$ & $6.7 \%$ \\
\hline Campylobacteraceae & $0.7 \%$ & $1.0 \%$ & $1.4 \%$ & $0.9 \%$ & $1.5 \%$ \\
\hline Arcobacter & $0.3 \%$ & $1.2 \%$ & $0.5 \%$ & $0.2 \%$ & $0.3 \%$ \\
\hline Campylobacter & $1.0 \%$ & $2.2 \%$ & $0.9 \%$ & $0.2 \%$ & $3.0 \%$ \\
\hline Sulfurospirillum & $0.2 \%$ & $0.5 \%$ & $0.2 \%$ & $0.1 \%$ & $0.6 \%$ \\
\hline Thiofractor & $0.0 \%$ & $0.0 \%$ & $0.0 \%$ & $0.0 \%$ & $0.0 \%$ \\
\hline Helicobacteraceae & $2.0 \%$ & $3.9 \%$ & $3.9 \%$ & $4.3 \%$ & $3.9 \%$ \\
\hline Helicobacter & $0.3 \%$ & $0.4 \%$ & $0.3 \%$ & $0.4 \%$ & $0.4 \%$ \\
\hline Sulfuricurvum & $0.3 \%$ & $0.3 \%$ & $0.7 \%$ & $1.4 \%$ & $0.2 \%$ \\
\hline Sulfurimonas & $0.5 \%$ & $3.9 \%$ & $3.7 \%$ & $1.0 \%$ & $9.6 \%$ \\
\hline Sulfurovum & $20.9 \%$ & $32.0 \%$ & $32.5 \%$ & $43.5 \%$ & $47.5 \%$ \\
\hline uncultured & $0.0 \%$ & $0.0 \%$ & $0.0 \%$ & $0.0 \%$ & $0.0 \%$ \\
\hline \multicolumn{6}{|l|}{ Nautiliales } \\
\hline Nautiliaceae & $28.7 \%$ & $10.0 \%$ & $22.3 \%$ & $22.8 \%$ & $3.9 \%$ \\
\hline Caminibacter & $0.4 \%$ & $0.1 \%$ & $0.3 \%$ & $0.3 \%$ & $0.0 \%$ \\
\hline Lebetimonas & $0.0 \%$ & $0.0 \%$ & $0.0 \%$ & $0.1 \%$ & $0.0 \%$ \\
\hline Nautilia & $0.0 \%$ & $0.0 \%$ & $0.0 \%$ & $0.0 \%$ & $0.0 \%$ \\
\hline Nitratifractor & $3.4 \%$ & $2.6 \%$ & $1.6 \%$ & $1.1 \%$ & $1.2 \%$ \\
\hline Nitratiruptor & $0.2 \%$ & $0.2 \%$ & $0.3 \%$ & $0.1 \%$ & $0.0 \%$ \\
\hline Thioreductor & $0.0 \%$ & $0.0 \%$ & $0.0 \%$ & $0.0 \%$ & $0.0 \%$ \\
\hline uncultured & $0.0 \%$ & $0.0 \%$ & $0.0 \%$ & $0.0 \%$ & $0.0 \%$ \\
\hline Total bacterial read counts & $1,300,099$ & 569,538 & 681,368 & 560,396 & 169,913 \\
\hline \multirow{2}{*}{ Total \&-proteobacteria read counts } & $\mathbf{1 , 0 5 0 , 3 3 4}$ & 336,730 & 566,716 & 338,927 & 19,688 \\
\hline & Mariner-1 & Mariner-2 & Mariner-10 & Mariner-17 & Via Lili-2 \\
\hline Epsilonproteobacteria & $8.6 \%$ & $15.6 \%$ & $32.7 \%$ & $24.2 \%$ & $24.2 \%$ \\
\hline Campylobacterales & $1.4 \%$ & $7.0 \%$ & $4.4 \%$ & $2.6 \%$ & $4.5 \%$ \\
\hline Campylobacteraceae & $0.3 \%$ & $1.4 \%$ & $1.6 \%$ & $0.6 \%$ & $0.5 \%$ \\
\hline Arcobacter & $0.1 \%$ & $0.4 \%$ & $0.2 \%$ & $0.1 \%$ & $0.2 \%$ \\
\hline Campylobacter & $0.0 \%$ & $0.1 \%$ & $1.1 \%$ & $0.8 \%$ & $1.8 \%$ \\
\hline Sulfurospirillum & $0.0 \%$ & $0.4 \%$ & $1.3 \%$ & $0.5 \%$ & $0.6 \%$ \\
\hline
\end{tabular}




\begin{tabular}{rccccc} 
Thiofractor & $0.0 \%$ & $0.1 \%$ & $0.0 \%$ & $0.0 \%$ & $0.0 \%$ \\
\hline Helicobacteraceae & & $10.5 \%$ & $1.4 \%$ & $3.1 \%$ & $3.1 \%$ \\
Helicobacter & $0.1 \%$ & $0.2 \%$ & $0.3 \%$ & $0.3 \%$ & $0.5 \%$ \\
Sulfuricurvum & $0.0 \%$ & $0.1 \%$ & $0.1 \%$ & $0.1 \%$ & $0.1 \%$ \\
Sulfurimonas & $9.2 \%$ & $39.1 \%$ & $4.8 \%$ & $8.1 \%$ & $4.6 \%$ \\
Sulfurovum & $0.6 \%$ & $13.2 \%$ & $9.2 \%$ & $21.2 \%$ & $53.1 \%$ \\
uncultured & $0.0 \%$ & $0.0 \%$ & $0.0 \%$ & $0.0 \%$ & $0.0 \%$ \\
\hline Nautiliales & & & & & \\
Nautiliaceae & & $10.8 \%$ & $39.8 \%$ & $36.2 \%$ & $4.5 \%$ \\
Caminibacter & $1.0 \%$ & $0.1 \%$ & $0.2 \%$ & $0.3 \%$ & $0.0 \%$ \\
Lebetimonas & $0.0 \%$ & $0.1 \%$ & $0.1 \%$ & $0.3 \%$ & $0.0 \%$ \\
Nautilia & $0.0 \%$ & $0.0 \%$ & $0.0 \%$ & $0.0 \%$ & $0.0 \%$ \\
Nitratifractor & $0.1 \%$ & $1.0 \%$ & $2.8 \%$ & $1.6 \%$ & $2.4 \%$ \\
Nitratiruptor & $0.0 \%$ & $0.0 \%$ & $0.0 \%$ & $0.0 \%$ & $0.0 \%$ \\
Thioreductor & $0.0 \%$ & $0.0 \%$ & $0.0 \%$ & $0.0 \%$ & $0.0 \%$ \\
uncultured & $0.1 \%$ & $0.0 \%$ & $0.0 \%$ & $0.0 \%$ & $0.0 \%$ \\
\hline
\end{tabular}




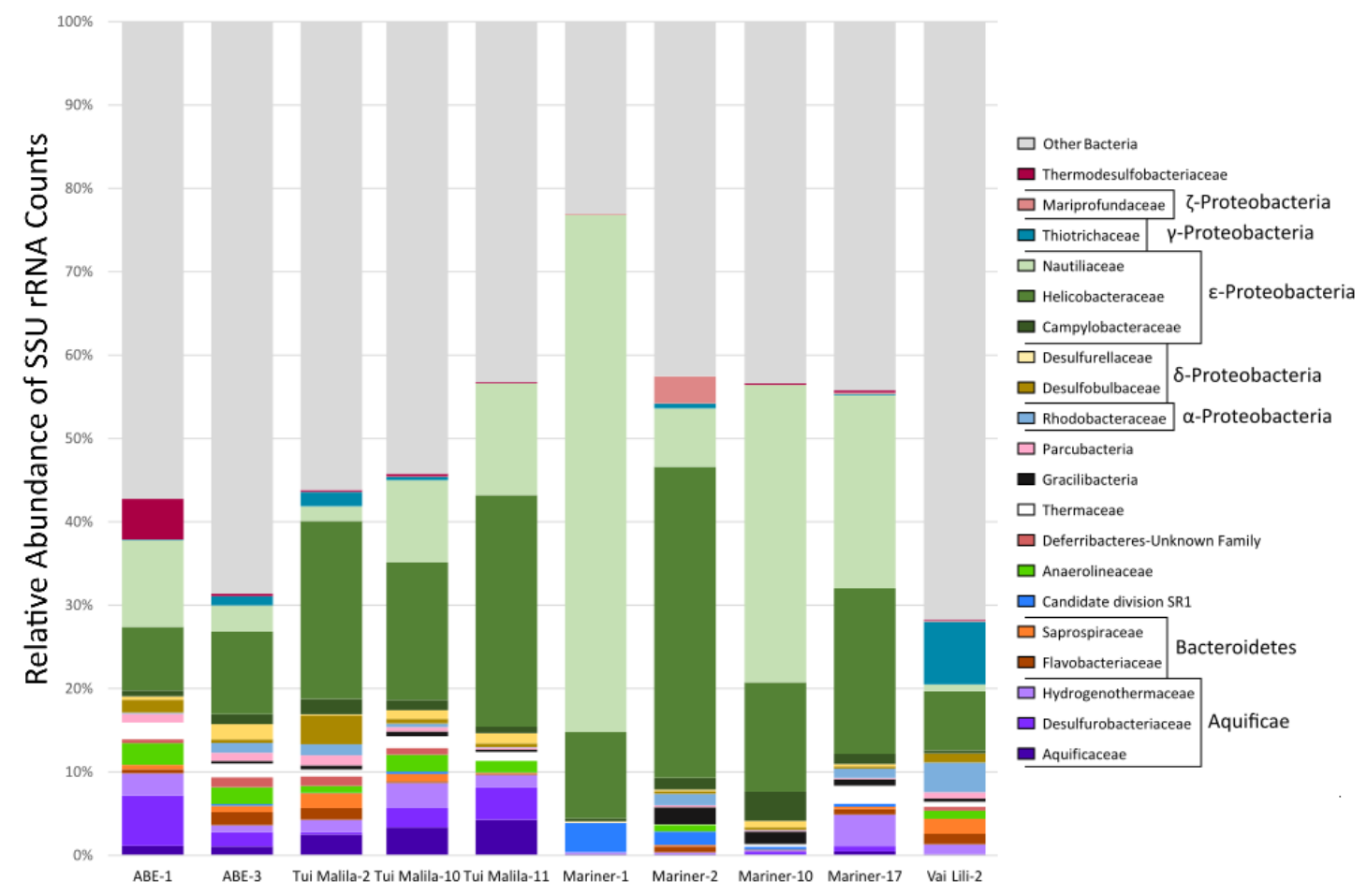

Figure 12. Relative abundance of bacterial families from sulfide samples taken along the ELSC and VFR. Only bacterial families with greater than $1 \%$ abundance are shown. All other bacteria are grouped together under "Other Bacteria". SSU rRNA reads were identified with SortMeRNA and classified with SINA against the SILVA SSU Ref NR123 database. 


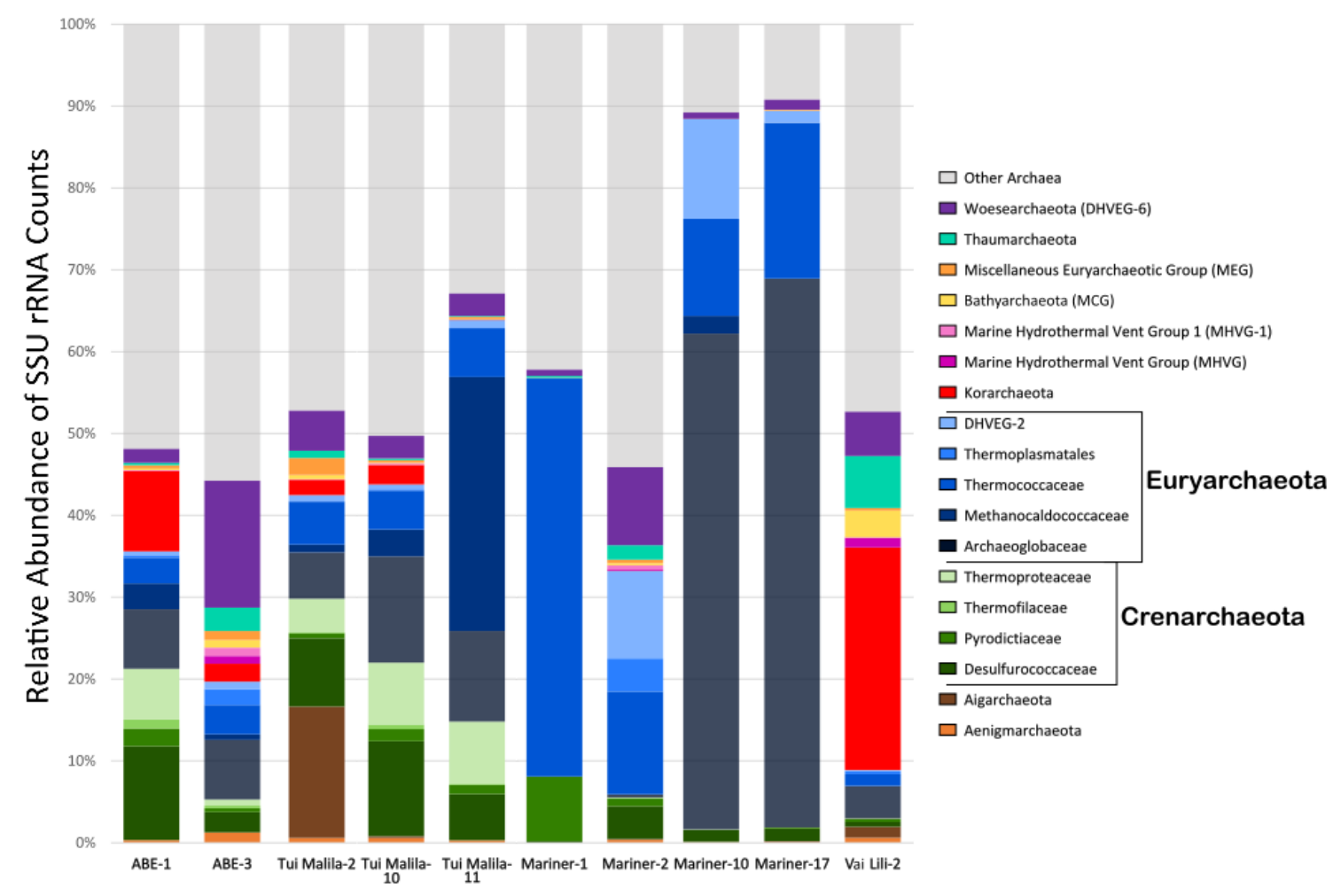

Figure 13. Relative abundance of archaeal families from sulfide samples taken along the ELSC and VFR. Only archaeal families with greater than $1 \%$ abundance are shown. All other Archaea are grouped together under Other Archaea. SSU rRNA reads were identified with SortMeRNA and classified with SINA against the SILVA SSU Ref NR123 database. 


\section{Chapter 4: Conclusion}

Metagenomics has become a revolutionary tool for microbial ecologists.

Advanced techniques, such as differential coverage, have led to the successful recovery

of low abundant microbes. Metagenomics is also useful in profiling community composition without primer biases; potentially revealing a more accurate picture of the community diversity and identifying rare microbes missed using traditional cultivated independent and dependent methods.

In Chapter 2, differential coverage binning was used to recover two archaeal genomes from low abundant samples from a New Zealand hot spring. The recovered korarchaeotal and nanoarchaeotal genomes were the first representative genomes from New Zealand. Both archaeal genomes show extensive genomic synteny between their respective terrestrial lineages. The enrichment of crenarchaeotal sequences in the NZ13 cultures show a continued pattern of crenarchaeotes being the only known nanoarchaeotal host. The host metabolisms, in particular the presence or absence of archaella, correlate with the retention or reduction of nanoarchaeotal archaellum genes. Further suggesting the absence of archaella in the New Zealand host. Though host physiology does not depict whether or not the nanoarchaeotes retain gluconeogenesis/glycolysis genes. The host of Nst1 is suspected to be an autotroph, while the host of N. acidilobi is a heterotroph. Seemingly unrelated to the host physiology, the NZ13 nanoarchaeote contains CRISPR type I-B genes, which are not present in the other representative terrestrial nanoarchaeotes. The marine nanoarchaeote, Nanoarchaeum equitans, contains the same CRISPR type I-B gene. This supports a previous conclusion, that the CRISPR system has been lost through genome reduction in the Yellowstone National Park (YNP) 
nanoarchaeotes. The genome reduction process likely occurred after the YNP nanoarchaeotes and NZ13 nanoarchaeote lineages diverged (Randau 2012; Podar et al. 2013). The next step will be to identify the NZ13 nanoarchaeote host. So far all nanoarchaeotes isolated or sequenced have hosts from different crenarchaeotal orders (Huber et al. 2002; Podar et al. 2013; Wurch et al. 2016). More nanoarchaeote-host systems may help resolve the underlying mechanisms to the nanoarchaeotes attachment, genome reduction process, host specificity and nutrient transfer.

In addition, the NZ13 nanoarchaeote and NZ13 korarchaeote genomes possess osmotic regulation genes which are unique to the New Zealand genomes. The uptake of potassium may provide the organisms a higher salt tolerance. The osmotic regulation genes were the only differences among the three korarchaeotal genomes compared in this study. Major differences were observed between the terrestrial and marine Nanoarchaeota lineages, therefore a marine korarchaeotal genome will provide insights into the conserved korarchaeotal physiologies.

In Chapter 3, metagenomic sequencing and SSU rRNA identification tools were used to classify and compare ten microbial communities from ELSC-VFR. The community profiles showed Epsilonproteobacteria were the most abundant microbes at hydrothermal vents, with the exception of Vai Lili. The Euryarchaeota were the most abundant at the Mariner vent locations, while the Crenarchaeota were more abundant at $\mathrm{ABE}$ and Tui Malila. Korarchaeotal sequences were detected in relatively high abundance in five samples. A korarchaeotal genome should easily be recovered from the ELSC metagenomes and provide the first genome of a marine korarchaeote. In contrast, 
the Nanoarchaeota were present in such low numbers, assembling a near complete genome may be difficult. Other genomes from uncultured members of both the bacterial and archaeal should be recoverable. 


\section{References:}

Albertsen, M., Hugenholtz, P., Skarshewski, A., Nielsen, K.L., Tyson, G.W. and Nielsen, P.H., 2013. Genome sequences of rare, uncultured bacteria obtained by differential coverage binning of multiple metagenomes. Nature Biotechnology, 31(6), pp.533-8.

Aoki-Kinoshita, K.F. and Kanehisa, M., 2007. Gene annotation and pathway mapping in KEGG. Methods In Molecular Biology, 396, pp.71-91.

Auchtung, T.A., Takacs-Vesbach, C.D. and Cavanaugh, C.M., 2006. 16S rRNA phylogenetic investigation of the candidate division "Korarchaeota." Applied and Environmental Microbiology, 72(7), pp.5077-5082.

Auguet, J.-C., Barberan, A. and Casamayor, E.O., 2010. Global ecological patterns in uncultured Archaea. The ISME Journal, 4(2), pp.182-90.

Baker, B.J., Comolli, L.R., Dick, G.J., Hauser, L.J., Hyatt, D., Dill, B.D., Land, M.L., Verberkmoes, N.C., Hettich, R.L. and Banfield, J.F., 2010. Enigmatic, ultrasmall, uncultivated Archaea. Proceedings of the National Academy of Sciences of the United States of America, 107(19), pp.8806-11.

Baker, B.J., Saw, J.H., Lind, A.E., Lazar, C.S., Hinrichs, K., Teske, A.P. and Ettema, T.J.G., 2016. Genomic inference of the metabolism of cosmopolitan subsurface Archaea, Hadesarchaea. Nature Microbiology, 1(3), p.16002.

Barns, S.M., Delwiche, C.F., Palmer, J.D. and Pace, N.R., 1996. Perspectives on archaeal diversity, thermophily and monophyly from environmental rRNA sequences. Proceedings of the National Academy of Sciences of the United States of America, 93(17), pp.9188-93.

Barns, S.M., Fundyga, R.E., Jeffries, M.W. and Pace, N.R., 1994. Remarkable archaeal 
diversity detected in a Yellowstone National Park hot spring environment. Proceedings of the National Academy of Sciences of the United States of America, 91(5), pp.1609-1613.

Béjà, O., Aravind, L., Koonin, E. V, Suzuki, M.T., Hadd, A., Nguyen, L.P., Jovanovich, S.B., Gates, C.M., Feldman, R.A., Spudich, J.L., Spudich, E.N. and DeLong, E.F., 2000. Bacterial rhodopsin: evidence for a new type of phototrophy in the sea. Science, 289(5486), pp.1902-6.

Bolger, A.M., Lohse, M. and Usadel, B., 2014. Trimmomatic: a flexible trimmer for Illumina sequence data. Bioinformatics, 30(15), pp.2114-2120.

Bomar, L., Maltz, M., Colston, S. and Graf, J., 2011. Directed culturing of microorganisms using metatranscriptomics. mBio, 2(2), pp.1-8.

Brochier-Armanet, C., Boussau, B., Gribaldo, S. and Forterre, P., 2008. Mesophilic Crenarchaeota: proposal for a third archaeal phylum, the Thaumarchaeota. Nature Reviews Microbiology, 6(3), pp.245-252.

Camacho, C., Coulouris, G., Avagyan, V., Ma, N., Papadopoulos, J., Bealer, K. and Madden, T.L., 2009. BLAST+: architecture and applications. BMC Bioinformatics, 10(421), p.421.

Campbell, J.H., O’Donoghue, P., Campbell, A.G., Schwientek, P., Sczyrba, A., Woyke, T., Söll, D. and Podar, M., 2013. UGA is an additional glycine codon in uncultured SR1 bacteria from the human microbiota. Proceedings of the National Academy of Sciences of the United States of America, 110(14), pp.5540-5.

Casanueva, A., Galada, N., Baker, G.C., Grant, W.D., Heaphy, S., Jones, B., Yanhe, M., 
Ventosa, A., Blamey, J. and Cowan, D. a., 2008. Nanoarchaeal 16S rRNA gene sequences are widely dispersed in hyperthermophilic and mesophilic halophilic environments. Extremophiles, 12(5), pp.651-656.

Castelle, C.J., Wrighton, K.C., Thomas, B.C., Hug, L.A., Brown, C.T., Wilkins, M.J., Frischkorn, K.R., Tringe, S.G., Singh, A., Markillie, L.M., Taylor, R.C., Williams, K.H. and Banfield, J.F., 2015. Genomic expansion of domain Archaea highlights roles for organisms from new phyla in anaerobic carbon cycling. Current Biology, 25(6), pp.690-701.

Cheong, W.H., Tan, Y.C., Yap, S.J. and Ng, K.P., 2015. ClicO FS: An interactive webbased service of Circos. Bioinformatics, 31(22), pp.3685-3687.

Corre, E., 2001. $\varepsilon$-Proteobacterial diversity from a deep-sea hydrothermal vent on the Mid-Atlantic Ridge. FEMS Microbiology Letters, 205(2), pp.329-335.

Costa, K.C., Wong, P.M., Wang, T.S., Lie, T.J., Dodsworth, J.A., Swanson, I., Burn, J.A., Hackett, M. and Leigh, J.A., 2010. Protein complexing in a methanogen suggests electron bifurcation and electron delivery from formate to heterodisulfide reductase. Proceedings of the National Academy of Sciences of the United States of America, 107(24), pp.11050-11055.

Eddy, S.R., 1998. Profile hidden Markov models. Bioinformatics, 14(9), pp.755-63.

Elkins, J.G., Podar, M., Graham, D.E., Makarova, K.S., Wolf, Y., Randau, L., Hedlund, B.P., Brochier-Armanet, C., Kunin, V., Anderson, I., Lapidus, A., Goltsman, E., Barry, K., Koonin, E. V, Hugenholtz, P., Kyrpides, N., Wanner, G., Richardson, P., Keller, M., et al., 2008. A korarchaeal genome reveals insights into the evolution of 
the Archaea. Proceedings of the National Academy of Sciences of the United States of America, 105(23), pp.8102-8107.

Erauso, G., Reysenbach, A.-L., Godfroy, A., Meunier, J.-R., Crump, B., Partensky, F., Baross, J., Marteinsson, V., Barbier, G., Pace, N. and Prieur, D., 1993. Pyrococcus abyssi $\mathrm{sp}$. nov., a new hyperthermophilic archaeon isolated from a deep-sea hydrothermal vent. Archives of Microbiology, 160(5), pp.307-10.

Ferguson, T.J. and Mah, R.A., 1983. Effect of $\mathrm{H}(2)-\mathrm{CO}(2)$ on methanogenesis from acetate or methanol in Methanosarcina spp. Applied and Environmental Microbiology, 46(2), pp.348-355.

Ferrini, V.L., Tivey, M.K., Carbotte, S.M., Martinez, F. and Roman, C., 2008. Variable morphologic expression of volcanic, tectonic, and hydrothermal processes at six hydrothermal vent fields in the Lau back-arc basin. Geochemistry, Geophysics, Geosystems, 9(7).

Flores, G.E., Campbell, J.H., Kirshtein, J.D., Meneghin, J., Podar, M., Steinberg, J.I., Seewald, J.S., Tivey, M.K., Voytek, M. a, Yang, Z.K. and Reysenbach, A.-L., 2011. Microbial community structure of hydrothermal deposits from geochemically different vent fields along the Mid-Atlantic Ridge. Environmental Microbiology, 13(8), pp.2158-71.

Flores, G.E., Shakya, M., Meneghin, J., Yang, Z.K., Seewald, J.S., Geoff Wheat, C., Podar, M. and Reysenbach, A.-L., 2012a. Inter-field variability in the microbial communities of hydrothermal vent deposits from a back-arc basin. Geobiology, 10(4), pp.333-46. 
Flores, G.E., Wagner, I.D., Liu, Y. and Reysenbach, A.-L., 2012b. Distribution, abundance, and diversity patterns of the thermoacidophilic "deep-sea hydrothermal vent euryarchaeota 2". Frontiers in Microbiology, 3(February), p.47.

Fox, G.E., Magrum, L.J., Balch, W.E., Wolfe, R.S. and Woese, C.R., 1977. Classification of methanogenic bacteria by $16 \mathrm{~S}$ ribosomal RNA characterization. Proceedings of the National Academy of Sciences of the United States of America, 74(10), pp.453741.

Giovannoni, S.J., Britschgi, T.B., Moyer, C.L. and Field, K.G., 1990. Genetic diversity in Sargasso Sea bacterioplankton. Nature, 345(6270), pp.60-3.

Giuffrè, A., Borisov, V.B., Arese, M., Sarti, P. and Forte, E., 2014. Cytochrome bd oxidase and bacterial tolerance to oxidative and nitrosative stress. Biochimica et Biophysica Acta (BBA) - Bioenergetics, 1837(7), pp.1178-1187.

González, J.M., Masuchi, Y., Robb, F.T., Ammerman, J.W., Maeder, D.L., Yanagibayashi, M., Tamaoka, J. and Kato, C., 1998. Pyrococcus horikoshii sp. nov., a hyperthermophilic archaeon isolated from a hydrothermal vent at the Okinawa Trough. Extremophiles, 2(2), pp.123-130.

Griffiths-Jones, S., Bateman, A., Marshall, M., Khanna, A. and Eddy, S.R., 2003. Rfam: An RNA family database. Nucleic Acids Research, 31(1), pp.439-441.

Gulmann, L.K., Beaulieu, S.E., Shank, T.M., Ding, K., Seyfried, W.E. and Sievert, S.M., 2015. Bacterial diversity and successional patterns during biofilm formation on freshly exposed basalt surfaces at diffuse-flow deep-sea vents. Frontiers in Microbiology, 6(September), pp.1-16. 
Haft, D.H., Selengut, J.D. and White, O., 2003. The TIGRFAMs database of protein families. Nucleic Acids Research, 31(1), pp.371-3.

Handelsman, J., Rondon, M.R., Brady, S.F., Clardy, J. and Goodman, R.M., 1998. Molecular biological access to the chemistry of unknown soil microbes: a new frontier for natural products. Chemistry \& Biology, 5(10), pp.R245-R249.

Hoek, J., Banta, A., Hubler, F. and Reysenbach, A.-L., 2003. Microbial diversity of a sulphide spire located in the Edmond deep-sea hydrothermal vent field on the Central Indian Ridge. Geobiology, 1(2), pp.119-127.

Huber, H., Burggraf, S., Mayer, T., Wyschkony, I., Rachel, R. and Stetter, K.O., 2000. Ignicoccus gen. nov., a novel genus of hyperthermophilic, chemolithoautotrophic Archaea, represented by two new species, Ignicoccus islandicus sp. nov. and Ignicoccus pacificus sp. nov. International Journal of Systematic and Evolutionary Microbiology, 50(6), pp.2093-2100.

Huber, H., Hohn, M.J., Rachel, R., Fuchs, T., Wimmer, V.C. and Stetter, K.O., 2002. A new phylum of Archaea represented by a nanosized hyperthermophilic symbiont. Nature, 417(6884), pp.63-7.

Hug, L.A., Baker, B.J., Anantharaman, K., Brown, C.T., Probst, A.J., Castelle, C.J., Butterfield, C.N., Hernsdorf, A.W., Amano, Y., Ise, K., Suzuki, Y., Dudek, N., Relman, D.A., Finstad, K.M., Amundson, R., Thomas, B.C. and Banfield, J.F., 2016. A new view of the tree of life. Nature Microbiology, 1(5), p.16048.

Huson, D.H., Beier, S., Flade, I., Górska, A., El-Hadidi, M., Mitra, S., Ruscheweyh, H.-J. and Tappu, R., 2016. MEGAN community edition-interactive exploration and 
analysis of large-scale microbiome sequencing data. PLoS Computational Biology, 12(6), p.e1004957.

Hyatt, D., Chen, G.-L., LoCascio, P.F., Land, M.L., Larimer, F.W. and Hauser, L.J., 2010. Prodigal: prokaryotic gene recognition and translation initiation site identification. BMC Bioinformatics, 11(1), p.119.

Imelfort, M., Parks, D., Woodcroft, B.J., Dennis, P., Hugenholtz, P. and Tyson, G.W., 2014. GroopM: an automated tool for the recovery of population genomes from related metagenomes. PeerJ, 2, p.e603.

Jahn, U., Gallenberger, M., Paper, W., Junglas, B., Eisenreich, W., Stetter, K.O., Rachel, R. and Huber, H., 2008. Nanoarchaeum equitans and Ignicoccus hospitalis: new insights into a unique, intimate association of two archaea. Journal of Bacteriology, 190(5), pp.1743-1750.

Jarrell, K.F. and Albers, S.V., 2012. The archaellum: An old motility structure with a new name. Trends in Microbiology, 20(7), pp.307-312.

Jolivet, E., L'Haridon, S., Corre, E., Forterre, P. and Prieur, D., 2003. Thermococcus gammatolerans sp. nov., a hyperthermophilic archaeon from a deep-sea hydrothermal vent that resists ionizing radiation. International Journal of Systematic and Evolutionary Microbiology, 53(Pt 3), pp.847-851.

Jones, P., Binns, D., Chang, H.Y., Fraser, M., Li, W., McAnulla, C., McWilliam, H., Maslen, J., Mitchell, A., Nuka, G., Pesseat, S., Quinn, A.F., Sangrador-Vegas, A., Scheremetjew, M., Yong, S.Y., Lopez, R. and Hunter, S., 2014. InterProScan 5: genome-scale protein function classification. Bioinformatics, 30(9), pp.1236-1240. 
Kantor, R.S., Wrighton, K.C., Handley, K.M., Sharon, I., Hug, L. a., Castelle, C.J., Thomas, B.C. and Banfield, J.F., 2013. Small genomes and sparse metabolisms of sediment-associated bacteria from four candidate phyla. mBio, 4(5), pp.e00708-13.

Kearse, M., Moir, R., Wilson, A., Stones-Havas, S., Cheung, M., Sturrock, S., Buxton, S., Cooper, A., Markowitz, S., Duran, C., Thierer, T., Ashton, B., Meintjes, P. and Drummond, A., 2012. Geneious Basic: An integrated and extendable desktop software platform for the organization and analysis of sequence data.

Bioinformatics, 28(12), pp.1647-1649.

Könneke, M., Bernhard, A.E., de la Torre, J.R., Walker, C.B., Waterbury, J.B. and Stahl, D. a, 2005. Isolation of an autotrophic ammonia-oxidizing marine archaeon. Nature, 437(7058), pp.543-6.

Kopylova, E., Noé, L. and Touzet, H., 2012. SortMeRNA: fast and accurate filtering of ribosomal RNAs in metatranscriptomic data. Bioinformatics, 28(24), pp.3211-7.

Kraegeloh, A., Amendt, B. and Kunte, H.J., 2005. Potassium transport in a halophilic member of the Bacteria domain: Identification and characterization of the $\mathrm{K}+$ uptake systems TrkH and TrkI from Halomonas elongata DSM 2581T. Journal of Bacteriology, 187(3), pp.1036-1043.

Krzywinski, M., Schein, J., Birol, I., Connors, J., Gascoyne, R., Horsman, D., Jones, S.J. and Marra, M.A., 2009. Circos: an information aesthetic for comparative genomics. Genome research, 19(9), pp.1639-45.

Langmead, B. and Salzberg, S.L., 2012. Fast gapped-read alignment with Bowtie 2. Nature Methods, 9(4), pp.357-359. 
Lee, S.H., Kim, M.-S., Kim, Y.J., Kim, T.W., Kang, S.G. and Lee, H.S., 2017. Transcriptomic profiling and its implications for the $\mathrm{H} 2$ production of a nonmethanogen deficient in the frhAGB-encoding hydrogenase. Applied Microbiology and Biotechnology, pp.1-8.

Li, H., Handsaker, B., Wysoker, A., Fennell, T., Ruan, J., Homer, N., Marth, G., Abecasis, G. and Durbin, R., 2009. The sequence alignment/map format and SAMtools. Bioinformatics, 25(16), pp.2078-2079.

Makarova, K.S., Wolf, Y.I., Alkhnbashi, O.S., Costa, F., Shah, S.A., Saunders, S.J., Barrangou, R., Brouns, S.J.J., Charpentier, E., Haft, D.H., Horvath, P., Moineau, S., Mojica, F.J.M., Terns, R.M., Terns, M.P., White, M.F., Yakunin, A.F., Garrett, R.A., van der Oost, J., et al., 2015a. An updated evolutionary classification of CRISPR-Cas systems. Nature Reviews Microbiology, 13(11), pp.722-736.

Makarova, K.S., Wolf, Y.I. and Koonin, E. V, 2015b. Archaeal Clusters of Orthologous Genes (arCOGs): an update and application for analysis of shared features between Thermococcales, Methanococcales, and Methanobacteriales. Life, 5(1), pp.818-40. Mardanov, A. V., Svetlitchnyi, V.A., Beletsky, A. V., Prokofeva, M.I., BonchOsmolovskaya, E.A., Ravin, N. V. and Skryabin, K.G., 2010. The genome sequence of the crenarchaeon Acidilobus saccharovorans supports a new order, Acidilobales, and suggests an important ecological role in terrestrial acidic hot springs. Applied and Environmental Microbiology, 76(16), pp.5652-5657.

Martin, D.D., Ciulla, R.A. and Roberts, M.F., 1999. Osmoadaptation in archaea. Applied and Environmental Microbiology, 65(5), pp.1815-1825. 
Martinez, F., Okino, K., Ohara, Y., Reysenbach, A.-L. and Goffredi, S., 2007. Back-Arc Basins. Oceanography, 20(1), pp.116-127.

Miller-Coleman, R.L., Dodsworth, J. a., Ross, C. a., Shock, E.L., Williams, A.J., Hartnett, H.E., McDonald, A.I., Havig, J.R. and Hedlund, B.P., 2012. Korarchaeota diversity, biogeography, and abundance in Yellowstone and Great Basin hot springs and ecological niche modeling based on machine learning. PLoS ONE, 7(5).

Mottl, M.J., Seewald, J.S., Wheat, C.G., Tivey, M.K., Michael, P.J., Proskurowski, G., McCollom, T.M., Reeves, E., Sharkey, J., You, C.-F., Chan, L.-H. and Pichler, T., 2011. Chemistry of hot springs along the Eastern Lau Spreading Center. Geochimica et Cosmochimica Acta, 75(4), pp.1013-1038.

Nercessian, O., Reysenbach, A.-L., Prieur, D. and Jeanthon, C., 2003. Archaeal diversity associated with in situ samplers deployed on hydrothermal vents on the East Pacific Rise (13oN). Environmental Microbiology, 5(6), pp.492-502.

Paper, W., Jahn, U., Hohn, M.J., Kronner, M., Näther, D.J., Burghardt, T., Rachel, R., Stetter, K.O. and Huber, H., 2007. Ignicoccus hospitalis sp. nov., the host of Nanoarchaeum equitans. International Journal of Systematic and Evolutionary Microbiology, 57(4), pp.803-808.

Parks, D.H., Imelfort, M., Skennerton, C.T., Hugenholtz, P. and Tyson, G.W., 2015. CheckM: assessing the quality of microbial genomes recovered from isolates, single cells, and metagenomes. Genome Research, 25(7), pp.1043-55.

Peng, Y., Leung, H.C.M., Yiu, S.M. and Chin, F.Y.L., 2012. IDBA-UD: a de novo assembler for single-cell and metagenomic sequencing data with highly uneven 
depth. Bioinformatics, 28(11), pp.1420-8.

Podar, M., Anderson, I., Makarova, K.S., Elkins, J.G., Ivanova, N., Wall, M.A., Lykidis, A., Mavromatis, K., Sun, H., Hudson, M.E., Chen, W., Deciu, C., Hutchison, D., Eads, J.R., Anderson, A., Fernandes, F., Szeto, E., Lapidus, A., Kyrpides, N.C., et al., 2008. A genomic analysis of the archaeal system Ignicoccus hospitalisNanoarchaeum equitans. Genome Biology, 9(11), p.R158.

Podar, M., Makarova, K.S., Graham, D.E., Wolf, Y.I., Koonin, E. V and Reysenbach, A.L., 2013. Insights into archaeal evolution and symbiosis from the genomes of a nanoarchaeon and its inferred crenarchaeal host from Obsidian Pool, Yellowstone National Park. Biology Direct, 8(1), p.9.

Prokofeva, M.I., Miroshnichenko, M.L., Kostrikina, N.A., Chernyh, N.A., Kuznetsov, B.B., Tourova, T.P. and Bonch-Osmolovskaya, E.A., 2000. Acidilobus aceticus gen. nov., s.p. nov., a novel anaerobic thermoacidophilic archaeon from continental hot vents in Kamchatka. International Journal of Systematic and Evolutionary Microbiology, 50(6), pp.2001-2008.

Pruesse, E., Peplies, J. and Glöckner, F.O., 2012. SINA: accurate high-throughput multiple sequence alignment of ribosomal RNA genes. Bioinformatics, 28(14), pp.1823-9.

Punta, M., Coggill, P.C., Eberhardt, R.Y., Mistry, J., Tate, J., Boursnell, C., Pang, N., Forslund, K., Ceric, G., Clements, J., Heger, A., Holm, L., Sonnhammer, E.L.L., Eddy, S.R., Bateman, A. and Finn, R.D., 2012. The Pfam protein families database. Nucleic Acids Research, 40(Database issue), pp.D290-301. 
Randau, L., 2012. RNA processing in the minimal organism Nanoarchaeum equitans. Genome biology, 13(7), p.R63.

Randau, L., Münch, R., Hohn, M.J., Jahn, D. and Söll, D., 2005. Nanoarchaeum equitans creates functional tRNAs from separate genes for their 5'- and 3'-halves. Nature, 433(1999), pp.537-541.

Reysenbach, A.-L., Liu, Y., Banta, A.B., Beveridge, T.J., Kirshtein, J.D., Schouten, S., Tivey, M.K., Von Damm, K.L. and Voytek, M. a, 2006. A ubiquitous thermoacidophilic archaeon from deep-sea hydrothermal vents. Nature, 442(7101), pp.444-7.

Reysenbach, A.-L., Longnecker, K. and Kirshtein, J., 2000. Novel bacterial and archaeal lineages from an in situ growth chamber deployed at a Mid-Atlantic Ridge hydrothermal vent. Applied and Environmental Microbiology, 66(9), pp.3798-3806.

Rinke, C., Schwientek, P., Sczyrba, A., Ivanova, N.N., Anderson, I.J., Cheng, J.-F., Darling, A.E., Malfatti, S., Swan, B.K., Gies, E. a, Dodsworth, J. a, Hedlund, B.P., Tsiamis, G., Sievert, S.M., Liu, W.-T., Eisen, J. a, Hallam, S.J., Kyrpides, N.C., Stepanauskas, R., et al., 2013. Insights into the phylogeny and coding potential of microbial dark matter. Nature, 499(7459), pp.431-437.

Saw, J.H., Spang, A., Zaremba-Niedzwiedzka, K., Juzokaite, L., Dodsworth, J.A., Murugapiran, S.K., Colman, D.R., Takacs-Vesbach, C., Hedlund, B.P., Guy, L. and Ettema, T.J.G., 2015. Exploring microbial dark matter to resolve the deep archaeal ancestry of eukaryotes. Philosophical Transactions of the Royal Society B:

Biological Sciences, 370(1678), p.20140328. 
Schmidt, A., Rzanny, M., Schmidt, A., Hagen, M., Schütze, E. and Kothe, E., 2012. GC content-independent amino acid patterns in Bacteria and Archaea. Journal of Basic Microbiology, 52(2), pp.195-205.

Shakya, M., Quince, C., Campbell, J.H., Yang, Z.K., Schadt, C.W. and Podar, M., 2013. Comparative metagenomic and rRNA microbial diversity characterization using archaeal and bacterial synthetic communities. Environmental microbiology, 15(6), pp.1882-99.

Southworth, T.W., Guffanti, A.A., Moir, A. and Krulwich, T.A., 2001. GerN, an endospore germination protein of Bacillus cereus, is an $\mathrm{Na}+\mathrm{H}+\mathrm{K}+$ antiporter. Journal of Bacteriology, 183(20), pp.5896-5903.

Spang, A., Saw, J.H., Jørgensen, S.L., Zaremba-Niedzwiedzka, K., Martijn, J., Lind, A.E., van Eijk, R., Schleper, C., Guy, L. and Ettema, T.J.G., 2015. Complex archaea that bridge the gap between prokaryotes and eukaryotes. Nature, 521(7551), pp.173179.

Stein, J.L., Marsh, T.L., Wu, K.Y., Shizuya, H. and DeLong, E.F., 1996. Characterization of uncultivated prokaryotes: isolation and analysis of a 40-kilobase-pair genome fragment from a planktonic marine archaeon. Journal of Bacteriology, 178(3), pp.591-9.

Stone, N.E., Fan, J.B., Willour, V., Pennacchio, L. a, Warrington, J. a, Hu, A., de la Chapelle, A., Lehesjoki, a E., Cox, D.R. and Myers, R.M., 1996. Construction of a 750-kb bacterial clone contig and restriction map in the region of human chromosome 21 containing the progressive myoclonus epilepsy gene. Genome 
Research, 6(3), pp.218-25.

Streif, S., Staudinger, W.F., Marwan, W. and Oesterhelt, D., 2008. Flagellar rotation in the archaeon Halobacterium salinarum depends on ATP. Journal of Molecular Biology, 384(1), pp.1-8.

Takai, K. and Nakamura, K., 2011. Archaeal diversity and community development in deep-sea hydrothermal vents. Current Opinion in Microbiology, 14(3), pp.282-291.

Takai, K., Sugai, A., Itoh, T. and Horikoshi, K., 2000. Palaeococcus ferrophilus gen. nov., sp. nov., a barophilic, hyperthermophilic archaeon from a deep-sea hydrothermal vent chimney. International Journal of Systematic and Evolutionary Microbiology, 50(2), pp.489-500.

Torsvik, V., Goksøyr, J. and Daae, F.L., 1990. High diversity in DNA of soil bacteria. Applied and Environmental Microbiology, 56(3), pp.782-7.

Tyson, G.W., Chapman, J., Hugenholtz, P., Allen, E.E., Ram, R.J., Richardson, P.M., Solovyev, V. V, Rubin, E.M., Rokhsar, D.S. and Banfield, J.F., 2004. Community structure and metabolism through reconstruction of microbial genomes from the environment. Nature, 428(6978), pp.37-43.

Tyson, G.W., Lo, I., Baker, B.J., Allen, E.E., Hugenholtz, P. and Banfield, J.F., 2005. Genome-directed isolation of the key nitrogen fixer Leptospirillum ferrodiazotrophum sp. nov. from an acidophilic microbial community. Applied and Environmental Microbiology, 71(10), pp.6319-24.

Verkhovskaya, M.L., Verkhovsky, M.I. and Wikström, M., 1995. A novel antiporter activity catalyzing sodium and potassium transport from right-side-out vesicles of $E$. 
coli. FEBS Letters, 363(1-2), pp.46-8.

Ward, D.M., Weller, R. and Bateson, M.M., 1990. 16S rRNA sequences reveal numerous uncultured microorganisms in a natural community. Nature, 345(6270), pp.63-5.

Waters, E., Hohn, M.J., Ahel, I., Graham, D.E., Adams, M.D., Barnstead, M., Beeson, K.Y., Bibbs, L., Bolanos, R., Keller, M., Kretz, K., Lin, X., Mathur, E., Ni, J., Podar, M., Richardson, T., Sutton, G.G., Simon, M., Soll, D., et al., 2003. The genome of Nanoarchaeum equitans: insights into early archaeal evolution and derived parasitism. Proceedings of the National Academy of Sciences of the United States of America, 100(22), pp.12984-12988.

Woese, C.R., Kandler, O. and Wheelis, M.L., 1990. Towards a natural system of organisms: proposal for the domains Archaea, Bacteria, and Eucarya. Proceedings of the National Academy of Sciences, 87(12), pp.4576-4579.

Wu, Y.-W., Tang, Y.-H., Tringe, S.G., Simmons, B. a and Singer, S.W., 2014. MaxBin: an automated binning method to recover individual genomes from metagenomes using an expectation-maximization algorithm. Microbiome, 2(1), p.26.

Wurch, L., Giannone, R.J., Belisle, B.S., Swift, C., Utturkar, S., Hettich, R.L., Reysenbach, A.-L. and Podar, M., 2016. Genomics-informed isolation and characterization of a symbiotic Nanoarchaeota system from a terrestrial geothermal environment. Nature Communications, 7, p.12115. 


\section{Appendix A: Supplemental information from the nanoarchaeotal and korarchaeotal genomic analysis}

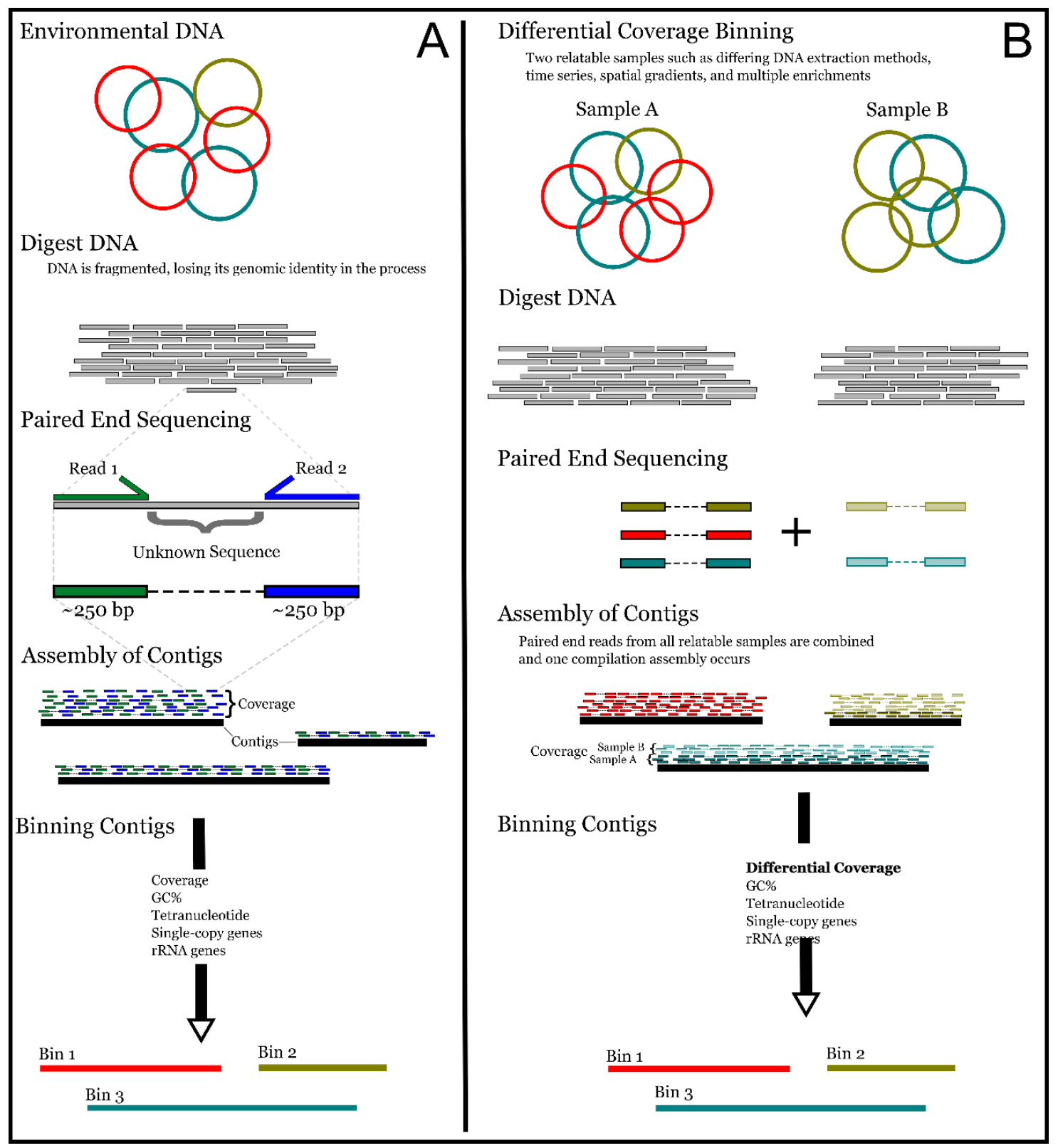

Figure 14. A. Overview of the shotgun sequencing process with an explanation of paired end sequencing. Paired reads are assembled into contigs through overlapping and stacking reads. Coverage is representative of genomic abundance. Various methods of binning help group the contigs in to bins. B. To use differential binning multiple, relatable samples are required. Paired end reads are combined for assembly. Reads are mapped back to the contigs individually to produce a multi-sample coverage profile used for binning. Differential coverage binning is still complemented with classical binning methods. 


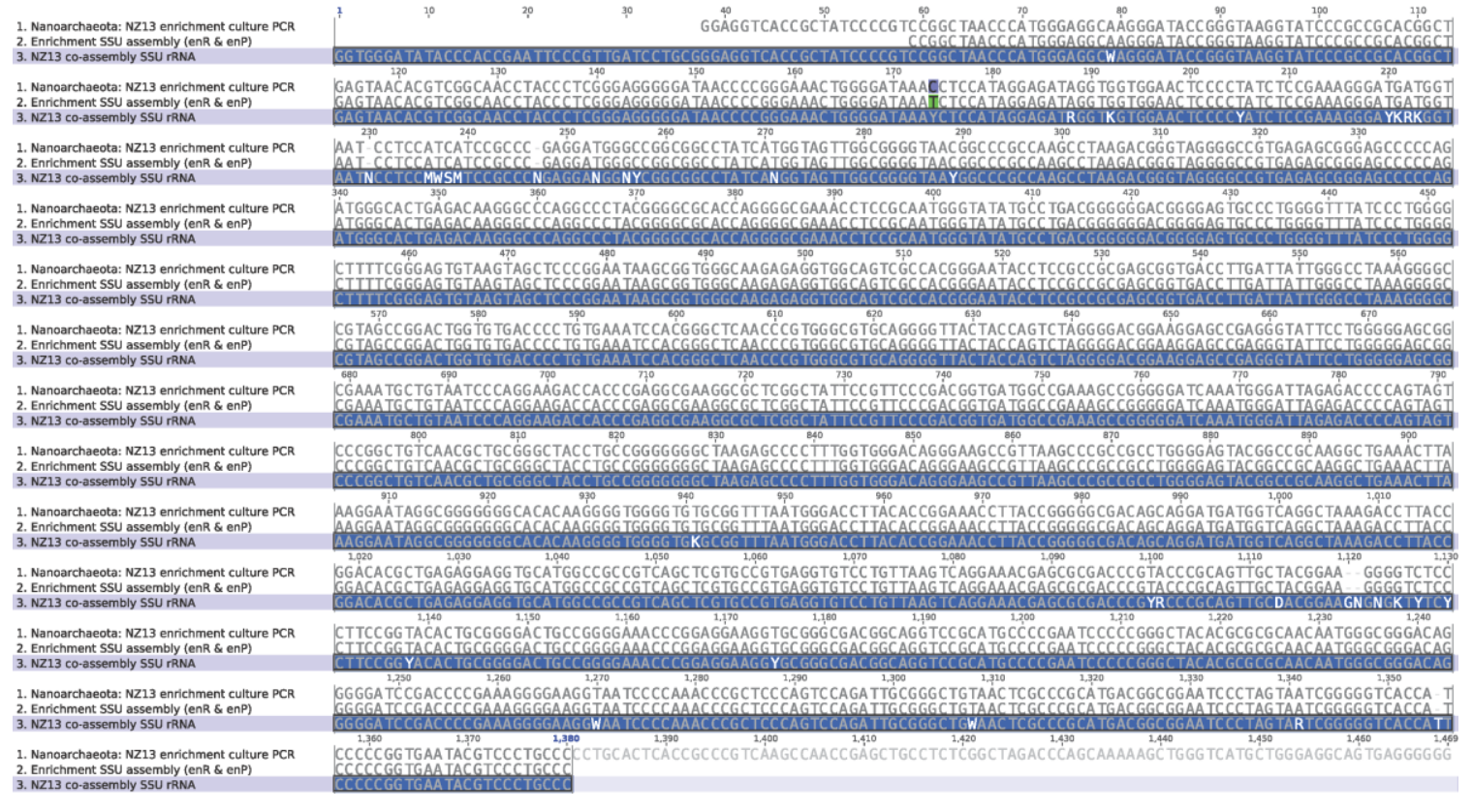

Figure 15. NZ13 nanoarchaeotal SSU rRNA sequence alignment. (1) The PCR sequence from an enrichment culture. (2) The de novo assembly of SSU rRNA from only NZ13en$\mathrm{R}$ and NZ13en-P reads. (3) The error prone sequence generated during assembly. 
Table 7. Distance identity map of nanoarchaeotal SSU rRNA sequences. NZ13en-R and NZ13 PCR are not $100 \%$ identical, due to a variable base pair change. See Figure 15.

\begin{tabular}{|c|c|c|c|c|c|}
\hline & N. equitans & Nst1 & N. acidilobi & $\begin{array}{l}\text { NZ13en-R } \\
\text { 16S rRNA }\end{array}$ & $\begin{array}{c}\text { NZ13 } \\
\text { enrichment }\end{array}$ \\
\hline N. equitans & & $81.05 \%$ & $81.12 \%$ & $84.95 \%$ & $83.48 \%$ \\
\hline Nst1 & $81.05 \%$ & & $97.47 \%$ & $89.02 \%$ & $87.63 \%$ \\
\hline N. acidilobi & $81.12 \%$ & $97.47 \%$ & & $89.17 \%$ & $87.77 \%$ \\
\hline $\begin{array}{l}\text { NZ13en-R } \\
\text { 16S rRNA }\end{array}$ & $84.95 \%$ & $89.02 \%$ & $89.17 \%$ & & $99.92 \%$ \\
\hline $\begin{array}{l}\text { NZ13 } \\
\text { enrichment }\end{array}$ & $83.48 \%$ & $87.63 \%$ & $87.77 \%$ & $99.92 \%$ & \\
\hline
\end{tabular}



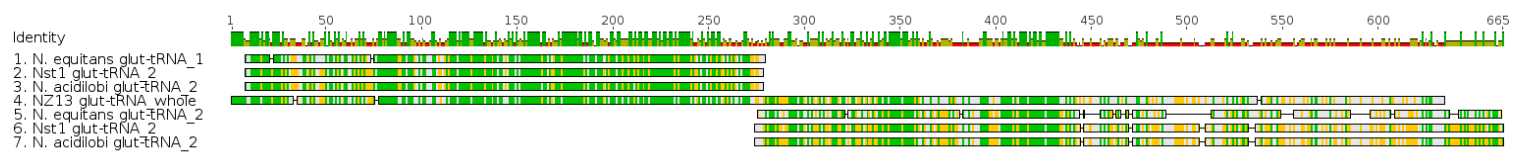

Figure 16. Protein alignment of Glu-tRNA ${ }^{\text {Gln }}$ amidotransferase. N. equitans $(1,5)$, Nst1 $(2,6)$ and $N$. acidilobi $(3,7)$ have a split gene, while NZ13 nanoarchaeote (4) is intact. 
Table 8. Relative abundance of amino acids present in the NZ13 nanoarchaeote. Open reading frames were predicted using Prodigal and individual amino acids were calculated.

\begin{tabular}{cccc}
\hline Relative abundance & Hits & Abbreviation & Amino Acids \\
\hline $4.0 \%$ & 6746 & $\mathrm{~A}$ & Alanine \\
$0.7 \%$ & 1194 & $\mathrm{C}$ & Cysteine \\
$5.1 \%$ & 8575 & $\mathrm{D}$ & Aspartic Acid \\
$8.4 \%$ & 14077 & $\mathrm{E}$ & Glutamic acid \\
$4.9 \%$ & 8278 & $\mathrm{~F}$ & Phenylalanine \\
$5.7 \%$ & 9505 & $\mathrm{G}$ & Glycine \\
$1.1 \%$ & 1910 & $\mathrm{H}$ & Histidine \\
$11.2 \%$ & 18829 & $\mathrm{I}$ & Isoleucine \\
$9.6 \%$ & 16152 & $\mathrm{~K}$ & Lysine \\
$10.0 \%$ & 16690 & $\mathrm{~L}$ & Leucine \\
$1.8 \%$ & 3035 & $\mathrm{M}$ & Methionine \\
$5.0 \%$ & 8359 & $\mathrm{~N}$ & Asparagine \\
$3.2 \%$ & 5420 & $\mathrm{P}$ & Proline \\
$1.5 \%$ & 2560 & $\mathrm{Q}$ & Glutamine \\
$5.1 \%$ & 8525 & $\mathrm{R}$ & Arginine \\
$6.1 \%$ & 10190 & $\mathrm{~S}$ & Serine \\
$3.7 \%$ & 6217 & $\mathrm{~T}$ & Threonine \\
$6.3 \%$ & 10596 & $\mathrm{~V}$ & Valine \\
$0.9 \%$ & 1565 & $\mathrm{~W}$ & Tryptophan \\
$5.5 \%$ & 9247 & $\mathrm{Y}$ & Tyrosine \\
\hline & & & \\
\hline
\end{tabular}


Table 9. Nanoarchaeotal genomic comparison table. Contig numbers are listed in the column below each respective Nanoarchaeota. All hits had an e-value greater than $10^{-5}$. Genes not detected in the genomes are denoted by '-'.

\begin{tabular}{|c|c|c|c|c|c|c|}
\hline Protein & $\begin{array}{l}\text { Gene } \\
\text { Name }\end{array}$ & EC & $\begin{array}{l}\text { Protein } \\
\text { Domain }\end{array}$ & $\begin{array}{c}\text { NZ13 } \\
\text { Nano- } \\
\text { archaeota } \\
\end{array}$ & $\begin{array}{c}\text { Nanopusillus } \\
\text { acidilobi }^{*}\end{array}$ & $\begin{array}{c}\text { Nanoarchaeum } \\
\text { equitans }\end{array}$ \\
\hline \multicolumn{7}{|l|}{ Glycogen } \\
\hline Glycogen synthase & & 2.4.1.11 & PF05693 & 0_16 & 75 & - \\
\hline Nucleotidyl transferase & & & PF00483 & $17 \_3$ & 321 & - \\
\hline $\begin{array}{l}\text { Glycogen debranching } \\
\text { enzyme }\end{array}$ & & 3.2.1.18 & PF03065 & 0_13 & 72 & - \\
\hline Glucan phosphorylase & & 2.4.1.1 & PF00343 & 4_12 & 585 & - \\
\hline $\begin{array}{l}\text { Phosphoglucose } \\
\text { mutase }\end{array}$ & pgm & 5.4.2.2 & PF02878 & $22 \_7$ & 326 & - \\
\hline \multicolumn{7}{|l|}{$\begin{array}{l}\text { Glycolysis - modified } \\
\text { Embden-Meyerhoff } \\
\text { pathway }\end{array}$} \\
\hline Glucokinase & glk & 2.7.1.2 & PF02685 & - & - & - \\
\hline $\begin{array}{l}\text { Glucose-6-Phosphate } \\
\text { isomerase }\end{array}$ & pgi & 5.3 .1 .9 & PF06560 & 0_96 & 483 & - \\
\hline 6-phosphofructokinase & pfk & 2.7.1.11 & PF00365 & - & - & - \\
\hline $\begin{array}{l}\text { Fructose bisphosphate } \\
\text { aldolase class I }\end{array}$ & pfk & 4.1.2.13 & PF00274 & - & - & - \\
\hline $\begin{array}{l}\text { Fructose bisphosphate } \\
\text { aldolase class II }\end{array}$ & pfk & 4.1.2.13 & PF01116 & - & - & - \\
\hline $\begin{array}{l}\text { Triosephosphate } \\
\text { isomerase } \\
\text { Glyceraldehyde-3- }\end{array}$ & tim & 5.3.1.1 & PF00121 & 112_1 & 469 & - \\
\hline $\begin{array}{l}\text { phosphate } \\
\text { dehydrogenase }\end{array}$ & gapn & 1.2 .1 .9 & PF00171 & - & 456 & - \\
\hline $\begin{array}{l}\text { Glyceraldehyde-3- } \\
\text { phosphate: ferredoxin } \\
\text { oxidoreductase }\end{array}$ & gapor & 1.2.7.6 & PF02730 & $\begin{array}{c}16 \_4 \\
26 \_10 \\
18 \_1\end{array}$ & $\begin{array}{l}415 \\
532 \\
145\end{array}$ & - \\
\hline $\begin{array}{l}\text { Phosphoglycerate } \\
\text { mutase }\end{array}$ & pam & 5.4 .2 .1 & PF10143 & 1_9 & 648 & - \\
\hline Enolase & eno & 4.2.1.11 & PF00113 & $0 \_102$ & 355 & - \\
\hline $\begin{array}{l}\text { Phosphoenolpyruvate } \\
\text { synthase }\end{array}$ & peps & 2.7.9.2 & PF01326 & $28 \_3$ & 513 & - \\
\hline Pyruvate kinase & $\mathrm{pk}$ & 2.7.1.40 & PF00224 & - & 327 & - \\
\hline $\begin{array}{l}\text { Pyruvate: phosphate } \\
\text { dikinase }\end{array}$ & ppdk & 2.7.9.1 & TIGR01828 & - & - & - \\
\hline $\begin{array}{l}\text { Pyruvate-ferredoxin } \\
\text { oxidoreductase }\end{array}$ & por & 1.2.7.1 & PF01855 & 18_7 18_6 & $\begin{array}{l}312 \\
340\end{array}$ & - \\
\hline $\begin{array}{l}\text { Acetyl_CoA synthetase } \\
\text { (NDP forming) }\end{array}$ & acs & 6.2 .1 .13 & TIGR02717 & $\begin{array}{l}19 \_11 \\
19 \_12\end{array}$ & $\begin{array}{l}503 \\
504\end{array}$ & - \\
\hline
\end{tabular}

\section{Gluconeogenesis}

Fructose-1,6-

bisphosphate class

fbpase 3.1.3.11

PF01950

10_4

58 
Glyceraldehyde 3-

phosphate

dehydrogenase

Phosphoglycerate

kinase

\section{Energy Metabolism}

ATPase

Inorganic

pyrophosphatase

Cytochrome bd oxidase

subunit I

\section{Transporter}

$\mathrm{ABC}$ transporter

$\mathrm{ABC}$ transporter -

ATPase

ABC transporter ATP-

binding

$\mathrm{ABC}$ transporter

$\mathrm{ABC}$ transporter

ABC-2 type transporter

$\mathrm{K}+$ transporter

$\mathrm{K}+$ transporter

Archaellum

Archaeal flagellin

Archaeal flagellin

Archaeal flagellin

Archaeal flagellin

Archaeal flagellar

protein $\mathrm{F}$

Archaeal flagellar

protein $\mathrm{D} / \mathrm{E}$

Flagellar accessory protein FlaH

Archaeal flagellar protein FlaI

Archaeal flagella

assembly protein $\mathrm{J}$

\section{CRISPER}

CRISPR-associated protein Cas1 - subtype

IB

CRISPR-associated protein Cas2

CRISPR-associated protein Cas3

CRISPR-associated protein Cas4 $\begin{array}{ccc} & & \text { PF02800 } \\ \text { gapdh } & 1.2 .1 .12 & \\ \text { pgk } & \text { 2.7.2.3 } & \text { PF00162 }\end{array}$

3.6.3.6 PF00122 21_8 112_2 470, 244

3.6.1.1 PF00719 15_12 413

PF01654 116

$\begin{array}{llll}\text { 1.10.3.- } & \text { PF01654 } & & \end{array}$

$\begin{array}{ccccc} & & & & \\ & \text { PF00005 } & 2 \_39 & 5 & 79 \\ & \text { PF00005 } & 33 \_3 & 659 & 312 \\ & \text { PF00005 } & 7 \_15 & 487 & 440 \\ & \text { PF00005 } & 19 \_6 & 280 & - \\ & \text { BLAST } & 107 \_1 & 270 & - \\ \text { trkA } & \text { PF01061 } & 33 \_4 & 486 & - \\ \operatorname{trkH} & \text { PF02254 } & 54 \_2 & - & - \\ & \text { PF02386 } & 54 \_3 & - & -\end{array}$

flab

flab

PF01917 3_14

PF01917 3_16

381

PF01917 3_19 384

flab

PF01917

4_9

655

BLAST

4_10

PF04659

22_2

183

BLAST

$22 \_4$

181

flah

BLAST

$22 \_5$

180

PF00482

flaj

$38 \_6$

445

PF01867

cas1

104_1

19

PF09827

cas2

TIGR01587

18

cas3

PF01930

cas4

21_1

24 


\begin{tabular}{|c|c|c|c|c|c|}
\hline $\begin{array}{l}\text { CRISPR-associated } \\
\text { protein Cas5 }\end{array}$ & cas5 & PF09704 & - & - & 22 \\
\hline $\begin{array}{l}\text { CRISPR-associated } \\
\text { protein Cas6 }\end{array}$ & cas6 & PF01881 & 21_2 & - & 1 \\
\hline $\begin{array}{l}\text { CRISPR-associated } \\
\text { protein Cas7 }\end{array}$ & cas7 & PF05107 & - & - & 21 \\
\hline \multicolumn{6}{|l|}{ Fe-S assembly } \\
\hline SufS & sufs & TIGR01979 & 3_12 & 495 & - \\
\hline SufA & sufa & TIGR01997 & - & - & - \\
\hline SufB & sufb & TIGR01980 & 7_16 & 385 & 138 \\
\hline SufC & sufc & BLAST & 7_15 & 270 & 79 \\
\hline SufD & sufd & PF01458 & 7_17 & 271 & - \\
\hline \multicolumn{6}{|l|}{$\begin{array}{l}\text { Sec protein } \\
\text { translocase }\end{array}$} \\
\hline $\operatorname{Sec} \mathrm{Y}$ & $\sec \mathrm{Y}$ & PF00344 & $4 \_5$ & 575 & 176 \\
\hline Sec61 & $\sec 61$ & PF03911 & $1 \_31$ & - & - \\
\hline $\mathrm{SecF}$ & $\operatorname{secF}$ & PF02355 & $26 \_3$ & 295 & 458 \\
\hline SecD & $\sec \mathrm{D}$ & PF02355 & 0_- 98 & - & 459 \\
\hline \multicolumn{6}{|l|}{ Polyamine } \\
\hline $\begin{array}{l}\text { Pyruvoyl-dependent } \\
\text { arginine decarboxylase }\end{array}$ & & PF02675 & 0_22 & 115 & - \\
\hline Agmatinase & & TIGR01230 & $0 \_33$ & 90 & 232 \\
\hline
\end{tabular}


1. NZ13_Korarchaeota_165 2. NZ13en-P-Korarchaeota_165

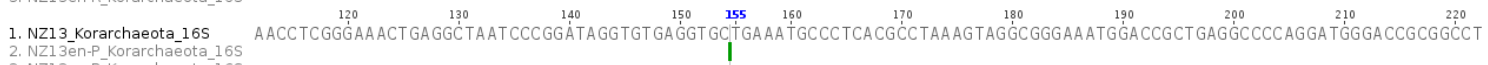
2. NZ13en-P.Korarchaeota_16S

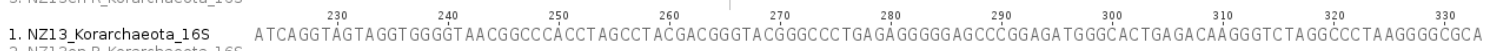
3. NZ13en-R Korarchaeota 165 .

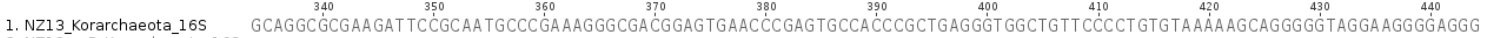
2. NZ13en-P. Korarchaeota_l6s

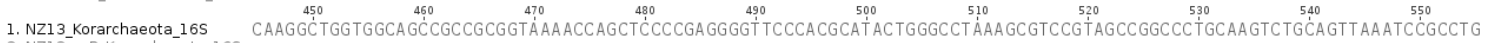

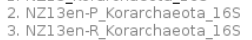

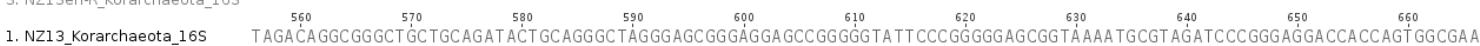

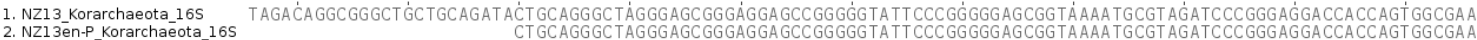
2. NZ13en-P.Korarchaeota_16S

1. NZ13_Korarchaeota_16S

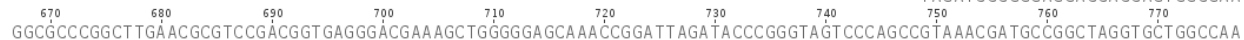
2. NZZ13en-10 1. NZ13 Korarcheeota_165 GG广

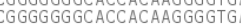
3. NZ13en-R-Korarcheota_-16S GGTTTCGGCCT TGGCCAGTGT CGAAGCGAAGGCATTAAGCCGGCCGCCTGAGGAGT ACGGCCGCAAGGCT GAAACTTGAAGGAAT TGACGGGGGGGCACCACAAGGGTGA

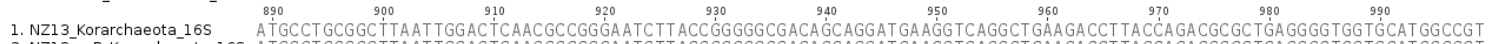

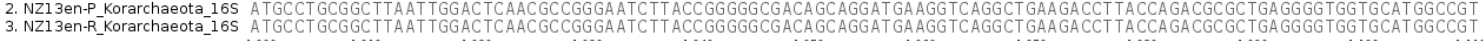

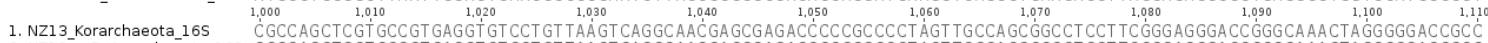
3. NZ13en-R-korarcheoota-165 CGCCAGCTCGT GCCGT GAGGTGTCCTGT TAAGTCAGGCAACGAGCGAGACCCCCGCCCCTAGTTGCCAGCGGCCTCCTTCGGGAGGGACCGGGCAAACTAGGGGGACCGCC

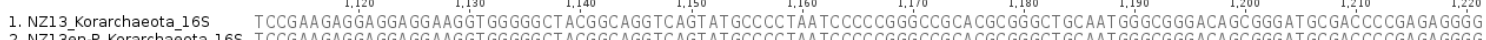

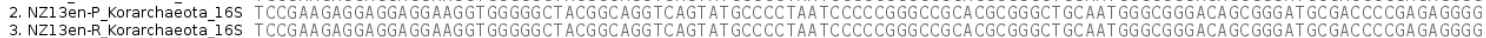

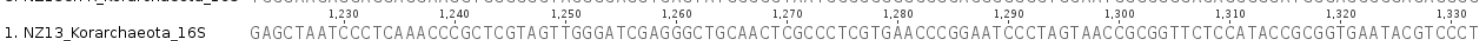

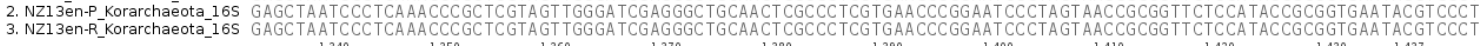

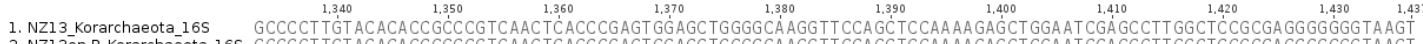
3. NZ13en-R.Korarchaeota_-16S GCCCCTTGTACACACCGCCCGTCAACTCACCCGAGTGGAGCTGGGGCAAGGTTCCAGCTCCAAAAGAGCTGGAATCGAGCCTTGGCTCCGCGAGGGGGGGTAAGT

Figure 17. NZ13 korarchaeotal consensus sequence alignment of SSU rRNA from (1) NZ13, (2) NZ13en-R and (3) NZ13en-P. 


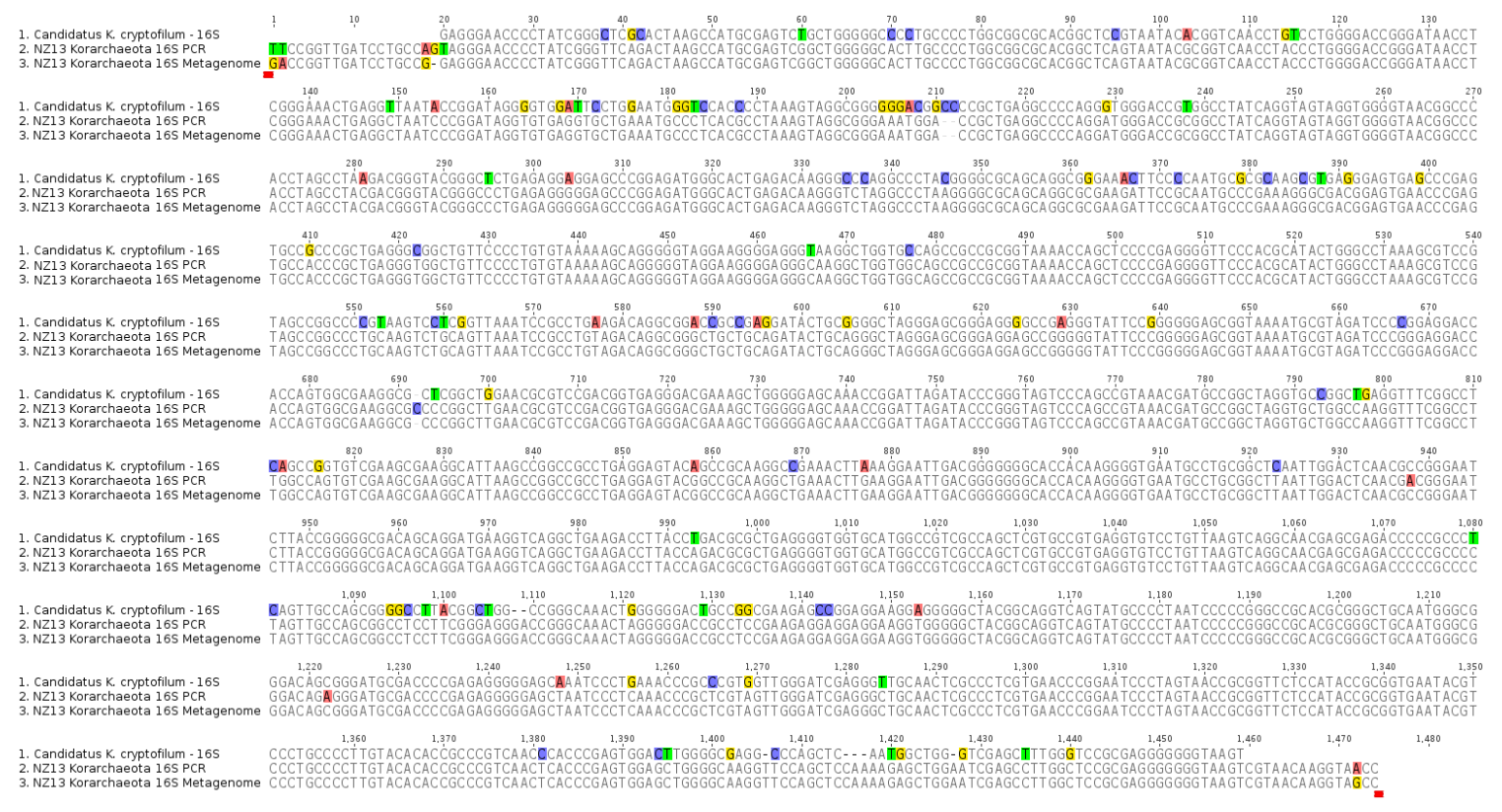

Figure 18. Sequence alignment of SSU rRNA of $\mathrm{Ca}$. K. cryptofilum, NZ13 Korarchaeota PCR sequence and NZ13 korarchaeotal metagenome consensus sequence. 
Table 10. Distance identity map of korarchaeotal SSU rRNA sequences. NZ13 Korarchaeota PCR and NZ13 korarchaeotal metagenome are not 100\% identical, due to many base pair changes. See Figure 17.

\begin{tabular}{l|c|c|c|} 
& $\begin{array}{c}\text { Candidatus } \\
\text { cryptofilum }\end{array}$ & $\begin{array}{c}\text { NZ13 } \\
\text { Korarchaeota } \\
\text { enrichment }\end{array}$ & $\begin{array}{c}\text { NZ13 } \\
\text { Korarchaeota } \\
\text { metagenome }\end{array}$ \\
$\begin{array}{l}\text { Candidatus K. } \\
\text { cryptofilum }\end{array}$ & $91.39 \%$ & $91.39 \%$ & $91.66 \%$ \\
$\begin{array}{l}\text { NZ13 } \\
\text { Korarchaeota } \\
\text { enrichment }\end{array}$ & $91.66 \%$ & & \\
\hline $\begin{array}{l}\text { NZ13 } \\
\text { Korarchaeota } \\
\text { metagenome }\end{array}$ & $99.39 \%$ & \\
\hline
\end{tabular}


Table 11. Korarchaeotal genomic comparison table. Contig numbers are listed in the column below each respective korarchaeote. All hits had an evalue greater than $10^{-5}$. Genes not present in the genomes are denoted by '-'.

\begin{tabular}{|c|c|c|c|c|c|c|}
\hline Protein & $\begin{array}{l}\text { Gene } \\
\text { Name } \\
\end{array}$ & EC & $\begin{array}{l}\text { Protein } \\
\text { Domain } \\
\end{array}$ & $\begin{array}{l}\text { NZ13 Kor- } \\
\text { archaeota }\end{array}$ & $\begin{array}{c}\text { Candidatus } \\
\text { Korarchaeum } \\
\text { cryptofilum* }\end{array}$ & $\begin{array}{c}\text { Little Hot } \\
\text { Creek }\end{array}$ \\
\hline \multicolumn{7}{|l|}{ Glycogen } \\
\hline $\begin{array}{l}\text { Glycogen synthase } \\
\text { Glycogen debranching } \\
\text { enzyme }\end{array}$ & & 2.4.1.11 & PF05693 & - & - & - \\
\hline Glucan phosphorylase & & 2.4.1.1 & PF00343 & - & - & - \\
\hline $\begin{array}{l}\text { Phosphoglucose mutase } \\
\text { Glycolysis - modified } \\
\text { EM pathway }\end{array}$ & Pgm & 5.4 .2 .2 & PF02878 & 3_22 & 1562 & 14949_1 \\
\hline $\begin{array}{l}\text { Glucokinase } \\
\text { Glucose-6-Phosphate } \\
\text { isomerase }\end{array}$ & Glk & 2.7.1.2 & PF02685 & 9_16 & 1071 & $1123 \_8$ \\
\hline 6-phosphofructokinase & Pfk & 2.7.1.56 & PF00365 & - & - & - \\
\hline $\begin{array}{l}\text { ATP-dependent } \\
\text { phosphofructokinase } \\
\text { Fructose bisphosphate }\end{array}$ & PfkB & 2.7.1.11 & PF00294 & 0_97, 63_8 & 1260,376 & $\begin{array}{l}825 \_18 \\
5083 \_4\end{array}$ \\
\hline $\begin{array}{l}\text { aldolase class I } \\
\text { Fructose bisphosphate }\end{array}$ & $\mathrm{Fba}$ & 4.1.2.13 & PF00274 & 036 & 1320 & 37251 \\
\hline $\begin{array}{l}\text { aldolase class II } \\
\text { Triosephosphate } \\
\text { isomerase }\end{array}$ & Tim & 4.1.2.13 & PF00121 & 1_13 & 1532 & $\begin{array}{l}3725 \_1 \\
4478 \_5\end{array}$ \\
\hline $\begin{array}{l}\text { Glyceraldehyde-3- } \\
\text { phosphate dehydrogenase } \\
\text { Glyceraldehyde-3- } \\
\text { phosphate: ferredoxin } \\
\text { oxidoreductase }\end{array}$ & Gapn & 1.2 .1 .9 & PF00171 & $4 \_42,38 \_1$ & 1132 & $\begin{array}{l}\text { 6772_1, } \\
29715 \_2 \\
1955 \_3, \\
15344 \_2\end{array}$ \\
\hline Phosphoglycerate mutase & Pgam & 5.4.2.1 & PF10143 & 0_89 & 1268 & 825_10 \\
\hline Enolase & Eno & 4.2.1.11 & PF00113 & $5 \_14$ & 1672 & $2240 \_2$ \\
\hline $\begin{array}{l}\text { Phosphoenolpyruvate } \\
\text { synthase }\end{array}$ & Peps & 2.7.9.2 & PF01326 & $59 \_10$ & 61 & $\begin{array}{l}\text { 6462_1 } \\
1636 \_3\end{array}$ \\
\hline $\begin{array}{l}\text { Pyruvate kinase } \\
\text { Pyruvate: phosphate } \\
\text { dikinase }\end{array}$ & Ppdk & 2.7.1.40 & $\begin{array}{l}\text { PF00224 } \\
\text { TIGR0182 } \\
8\end{array}$ & $2 \_1,92 \_5$ & 155 & 2671_4 \\
\hline $\begin{array}{l}\text { Pyruvate-ferredoxin } \\
\text { oxidoreductase } \\
\text { Acetyl_CoA synthetase } \\
\text { (NDP forming) }\end{array}$ & Por & 6.2 .1 .13 & $\begin{array}{c}\text { PF01855 } \\
\text { TIGR0271 } \\
7 \\
\end{array}$ & $\begin{array}{l}47-3,0 \_9, \\
0 \_41,0 \_76\end{array}$ & $\begin{array}{c}1280,716,1315 \\
1346,75 \\
31,76,204,293, \\
1187 \\
\end{array}$ & $\begin{array}{c}\text { 5199_6, } \\
737 \_3\end{array}$ \\
\hline \multicolumn{7}{|l|}{ Gluconeogenesis } \\
\hline $\begin{array}{l}\text { Fructose-1,6-bisphosphate } \\
\text { class V }\end{array}$ & Fbpase & 3.1.3.11 & PF01950 & 1_12 & 1531 & $\begin{array}{l}\text { 4478_6, } \\
7484 \_1\end{array}$ \\
\hline Glyceraldehyde 3- & Gapdh & 1.2.1.12 & PF02800 & $54 \_2$ & 238 & 3737_2 \\
\hline
\end{tabular}


phosphate dehydrogenase

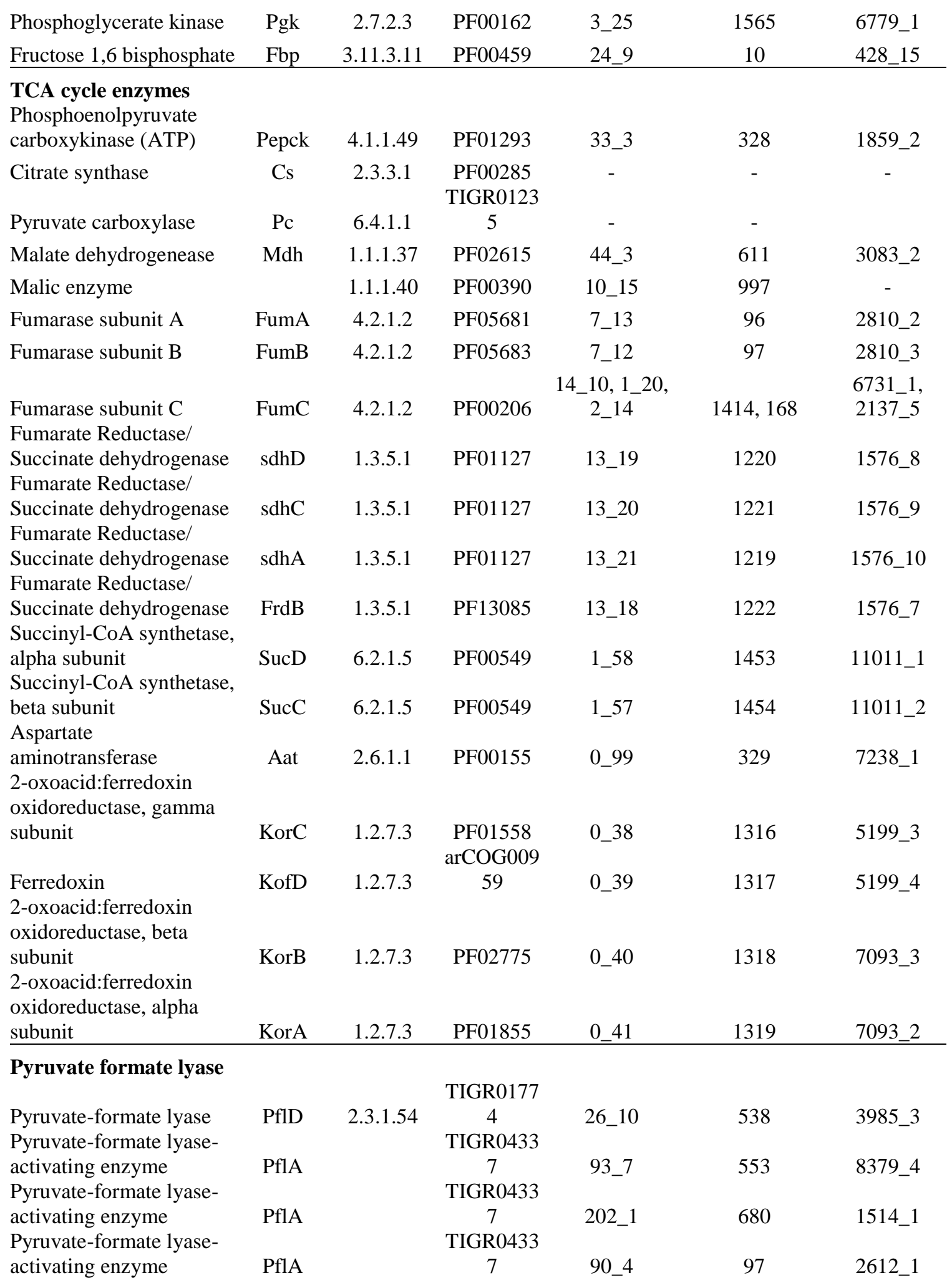


Pyruvate-formate lyaseactivating enzyme

Pyruvate-formate lyase-

activating enzyme

Pyruvate-formate lyase-

activating enzyme

TIGR0433

Pentose Pathway -

RuMP pathway

3-hexulose-6-phosphate

isomerase

PflA

7

PflA

TIGR0249

TIGR0249

91_5 969

6514_5

26_11 284

3985_4

PflA

157_2

539

4645_2

3-hexulose-6-phosphate

synthase

Ribose 5-phosphate

isomerase

ribose-phosphate

pyrophosphokinase

\section{Urea Cycle}

Carbamate kinase

2.7.2.2 PF00696

0_53

1303

1846_2

Ornithine

carbamoyltransferase

Argininosuccinate

synthae

$\begin{array}{lcc}\text { HxlB } & 5.3 .1 .27 & 7\end{array}$

5_12

1674

2240_4

Hps $\quad$ 4.1.2.43 PF00215

8_8

1361

4319_3

RpiA

5.3.1.6 PF06026

5_1

2

428_7

PrsA

2.7.6.1

PF00156

0_95

1262

825_16

Argininosuccinate lyase

Arginine deiminase

\begin{tabular}{|c|c|c|c|c|c|}
\hline $\operatorname{ArgF}$ & 2.1.3.3 & $\begin{array}{c}\text { TIGR0065 } \\
8 \\
\text { arCOG001 }\end{array}$ & $\begin{array}{c}0 \_52,67 \_3, \\
8 \_34\end{array}$ & $1387,522,1304$ & $\begin{array}{l}2916 \_4, \\
4393 \_1\end{array}$ \\
\hline $\operatorname{ArgG}$ & 6.3.1.5 & $\begin{array}{c}12 \\
\text { TIGR0083 }\end{array}$ & 2_15 & 169 & 6731_2 \\
\hline $\mathrm{ArgH}$ & 4.3.2.1 & $\begin{array}{c}8 \\
\operatorname{arCOG} 031\end{array}$ & $2 \_14$ & 168 & 6731_1 \\
\hline $\operatorname{ArcA}$ & 3.5.3.6 & 08 & 67_5 & 524 & 2916_2 \\
\hline
\end{tabular}

\section{Energy Metabolism}

\section{ATPase}

Archaeal/vacuolar-type

H+-ATPase subunit A

Archaeal/vacuolar-type

H+-ATPase subunit A

Archaeal/vacuolar-type

H+-ATPase subunit B

Archaeal/vacuolar-type

H+-ATPase subunit C

Archaeal/vacuolar-type

H+-ATPase subunit D

Archaeal/vacuolar-type

H+-ATPase subunit E

Archaeal/vacuolar-type

$\mathrm{H}+$-ATPase subunit $\mathrm{H}$

Archaeal/vacuolar-type

H+-ATPase subunit I

Archaeal/vacuolar-type

H+-ATPase subunit I

Archaeal/vacuolar-type

H+-ATPase subunit I

Archaeal/vacuolar-type

H+-ATPase subunit I

$\begin{array}{cccccc}\text { NtpA } & 3.6 .3 .14 & \text { PF02874 } & 0 \_1 & - & - \\ \text { NtpA } & 3.6 .3 .14 & \text { PF02874 } & 8 \_1 & 1354 & 3143 \_4 \\ \text { NtpB } & 3.6 .3 .14 & \begin{array}{c}\text { PF02874 } \\ \text { arCOG024 }\end{array} & 8 \_2 & 1355 & 3143 \_5 \\ \text { NtpC } & 3.6 .3 .14 & 59 & 8 \_4 & 1357 & 3143 \_7 \\ \text { NtpD } & 3.6 .3 .14 & \text { PF01813 } & 8 \_3 & 1356 & 3143 \_6 \\ \text { NtpE } & 3.6 .3 .14 & \begin{array}{c}\text { arCOG008 } \\ \text { arCOG033 }\end{array} & 0 \_2 & 1353 & 3143 \_3 \\ \text { NtpF } & 3.6 .3 .14 & 63 & 0 \_3 & 1352 & 3143 \text { _2 } \\ \text { NtpI } & 3.6 .3 .14 & \text { PF01496 } & 8 \_6 & 1359 & 4319 \_1 \\ \text { NtpI } & 3.6 .3 .14 & \text { PF01496 } & 95 \_3 & 1122 & 2123 \_2 \\ \text { NtpI } & 3.6 .3 .14 & \text { PF01496 } & 72 \_6 & - & - \\ \text { NtpI } & 3.6 .3 .14 & \text { PF01496 } & 50 \_7 & - & -\end{array}$


Archaeal/vacuolar-type

$\mathrm{Na}+/ \mathrm{H}+-\mathrm{ATPase}$, subunit

KADH dehydrogenase

NADH dehydrogenase

subunit A

NADH dehydrogenase

subunit B

NADH dehydrogenase

subunit $\mathrm{C}$

NADH dehydrogenase

subunit D

NADH dehydrogenase

subunit $\mathrm{H}$

NADH dehydrogenase

subunit I

NADH dehydrogenase

subunit $\mathbf{J}$

NADH dehydrogenase

subunit 4L (K,kappa)

NADH:ubiquinone

oxidoreductase subunit 4

(chain M)

AtpK $\quad 3.6 .3 .14 \quad \begin{array}{ll}\operatorname{arCOG024} \\ 55\end{array}$

8

1358

NADH dehydrogenase

subunit L

NADH dehydrogenase

subunit L

NADH dehydrogenase

subunit N

$\begin{array}{ccc} & \text { arCOG015 } \\ \text { NuoA } & 1.6 .5 .3 & 57\end{array}$

6_27

141

1485_5

NuoB $\quad$ 1.6.5.3 PF01058

6_28

142

1485_4

NuoC

1.6.5.3 PF00329

6_29

143

1485_3

NuoD $\quad$ 1.6.5.3 PF00346

6_30

144

1485_2

NuoH

1.6.5.3 PF00146

6_31

145

16741_2

NuoI $\quad 1.6 .5 .3 \quad 43$

6_32

146

16741_2

$\begin{array}{lc}\text { NuoJ } & \text { arCOG046 } \\ \text { 1.6.5.3 } & 54\end{array}$

6_33

147

16741_3

NuoK 1.6.5.3 73

6_34

148

1478_10

NuoM $\quad$ 1.6.5.3 $\quad$ PF00361

$6 \_35$

149

1478_7

NuoL

1.6.5.3 PF00361

6_36

150

$1478 \_8$

NuoL 1.6.5.3 PF00361 92_1

151

NuoN $\quad$ 1.6.5.3 PF00361

92_2

225

1478_9

\section{Heterodisulfide}

Reductase

Heterodisulfide reductase,

subunit B

Heterodisulfide reductase,

subunit C

Heterodisulfide reductase,

subunit A

Heterodisulfide reductase,

subunit A

Heterodisulfide reductase,

subunit A

Heterodisulfide reductase, subunit E, CoB-Com

F420-Reducing

Hydrogenase

Coenzyme F420-reducing

hydrogenase, alpha

subunit

FrhA

PF00374

33_6

332

4115_1

Coenzyme F420-reducing hydrogenase, gamma

subunit

FrhG

PF01058

4_14

333

4813_4

Coenzyme F420-reducing

hydrogenase, gamma

FrhG

PF01058 33_7

1166 
subunit

Coenzyme F420-reducing hydrogenase, delta subunit FrhD $\operatorname{arCOG024}$

Coenzyme F420-reducing hydrogenase, delta

subunit

Fri

75

4_15

1165

Carbon-monoxide

Dehydrogenase

carbon-monoxide

dehydrogenase, catalytic

subunit

FrhD

arCOG024

$\mathrm{CO}$ dehydrogenase

maturation factor

CooC

PF03063

49_1

801

9012_3

Formate Dehydrogenase

Protein involved in

formate dehydrogenase

formation

FdhE

arCOG056

formate dehydrogenase -

subunit B

FdhB

PF01656

49_2

802

9012_2

Menaquinone -

alternate Mevalonate

pathway

Acetyl-CoA C-

acetyltransferase

Hydroxymethylglutaryl-

CoA synthase

Hydroxymethylglutaryl-

CoA reductase

Mevalonate kinase

Phosphomevalonate

decarboxylase

Isopentenyl-diphosphate

Isopentenyl-diphosphate

isomerase

1,4-Dihydroxy-2-

naphtoate

prenyltransferase

Demethylmenaquinone

methyltransferase

4-hydroxybenzoate

polyprenyltransferase

PaaJ

PksG

2.3.3.10

HMG1

1.1 .1 .88

ERG12 2.7.1.36

Pmd

4.1.1.99

Ipk

2.7.4.26

Ipp

$$
\text { 5.3.3.2 }
$$

MenA

UbiE/Me

$n G$

2.5.1.74

2.1.1.163

UbiA
$\operatorname{arCOG012}$

BLAST
4_7

123_2
1173

1107_7

821

\section{Hydrogenase}

$\mathrm{Ni}, \mathrm{Fe}$-hydrogenase I large subunit

$\mathrm{Ni}, \mathrm{Fe}$-hydrogenase

maturation factor

$\mathrm{Ni}, \mathrm{Fe}$-hydrogenase

maturation factor

$4 \mathrm{Fe}-4 \mathrm{~S}$ ferredoxin iron-

HyaB

HyaD

HyaD

HybA
78

PF08545

PF00368

arCOG010

28

COG3407

arCOG008

60 arCOG006

13

$\operatorname{arCOG004}$

80

arCOG043

48

arCOG004
$13 \_13$

13_14

203_3

35_3

5_25

9_23

70_5, 68_2

$-$

3_4,65_5

507, 1544
675_9,

1227

1226

585

1115

3841_6

9728_1

14454_2

1123_1

1064

835

3560_2

\section{.}

$\begin{array}{cccc}\text { PF00374 } & \text { 4_13 } & 1167 & 1107 \_2 \\ \text { PF01750 } & \text { 4_12 } & 1168 & 1107 \_6 \\ \text { PF01750 } & \text { 33_5 } & 331 & \text { 4115_2 } \\ \text { TIGR0295 } & \text { 4_43 } & 1131 & 1955 \_2\end{array}$


sulfur binding domain

protein

Zn finger protein

HypA/HybF (possibly

regulating hydrogenase

expression)

Hydrogenase maturation

factor

Hydrogenase maturation

factor

Hydrogenase maturation

factor

Hydrogenase maturation

factor

Hydrogenase maturation

factor

Hydrogenase maturation

factor

\section{Cytochrome}

cytochrome bd-type

quinol oxidase subunit 1-

like protein

cytochrome bd ubiquinol

oxidase subunit I

Cytochrome b/b6 domain

protein

Rieske Fe-S protein

Bacterioferritin

(cytochrome b1)

Electron transfer

proteins

Electron transfer

flavoprotein alpha/beta-

subunit

Electron transfer

flavoprotein alpha/beta-

subunit

Electron transfer

flavoprotein alpha subunit FixB

Electron transfer

flavoprotein alpha subunit

geranylgeranyl reductase

geranylgeranyl reductase

monooxygenase FAD-

binding

Dehydrogenase

(flavoprotein)

Dehydrogenase

(flavoprotein)

FixC

FixC

FixC

FixC
1

\begin{tabular}{lcccc} 
HybF & PF01155 & 4_11 & 1169 & $1107 \_11$ \\
HypC & PF01455 & $4 \_9$ & 1171 & $1107 \_9$ \\
HypD & PF01924 & $4 \_20$ & 1160 & $9763 \_2$ \\
HypE & PF00586 & $4 \_8$ & 1172 & $1107 \_8$ \\
HypE & PF00586 & $32 \_5$ & 233 & 6319_1 \\
HypE & PF00586 & $12 \_24$ & 1185 & $1675 \_7$ \\
HypF & PF00708 & $4 \_19$ & 1161 & $1107 \_12$ \\
\hline
\end{tabular}

$\begin{array}{llll}\text { CydA } & \text { PF01654 5_37 } & 1649 & 5788 \_1\end{array}$

$\begin{array}{llll}\text { CydA } & \text { PF01654 5_36 } & 1650 & \text { 5788_2 }\end{array}$

$\begin{array}{llll}\text { QcrB } & \text { PF13631 13_24 7179_1 }\end{array}$

$\begin{array}{llll}\text { QcrA } & \text { PF00355 } & 13 \_23 & 1217\end{array}$

arCOG010

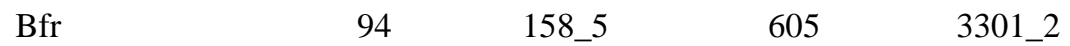

$\begin{array}{llll}\text { FixA } & \text { PF01012 9_1 } & 1085 & 9229 \_3\end{array}$

$\begin{array}{llll}\text { FixA } & \text { PF01012 32_6 } & 232 & \text { 8913_3 }\end{array}$

PF01012

PF00766 32_7 $1086 \quad$ 8913_2

PF01012

PF00766

119_1

231

9229_2

PF13450

$1 \_25$

212

2227_8

PF13450

$12 \_14$

59

6050_3

PF13450

$34 \_6$

1195

6127_5

PF13450

48_11

1419

10480_5

PF13450

59_12

1636_1

\section{Sec protein translocase}

SecY

$\sec \mathrm{Y}$

PF00344

$-$

1639

6621_5 


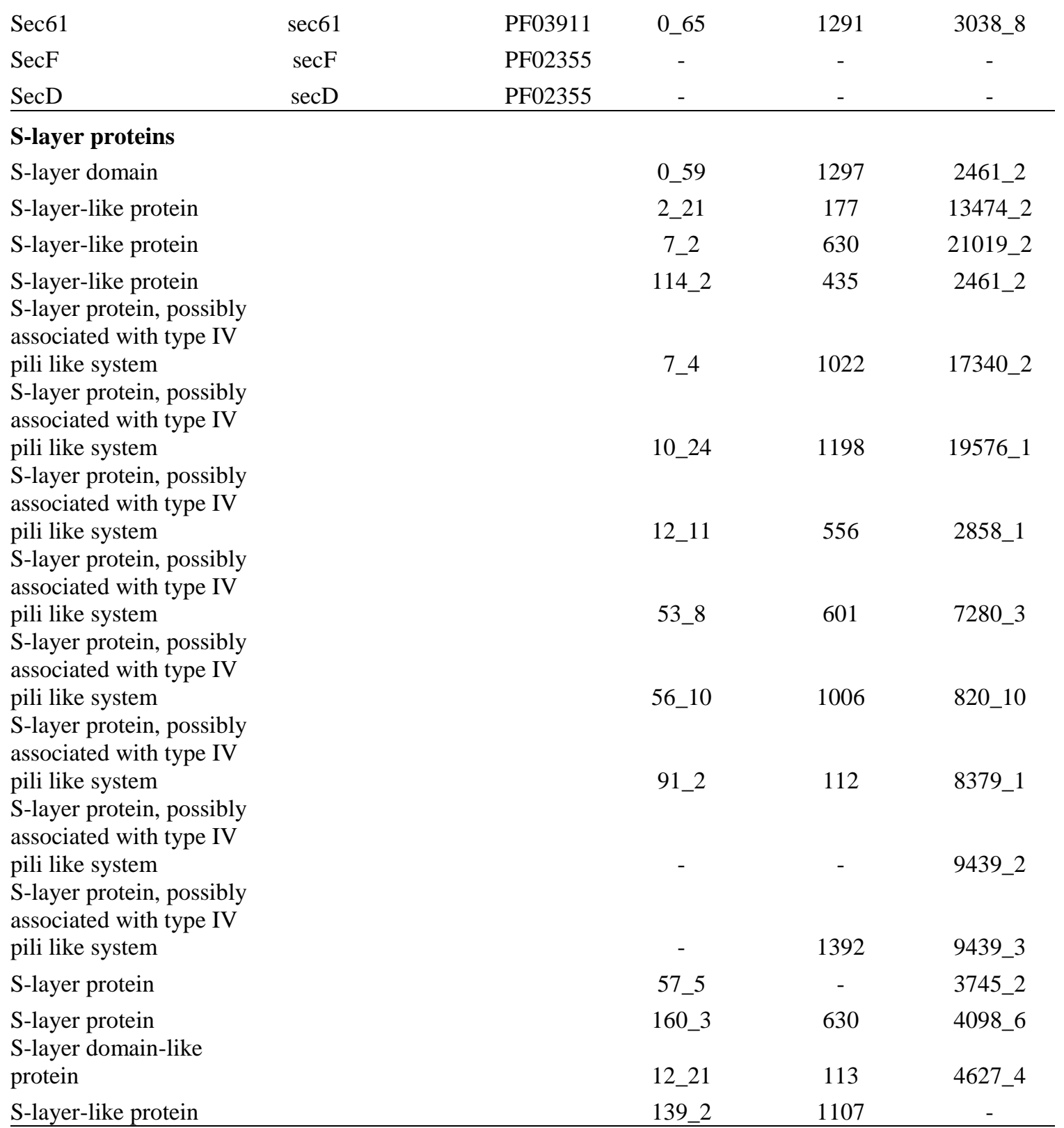

\section{CRISPER}

CRISPR-associated protein Cas1 protein Cas2

CRISPR-associated protein Cas 3

$\begin{array}{ccccc}\text { cas1 } & \text { PF01867 } & - & 439 & - \\ \text { cas2 } & \text { PF09827 } & - & 441 & \text { 27922_2 } \\ \text { cas3 } & \text { TIGR0158 } & - & 449 & 1843 \_8 \\ \text { cas4 } & 7 & - & 442 & \text { 6649_2 } \\ \text { cas5 } & \text { PF01930 } & 259 \_2 & 446 & \text { 14137_4 } \\ \text { cas6 } & \text { PF09704 } & - & 81 & \text { 2330_4 }\end{array}$

CRISPR-associated protein Cas4 protein Cas5

CRISPR-associated protein Cas6

d


CRISPR-associated protein Cas7

cas7

cmr5

protein $\mathrm{Cmr} 5$

CRISPR-associated protein $\mathrm{Cmr} 3$ $\mathrm{cmr} 3$
PF05107

PF09701

PF09700
461

459

\section{Transporter}

ABC-type

dipeptide/oligopeptide/nic kel transport system,

ATPase component

ABC-type oligopeptide

DppD

PF00005

0_47

1110

12421_2

transport system, ATPase

component

$\mathrm{AppF}$

PF00005

0_48

1111

1514_8

ABC-type

dipeptide/oligopeptide/nic kel transport system,

ATPase component

ABC-type oligopeptide

DppD

PF00005

0_58

1144

15269_2

transport system, ATPase

component

$\mathrm{AppF}$

PF00005

$1 \_47$

1145

1669_13

Energy-coupling factor

transporter ATP-binding

protein EcfA2

EcfA2

PF00005

1_48

1156

16996_1

ABC-type multidrug transport system, ATPase component

CcmA

PF00005

12_10

1190

1742_4

ABC-type multidrug transport system, ATPase component

$\mathrm{CcmA}$

PF00005

12_19

1199

1846_7

ABC-type antimicrobial peptide transport system, ATPase component ABC-type branched-chain SalX

PF00005

16_13

1298

1846_8 amino acid transport system, ATPase component

ABC-type branched-chain amino acid transport system, ATPase component

LivF

PF00005

17_22

1309

22872_3

Energy-coupling factor transporter ATP-binding protein EcfA2

EcfA2

PF00005

2_29

2426_2

ABC-type $\mathrm{Mn} / \mathrm{Zn}$

transport system, ATPase component

ZnuC

PF00005

204_1

186

2426_3

ABC-type multidrug transport system, ATPase component

CcmA

PF00005

204_2

425

2461_3

ABC-type

nitrate/sulfonate/bicarbon ate transport system,

TauB

PF00005 208_4

505

24788_2 
ATPase component

ABC-type oligopeptide

transport system, ATPase component

AppF

PF00005

25_14

527

3558_8

ABC-type

dipeptide/oligopeptide/nic kel transport system,

ATPase component

DppD

PF00005

27_15

528

3841_1

ABC-type multidrug

transport system, ATPase

component

CcmA

PF00005

27_16

563

3841_2

ABC-type multidrug

transport system, ATPase

component

CcmA

PF0000

342_1

671

4356_4

ABC-type multidrug

transport system, ATPase

component

CcmA

PF00005

342_2

726

4837_3

ABC-type

nitrate/sulfonate/bicarbon

ate transport system,

ATPase component

ABC-type multidrug

TauB

PF0000

35_8

780

6376_1

transport system, ATPase

component

CcmA

PF00005

35_9

832

6649_3

ABC-type branched-chain

amino acid transport

system, ATPase

component

LivG

PF00005

38_4

840

7280_4

ABC-type branched-chain amino acid transport

system, ATPase

component

LivF

PF00005

4_24

ABC-type branched-chain amino acid transport

system, ATPase

component

LivF

PF00005

42_8

85

7812_2

ABC-type branched-chain amino acid transport system, ATPase component

PF00005

60_9

86

7812_3

ABC-type branched-chain amino acid transport

system, ATPase

component

LivF

PF00005

61_3

866

9197_1

ABC-type branched-chain amino acid transport

system, ATPase

component

LivG

PF00005

7_23

867

9197_2

ABC-type sugar transport

system, ATPase

component

MalK

PF00005

7_24

918

9535_2

ABC-type phosphate

PstB

PF00005

75_8

962

9535_3 
component

ABC-type

dipeptide/oligopeptide/nic

kel transport system,

ATPase component

ABC-type oligopeptide

DppD

PF00005

80_4

976

19041_2

transport system, ATPase

component

$\mathrm{AppF}$

PF00005

$80 \_5$

977

2362_4

-type

uncharacterized transport

system, ATPase

component

PF00005

40_9

1079

2725_1

Energy-coupling factor

transporter ATP-binding

protein EcfA2

EcfA2

PF00005

47_5

1442

4519_1

ABC-type

uncharacterized transport

system, ATPase

component

PF00005

65_10

320

6096_1

Energy-coupling factor

transporter ATP-binding

protein EcfA2

EcfA2

PF00005

9_8

348

7238_3

Energy-coupling factor

transporter ATP-binding

protein EcfA2

EcfA2

PF00005

411

Energy-coupling factor

transporter ATP-binding

protein EcfA2

EcfA2

PF00005

698

copper--translocating P-

type ATPase/heavy metal-

(Cd/Co/Hg/Pb/Zn)

ZntA

PF00403

96_5

Amino acid transporter

PotE

PF00324

523

2916_3

ABC-type transport

system, periplasmic

component

$\operatorname{DdpA}$

PF00496

26_1

1141

16523_1

ABC-type transport

system, periplasmic

component

$\operatorname{DdpA}$

PF00496

531

2612_5

ABC-type transport

system, periplasmic

component

$\operatorname{DdpA}$

PF00496

973

ABC-type amino acid

transport/signal

transduction system

HisJ

PF00497

305

2142_5

ABC-type

dipeptide/oligopeptide/nic

kel transport system,

permease component

ABC-type

DppB

PF00528

16_10

1108

16523_2

dipeptide/oligopeptide/nic kel transport system, permease component

ABC-type

DppC

PF00528

17_15

1109

16996_3

DppB

PF00528

17_17

1142

2142_2 
dipeptide/oligopeptide/nic kel transport system, permease component ABC-type amino acid transport system, permease component

HisM

PF00528

204_4

302

2142_4

ABC-type amino acid transport system, permease component ABC-type

HisM

PF00528

35_10

304

24788_1

dipeptide/oligopeptide/nic kel transport system, permease component ABC-type

DppB

PF00528

$35 \_11$

530

2612_6

dipeptide/oligopeptide/nic kel transport system, permease component ABC-type anion transport DppB

PF00528

974

system, duplicated permease component ABC-type multidrug transport system, ATPase and permease component

MdlB

PF00664

26967_1

Cation transport ATPase MgtA

PF00690

Permease of the drug/metabolite transporter (DMT) superfamily

RhaT

PF00892

203_2

586

25176_2

drug/metabolite transporter (DMT)

superfamily

PF00892

$65 \_2$

110

4073_7

drug/metabolite transporter (DMT)

superfamily

RhaT

PF00892

-

509

14121_4

Permease of the drug/metabolite transporter (DMT) superfamily

RhaT

PF00892

17340_1

Permease of the drug/metabolite transporter (DMT) superfamily

RhaT

PF00892

20484_1

ABC-type Mn2+/Zn2+ transport system, permease component Kef-type K+ transport

PF00950

$2 \_28$

185

3558_7

system, membrane component

KefB

PF00999

20_5

546

6925_1

Kef-type K+ transport system, membrane component

KefB

PF00999 20_6 
ABC-type multidrug transport system, permease component ABC-type multidrug transport system, permease component ABC-type multidrug transport system, permease component ABC-type multidrug transport system, permease component ABC-type metal ion transport system, periplasmic component/surface adhesin

LraI

$$
\text { PF01061 }
$$

12_20

1189

15269_1

ABC-type transport system, periplasmic component/surface lipoprotein

$\mathrm{Mg} 2+$ and $\mathrm{Co} 2+$

transporter

Predicted $\mathrm{Co} / \mathrm{Zn} / \mathrm{Cd}$ cation transporter Predicted $\mathrm{Co} / \mathrm{Zn} / \mathrm{Cd}$ cation transporter

Permease, similar to cation transporter

Permease, similar to cation transporter

ABC-type $\mathrm{Co} 2+$ transport system, permease

component

CbiM

Med

PF01061

16_11

424

26249_2

PF01061 16_12

564

PF01061 25_13

Multiple antibiotic

transporter

MarC

CorA

PFo1456

83_5

321

27098_1

MMT1

PF01544

771

Predicted $\mathrm{Fe} 2+/ \mathrm{Mn} 2+$

MMT1

PF01545

161_4

557

12748_1

MgtE2

PF01545

91_1

19576_2

MgtE2

PF01769

21_4

1742_9

PF01769

4067_5

transporter, VIT1/CCC1

family

CCC1

PF01988

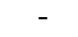

1675

2240_5

$\mathrm{Fe} 2+$ transport system protein $\mathrm{B}$

FeoB

PF07670

19_12

1617

17285_1

Predicted divalent heavy-

PF02535

27_2

223

1478_4

ABC-type transport

system, periplasmic

component/surface

lipoprotein

Med

PF02608

$9 \_11$

1076

1123_13

Uncharacterized ABC-

type transport system,

permease component

PF02653

0_49

1077

1846_5

ABC-type

uncharacterized transport system, permease

PF02653

0_50

1078

1846_6 
component

Branched-chain amino acid ABC-type transport system, permease component

ABC-type branched-chain amino acid transport system, permease component

uncharacterized transport system, permease

component

$27 \_14$

319

2426_4

Branched-chain amino acid ABC-type transport system, permease component

ABC-type branched-chain amino acid transport system, permease component acid ABC-type transport system, permease component

ched-chain amino acid ABC-type transport system, permease component

ABC-type branched-chain amino acid transport system, permease component acid ABC-type transport system, permease

Branched-chain amino acid ABC-type transport system, permease component

ABC-type antimicrobial peptide transport system, permease component 
family

$\mathrm{Na}+\mathrm{H}+$ antiporter $\mathrm{NhaD}$

or related arsenite

permease

ArsB

PF03600

0_32

$\mathrm{Na}+\mathrm{H}+$ antiporter $\mathrm{NhaD}$

or related arsenite

permease

ArsB

PF03600

20_11

Fe2+ transport system

protein $\mathrm{A}$

FeoA

PF04023

1616

2345_2

ABC-type multidrug

transport system,

permease component

PF04123

172_3

1093

1132_7

ABC-type multidrug

transport system,

permease component

PF04123

2916_6

Tripartite tricarboxylate transporter (TTT) class transporter

PF04165

594

19512_2

Tripartite tricarboxylate transporter (TTT) class

transporter

PF04165

7776_3

QueT transporter,

involved in queuosine

biosynthetic intermediate uptake

PF06177

1225

1576_4

Uncharacterized

membrane protein, a

putative transporter

component

PF06197

292

1511_3

Uncharacterized

membrane protein, a

putative transporter

component

PF06197

982

uncharacterized transport

system, fused permease

component

PF06808

ECF-type riboflavin

transporter

PF0715

PF07155

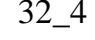

13621_2

ECF-type riboflavin

transporter

PF07155

6319_2

transporter

PF07155

412

major facilitator

superfamily MFS_1

PF07690

10_13

major facilitator

superfamily MFS_1

PF07690

150_4

major facilitator

superfamily MFS_1

PF07690

16_6

major facilitator

superfamily MFS_1

PF07690

$18 \_7$

PF07690 94_2 
Thiamine-precursor transporter protein

ABC-type phosphate

ThiW

PstS

periplasmic component

ABC-type

dipeptide/oligopeptide/nic

kel transport system,

permease component

ABC-type

dipeptide/oligopeptide/nic

kel transport system,

permease component

ABC-type

dipeptide/oligopeptide/nic

kel transport system,

permease component

ABC-type

nitrate/sulfonate/bicarbon

ate transport system,

DppC

DppC

DppC

PF12911,

PF00528

204_3

1143

16996_2

PF12911,

PF00528

529

7812_1

PF12911,

PF00528

975

PF13458 periplasmic component

TRAP-type

uncharacterized transport

system, periplasmic

component

ABC-type $\mathrm{Co} 2+$ transport

system, permease

component

CbiM

TauA

Imp

PF12974

684

ABC-type $\mathrm{Mn} / \mathrm{Zn}$

transport system, ATPase

component

ABC-type branched-chain amino acid transport

system, periplasmic

component

ABC-type branched-chain amino acid transport

system, periplasmic

component

ABC-type branched-chain

amino acid transport

system, periplasmic

component

LivK

LivK

PF13190

4_22

1158

4356_2

ZnuC

PF13304

724

LivK

0_51

1305

1846_4

ABC-type branched-chain amino acid transport

system, periplasmic

component

LivK

PotE

PotE

PotE

PotE

PotE
PF13458

27_12

839

2426_6

PF13458

7_22

87

3560_6

PF13458

PF13520

PF13520

PF13520

PF13520
80_8

$18 \_4$

46_2

69_3

86_1

86_2
PF13520

6514_1
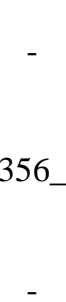

Amino acid transporter
PotE 


\begin{tabular}{|c|c|c|c|c|c|}
\hline $\begin{array}{l}\text { Amino acid transporter } \\
\text { ABC-type molybdate }\end{array}$ & PotE & PF13520 & $86 \_3$ & 845 & - \\
\hline $\begin{array}{l}\text { transport system, } \\
\text { periplasmic component } \\
\text { ABC-type sulfate }\end{array}$ & ModA & PF13531 & 208_2 & 920 & 12421_3 \\
\hline $\begin{array}{l}\text { transport system, } \\
\text { permease component } \\
\text { ABC-type phosphate }\end{array}$ & CysU & $\begin{array}{l}\operatorname{arCOG} 001 \\
64\end{array}$ & $208 \_3$ & 919 & $1742 \_5$ \\
\hline $\begin{array}{l}\text { transport system, } \\
\text { permease component } \\
\text { ABC-type phosphate }\end{array}$ & PstC & $\begin{array}{l}\operatorname{arCOG} 001 \\
67\end{array}$ & 38_2 & 964 & 1514_6 \\
\hline $\begin{array}{l}\text { transport system, } \\
\text { permease component } \\
\text { ABC-type } \\
\text { nitrate/sulfonate/bicarbon }\end{array}$ & PstA & $\begin{array}{l}\operatorname{arCOG} 001 \\
68\end{array}$ & 38_3 & 963 & $1514 \_7$ \\
\hline $\begin{array}{l}\text { ate transport system, } \\
\text { permease component } \\
\text { ABC-type molybdate }\end{array}$ & TauC & $\begin{array}{l}\operatorname{arCOG001} \\
69\end{array}$ & - & 504 & - \\
\hline $\begin{array}{l}\text { transport system, } \\
\text { periplasmic component } \\
\text { ABC-type polar amino }\end{array}$ & ModA & $\begin{array}{c}\operatorname{arCOG002} \\
19\end{array}$ & - & - & 1742_6 \\
\hline $\begin{array}{l}\text { ABC-type polar amino } \\
\text { acid transport system, } \\
\text { ATPase component } \\
\text { ABC-type multidrug }\end{array}$ & $\mathrm{GlnQ}$ & $\begin{array}{l}\operatorname{arCOG} 009 \\
23\end{array}$ & 17_16 & 303 & $2142 \_3$ \\
\hline $\begin{array}{l}\text { transport system, } \\
\text { permease component } \\
\text { Energy-coupling factor } \\
\text { transporter }\end{array}$ & - & $\begin{array}{l}\operatorname{arCOG} 014 \\
65\end{array}$ & & 672 & 4837_2 \\
\hline $\begin{array}{l}\text { transmembrane protein } \\
\text { EcfT } \\
\text { Energy-coupling factor } \\
\text { transporter }\end{array}$ & EcfT & $\begin{array}{l}\operatorname{arCOG} 022 \\
50\end{array}$ & 40_10 & 1444 & $13621 \_3$ \\
\hline $\begin{array}{l}\text { transmembrane protein } \\
\text { EcfT } \\
\text { Energy-coupling factor } \\
\text { transporter }\end{array}$ & EcfT & $\begin{array}{l}\operatorname{arCOG} 022 \\
50\end{array}$ & $65 \_8$ & 298 & $7238 \_2$ \\
\hline $\begin{array}{l}\text { transmembrane protein } \\
\text { EcfT } \\
\text { Energy-coupling factor } \\
\text { transporter }\end{array}$ & EcfT & $\begin{array}{l}\operatorname{arCOG} 022 \\
50\end{array}$ & - & 349 & 7336_3 \\
\hline transmembrane protein & & $\operatorname{arCOG022}$ & & & \\
\hline EcfT & EcfT & 50 & - & 410 & - \\
\hline $\begin{array}{l}\mathrm{Mn} 2+\text { and } \mathrm{Fe} 2+ \\
\text { transporter of the }\end{array}$ & & $\operatorname{arCOG041}$ & & & \\
\hline $\begin{array}{l}\text { NRAMP family } \\
\text { Mn2+ and Fe2+ } \\
\text { transporter of the }\end{array}$ & MntH & $\begin{array}{c}18 \\
\operatorname{arCOG} 041\end{array}$ & - & 959 & 5150_6 \\
\hline $\begin{array}{l}\text { NRAMP family } \\
\text { ABC-2 family transporter }\end{array}$ & $\mathrm{MntH}$ & $\begin{array}{c}18 \\
\operatorname{arCOG} 081\end{array}$ & - & - & $6462 \_3$ \\
\hline protein & - & 48 & - & 727 & - \\
\hline
\end{tabular}

*Annotations from NCBI, Accession number: $\mathrm{Ca}$. K. cryptofilum (CP000968) 
Appendix B: Sulfide sample details from the Eastern Lau Spreading Center
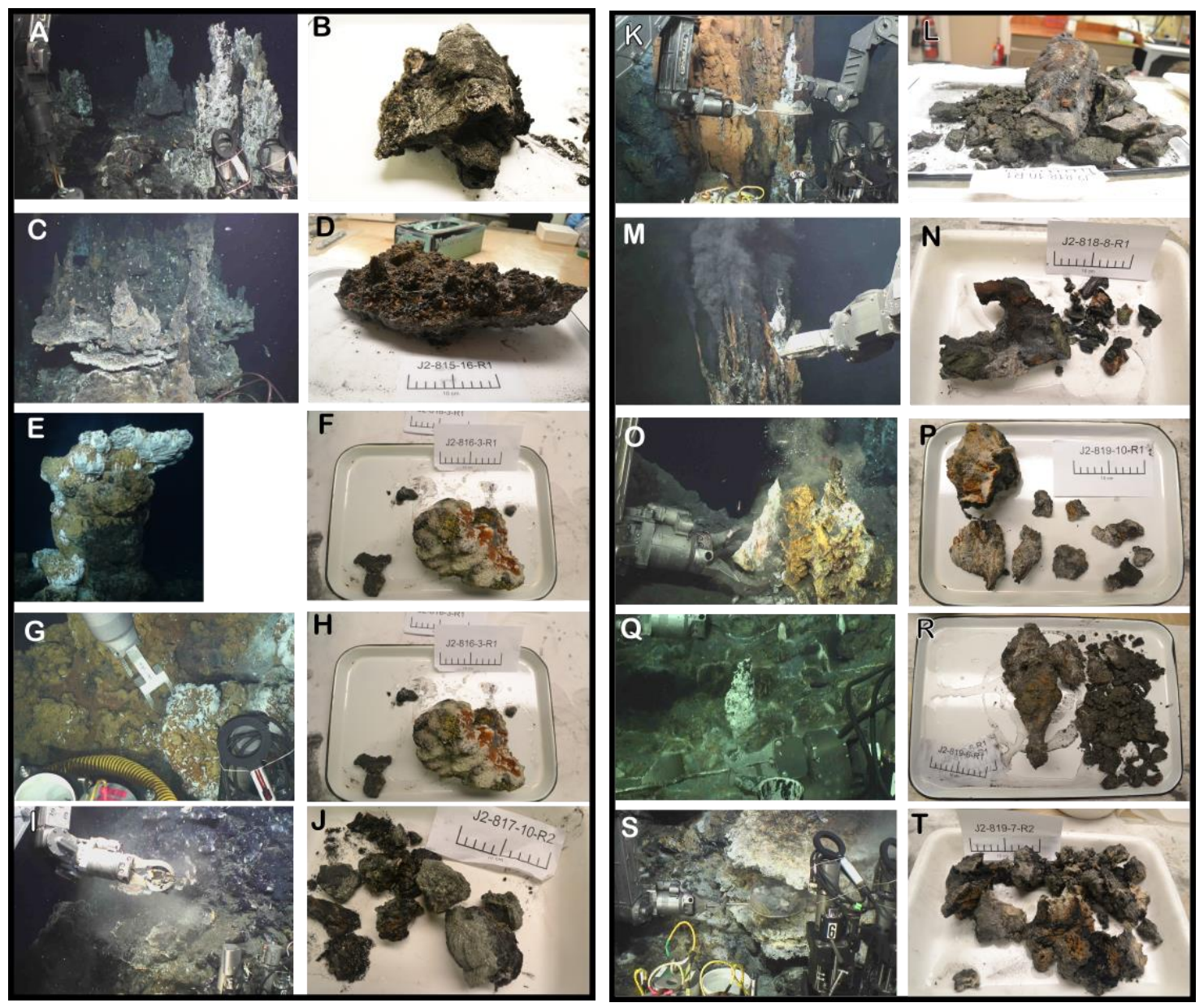

Figure 19. Photographs of samples used for the metagenomic study. Chimney sample ABE-1 on the seafloor (A) and onboard (B). Flange sample ABE-2 on the seafloor (C) and onboard (D). Flange sample Mariner-1 and Mariner-2 on the seafloor, with a pulled back view $(\mathbf{E})$ and up close $(\mathbf{G})$. Mariner-1 and Mariner-2 $(\mathbf{F}, \mathbf{H})$ are from the same rock. Mariner-1 (F) was subsampled from the white biofilm and Mariner-2 (H) was subsampled from the orange biofilm. Flange sample Vai Lili-2 on the seafloor (I) and onboard (J). Chimney sample Mariner-10 on the seafloor (K) and onboard (L). Chimney sample Mariner-17 on the seafloor (M) and onboard (N). Chimney sample Tui Malila-2 on the seafloor (O) and onboard (P). Chimney sample Tui Malila-10 on the seafloor $(\mathbf{Q})$ and onboard $(\mathbf{R})$. Flange sample Tui Malila-11 on the seafloor $(\mathbf{S})$ and onboard (T). 
Table 12. Collection summary of sulfides used for the metagenomes.

\begin{tabular}{lccccc}
\hline Sample & ID & Sub-Type & Date Collected & Latitude (S) & Longitude (W) \\
\hline J2-815-16-R1 & ABE-3 & Flange & $4 / 27 / 2015$ & 2045.954715 & 17611.594939 \\
J2-815-1-R1 & ABE-1 & Chimney & $4 / 27 / 2015$ & 2045.661689 & 17611.458083 \\
J2-816-3-R1 & Mariner-1 & Flange & $4 / 29 / 2015$ & 2010.810572 & 17636.069006 \\
& Mariner-2 & & & & \\
J2-817-10-R2 & Vai Lili-2 & Flange & $4 / 30 / 2015$ & 2212.942024 & 17636.516612 \\
J2-818-10-R1 & Mariner-10 & Chimney & $5 / 8 / 2015$ & 2210.820502 & 17636.100164 \\
J2-818-8-R1 & Mariner-17 & Chimney & $5 / 8 / 2015$ & 2210.806366 & 17636.090282 \\
J2-819-10-R1 & Tui Malila-2 & Chimney & $5 / 10 / 2015$ & 2159.433203 & 17634.152799 \\
J2-819-6-R1 & Tui Malila-10 & Chimney & $5 / 9 / 2015$ & 2159.265642 & 17634.079022 \\
J2-819-7-R2 & Tui Malila-11 & Flange & $5 / 9 / 2015$ & 2159.363164 & 17634.093517 \\
\hline
\end{tabular}

